\title{
An Efficient Flamelet-based Combustion Model for Compressible Flows
}

\author{
Amirreza Saghafian ${ }^{\mathrm{a}, *}$, Vincent E. Terrapon ${ }^{\mathrm{b}}$, Heinz Pitsch $^{\mathrm{c}}$ \\ ${ }^{a}$ Department of Mechanical Engineering, Stanford University, USA \\ ${ }^{b}$ Aerospace and Mechanical Engineering Department, Université de Liège, Belgium \\ ${ }^{c}$ Institute for Combustion Technology, RWTH Aachen University, Germany
}

\begin{abstract}
A combustion model based on a flamelet/progress variable approach for highspeed flows is introduced. In the proposed formulation, the temperature is computed from the transported total energy and tabulated species mass fractions. Only three additional scalar equations need to be solved for the combustion model. Additionally, a flamelet library is used that is computed in a pre-processing step. This approach is very efficient and allows for the use of complex chemical mechanisms. An approximation is also introduced to eliminate costly iterative steps during the temperature calculation. To better account for compressibility effects, the chemical source term of the progress variable is rescaled with the density and temperature. The compressibility corrections are analyzed in an a priori study. The model is also tested in both Reynolds-averaged Navier-Stokes (RANS) and large-eddy simulation (LES) computations of a hydrogen jet in a supersonic transverse flow. Comparison with experimental measurements shows good agreement, partic-
\end{abstract}

\footnotetext{
*Corresponding author

Email address: amirreza@stanford.edu (Amirreza Saghafian)
} 
ularly for the LES case. It is found that the disagreement between RANS results and experimental data is mostly due to the mixing model deficiencies and the presumed probability density functions used in the RANS formulation. A sensitivity study of the proposed model shows the importance of the compressibility corrections especially for the source term of the progress variable.

Keywords: Turbulent Combustion, Compressible Flow, Supersonic

Combustion, Scramjet, Flamelet

\section{Introduction}

As part of the renewed interest in high-speed flight, a need was identified for the development of hypersonic air-breathing propulsion systems using ambient air as oxidizer. These systems have long been recognized as the most well-suited for hypersonic propulsion. Although a traditional ramjet is most appropriate in the supersonic regime (Mach 3 to 5), hypersonic speeds (Mach 6 to 15) can be reached only with the use of a scramjet, where combustion takes place at supersonic speeds. Because of the high speed in scramjet combustors, the flow has a very short residence time inside the engine, during which air and fuel must mix on a molecular level, and chemical reactions have to be completed. Although some ground and flight experiments have successfully demonstrated the feasibility of supersonic combustion [1-4], experimental testing requires a large investment and presents numerous difficulties. Computational tools are thus a key element toward the development of an efficient, high-performance scramjet engine, and because mixing and heat release are at the heart of a scramjet operation, the development and 
use of accurate combustion models for supersonic combustion are critical.

The vast majority of computational modeling work in supersonic turbulent combustion so far has relied on simplified/reduced chemical mechanisms and the explicit transport of the involved species [5]. Such approaches require the closure for the filtered (or ensemble averaged) chemical source term in the species transport equations. This can be achieved, for example, with simpler but low-accuracy models such as the direct use of the Arrhenius law with the mean quantities $[6,7]$, which neglects closure, the Eddy Dissipation Concept model [8], or with closure based on assumed [9, 10] or transported [11-14] probability density functions (PDF). Also, the Linear Eddy model (LEM) $[15,16]$ has been applied for this case. However, due to the strong non-linearity of the source term and the wide range of time scales associated with the chemistry, reactive transport equations are very stiff and difficult to solve. Moreover, due to very short residence times in high speed flows, flame stabilization mechanisms are often governed by auto-ignition. It is critical to model accurately such ignition and extinction phenomena in order to predict the stability of scramjet combustion. Therefore, an approach based on detailed chemical kinetics, which can predict flame stabilization, is required. While a model transporting all involved species can easily be extended to more detailed chemical mechanisms, it quickly becomes computationally intractable, especially when complex fuels must be considered.

An alternative approach is based on the flamelet concept $[17,18]$, which assumes that the chemical time scales are shorter than the turbulent time scales so that the flame can be approximated as an ensemble of laminar flamelets. The so-called steady flamelet approach allows the computation 
of the chemistry to be performed independently of the flow simulation and stored in tabulated form as a function of a limited number of scalars. During the actual simulation, the quantities of interest are read and interpolated, thus, dramatically decreasing the computational cost and allowing the use of complex chemical mechanisms. In the low Mach number flamelet implementation, the temperature and the species mass fraction are assumed to depend only on two scalars, traditionally the mixture fraction and its dissipation rate. Chemical tables are then constructed assuming constant background pressure. This formulation can also be extended to better reproduce the unsteady character of combustion by replacing the scalar dissipation rate with a progress variable $[19,20]$.

The typical implementation of the flamelet model is based on a low Mach number assumption, explaining the still very limited number of studies of high-speed flows using this approach [21-23]. Kumar and Tamaru [23] used a laminar flamelet model to simulate a compressible ram combustor, where the temperature was interpolated from a flamelet library based on the mixture fraction and scalar dissipation rate. Oevermann [22] extended this model by computing the temperature from the internal energy and the species mass fractions, where the energy is determined from the solution of a transport equation, and the species mass fractions were interpolated from a flamelet table, based on the same parameters and without introducing any corrections to the low Mach number flamelet model. Berglund and Fureby [21] used a one-equation (mixture fraction) and a two-equation (mixture fraction and progress variable) flamelet model in conjunction with a two-step reaction mechanism. This combustion model is also based on the low Mach number 
flamelet approach without any compressibility correction. Vicquelin et al. [24] also developed a formalism to couple chemistry tabulation to solvers for mildly compressible flows. They computed temperature from the internal energy using a simple expansion, whereas no extension has been used for other quantities. This approach is reasonable for low Mach number compressible flows, but cannot accurately describe the high Mach number regime.

As shown in this study, the low Mach number assumption does not hold anymore at supersonic speed, where strong compressibility effects and viscous heating start to play an important role. For instance, the source term of the progress variable is extremely sensitive to the variations in temperature and pressure, and appropriate modifications should be implemented. Therefore, without appropriate compressibility corrections, a combustion model based on the low Mach number assumptions is not plausible for compressible flows, especially at high Mach numbers, where flows can admit shock waves and expansion fans.

We introduce here two different formulations of a compressible flamelet/ progress-variable approach, where temperature is not given by a chemistry table, but computed from the total energy and the species mass fractions. An analytical relationship is derived to eliminate costly iterative steps during the temperature calculation. In addition, the source term for the progress variable is rescaled by the mixture temperature and density, thus better accounting for compressibility effects on chemistry. Compressibility corrections have been devised also for the mixture properties, which are sensitive to the compressible variations of temperature and pressure. The model is tested in both LES and RANS computations for a reacting hydrogen jet in a supersonic 
transverse flow.

In the next section, the equations for the transported quantities are summarized. Section 3 describes the new combustion model and its implementation. The compressibility corrections are validated in section 4 and applied to the case of a jet in a supersonic cross-flow in section 5. The description of the flow configuration is given in section 5.1, the numerical implementation in section 5.2, and results are presented in section 5.3. A sensitivity analysis of the proposed model to the model assumptions is provided in section 6 . Finally, we conclude with suggestions for future improvements.

\section{Governing equations}

In the following, we will discuss modeling of supersonic combustion both in the context of RANS and LES. The governing equations have a similar form, and, therefore, will be presented only once. The averaging operators appearing in these equations refer to an ensemble average in RANS, and to a spatial filter in LES. The relevant operators ${ }^{-}$and $\tilde{\cdot}$ denote direct and Favre averaging or filtering, respectively.

Transport equations for the Favre averaged or filtered variables $\bar{\rho}, \bar{\rho} \widetilde{u}_{i}, \bar{\rho} \widetilde{E}$, $\bar{\rho} \widetilde{Z}, \bar{\rho} \widetilde{Z^{\prime \prime 2}}$, and $\bar{\rho} \widetilde{C}$ are solved in conservative form, where $\bar{\rho}$ is the density, $\widetilde{u}_{i}$ the components of the velocity vector, $\widetilde{E}$ the total energy, including the chemical energy, $\widetilde{Z}$ the mixture fraction, $\widetilde{Z^{\prime \prime 2}}$ the variance of the mixture

fraction, and $\widetilde{C}$ a progress variable. Their respective transport equations are

$$
\frac{\partial \bar{\rho}}{\partial t}+\frac{\partial \bar{\rho} \widetilde{u}_{j}}{\partial x_{j}}=0
$$




$$
\begin{gathered}
\frac{\partial \bar{\rho} \widetilde{u}_{i}}{\partial t}+\frac{\partial \bar{\rho} \widetilde{u}_{i} \widetilde{u}_{j}}{\partial x_{j}}=-\frac{\partial \bar{p}}{\partial x_{i}}+\frac{\partial \bar{\tau}_{j i}}{\partial x_{j}}+\frac{\partial \bar{\tau}_{j i}^{R}}{\partial x_{j}} \\
\frac{\partial \bar{\rho} \tilde{E}}{\partial t}+\frac{\partial \bar{\rho} \tilde{E} \widetilde{u}_{j}}{\partial x_{j}}=-\frac{\partial \bar{p} \widetilde{u}_{j}}{\partial x_{j}}+\frac{\partial \bar{\tau}_{j i} \widetilde{u}_{i}}{\partial x_{j}}-\frac{\partial \bar{q}_{j}}{\partial x_{j}}+\frac{\partial \bar{\pi}_{j}^{R}}{\partial x_{j}}+\widetilde{u}_{i} \frac{\partial \bar{\tau}_{j i}^{R}}{\partial x_{j}} \\
\frac{\partial \bar{\rho} \widetilde{Z}}{\partial t}+\frac{\partial \tilde{\rho}_{j} \widetilde{Z}}{\partial x_{j}}=\frac{\partial}{\partial x_{j}}\left(\bar{\rho} D_{Z} \frac{\partial \widetilde{Z}}{\partial x_{j}}\right)+\frac{\partial \bar{\tau}_{j}^{Z}}{\partial x_{j}} \\
\frac{\partial \bar{\rho} Z^{\prime \prime 2}}{\partial t}+\frac{\partial \bar{\rho} \widetilde{u}_{j} \widetilde{Z^{\prime \prime 2}}}{\partial x_{j}}=\frac{\partial}{\partial x_{j}}\left(\bar{\rho} D_{Z} \frac{\partial \widetilde{Z}^{\prime \prime 2}}{\partial x_{j}}\right)+\frac{\partial \bar{\tau}_{j}^{Z^{\prime \prime 2}}}{\partial x_{j}}+2 \frac{\mu_{t}}{\mathrm{Sc}_{t}} \frac{\partial \widetilde{Z}_{j}}{\partial x_{j}} \frac{\partial x_{j}}{\bar{Z}}-\widetilde{\chi}^{R}, \\
\frac{\partial \bar{\rho} \widetilde{C}}{\partial t}+\frac{\partial \bar{\rho} \widetilde{u}_{j} \widetilde{C}}{\partial x_{j}}=\frac{\partial}{\partial x_{j}}\left(\bar{\rho} D_{C} \frac{\partial \widetilde{C}}{\partial x_{j}}\right)+\frac{\partial \bar{\tau}_{j}^{C}}{\partial x_{j}}+\overline{\dot{\omega}}_{C},
\end{gathered}
$$

where

$$
\begin{aligned}
& \bar{\tau}_{i j}^{R}=\bar{\rho} \widetilde{u}_{i} \widetilde{u}_{j}-\bar{\rho} \widetilde{u_{i} u_{j}} \\
& q_{i}=-\frac{\lambda}{c_{p}} \frac{\partial h}{\partial x_{i}}+\sum_{\alpha=1}^{N}\left[\rho Y_{\alpha} V_{\alpha i}-\frac{\lambda}{c_{p}} \frac{\partial Y_{\alpha}}{\partial x_{i}}\right] h_{\alpha}, \\
& \bar{\pi}_{i}^{R}=\bar{\rho} \tilde{e} \widetilde{u}_{j}-\bar{\rho} \widetilde{e u_{j}}, \\
& \bar{\tau}_{i}^{Z}=\bar{\rho} \widetilde{u}_{i} \widetilde{Z}-\widetilde{\rho} \widetilde{u_{i} Z} \\
& \bar{\tau}_{i}^{Z^{\prime 2}}=\bar{\rho} \widetilde{u}_{i} \widetilde{Z^{\prime \prime 2}}-\bar{\rho} \widetilde{u_{i} Z^{\prime \prime 2}} \\
& \bar{\tau}_{i}^{C}=\bar{\rho} \widetilde{u}_{i} \widetilde{C}-\widetilde{\rho} \widetilde{u_{i} C}
\end{aligned}
$$

$\mu$ and $\mu_{t}$ are the laminar and turbulent viscosity, $k$ is the turbulent kinetic energy, $\lambda$ the thermal diffusivity, $c_{p}$ the specific heat capacity at constant pressure, $\operatorname{Pr}_{t}$ a turbulent Prandtl number, $h$ the enthalpy including the sensible 
and chemical contributions, $Y_{k}$ the species mass fractions, $\sigma_{k}$ the turbulent kinetic energy Schmidt number, $\tau_{i j}$ the viscous stress tensor, $D_{Z}$ the diffusion coefficient for the mixture fraction, $D_{C}$ the diffusion coefficient for the progress variable, $\mathrm{Sc}_{t}$ a turbulent Schmidt number, $\chi$ the scalar dissipation rate, and $\dot{\omega}_{C}$ the source term for the progress variable. The molecular properties $\mu, \lambda, c_{p}$ are computed using mixing rules [25] to account for changes in composition and temperature. The turbulent viscosity $\mu_{t}$ and kinetic energy $k$ are computed in the RANS context using the shear stress transport (SST) model [26] or based on the dynamic subgrid-scale model [27] in the LES context. For more information about the governing equations and unclosed terms refer to Saghafian [28] and Ihme [29].

Note that the summation on the right-hand side of Eq. (8) vanishes under the unity Lewis number assumption (using Fick's law) so that the species mass fraction gradients do not need to be explicitly computed. Moreover, the sub-grid terms are neglected in this equation.

The mixture composition depends directly on $\widetilde{Z}, \widetilde{Z^{\prime \prime 2}}$, and $\widetilde{C}$. In order to close this system, the pressure and the temperature need to be determined. This is achieved, as explained in the following section, by using an equation of state and the energy of the mixture. For hydrogen chemistry, the progress variable is defined as the mass fraction of $\mathrm{H}_{2} \mathrm{O}$.

\section{Combustion model}

The low Mach number flamelet/progress variable approach is summarized in section 3.1. Two extensions of this model for compressible flows are then introduced in sections 3.2 and 3.3. In all cases, the models are based on the 
flamelet/progress variable approach, in which the chemistry is pre-computed and tabulated as a series of laminar flamelet solutions for a given set of boundary conditions and background pressure.

\subsection{Low Mach number flamelet/progress variable approach}

The low Mach number flamelet/progress variable (FPV) approach [17$20,29,30]$ is based on the steady flamelet equations [17,31], which are derived by using a transformation of the species and energy transport equations from the physical space to a mixture fraction based space. The steady flamelet equations are

$$
-\rho \frac{\chi_{Z}}{2} \frac{\partial^{2} Y_{\alpha}}{\partial Z^{2}}=\dot{\omega}_{\alpha} \quad \alpha=1,2, \ldots, N .
$$

Although these are steady equations, the solutions capture much of the dynamics of flames, for instance extinction at high mixing (scalar dissipation) rates. This behavior can be visualized by considering the stoichiometric temperature as function of the Damköhler number (inverse of scalar dissipation rate), which results in a S-shaped curve, resulting from the strong non-linearities of the interaction of mixing and chemistry [32]. The scalar

dissipation rate, $\chi_{Z}=2 D_{Z}|\nabla Z|^{2}$ appearing as a parameter in Eq. (13), describes the local effect of molecular mixing on the chemistry, thus displaying the interplay between turbulence and combustion.

In order to solve Eq. (13), the scalar dissipation rate, a set of boundary conditions (including temperature and mixture composition) at the fuel and oxidizer sides, and a background pressure should be specified. Different models for the scalar dissipation rate can be devised resembling the local 
structure of a counterflow diffusion flame [17] or a semi-infinite mixing layer [33].

A reaction progress parameter, $\Lambda$, is introduced based on the progress variable $[19,29]$ to uniquely identify an associated flamelet. For each flamelet, $\Lambda$ is defined as the value of the progress variable $C$ at $Z=Z_{s t}$, where $Z_{s t}$ is the stoichiometric value of mixture fraction. The reaction progress parameter then is independent of mixture fraction and replaces the scalar dissipation rate in the chemistry parametrization used in the flamelet model. In a CFD simulation, the equations for mixture fraction $Z$ and the progress variable $C$ are solved. For a given state, defined by $Z$ and $C$, the reaction progress parameter, $\Lambda$, and hence the corresponding flamelet solution, can be determined in a chemical table. This inversion and associated assumptions and difficulties are discussed in Ihme and Pitsch [32]. Assumed PDFs are introduced to account for the turbulence/chemistry interaction (e.g. to compute the filtered source term in Eq. (6)). Typically, a $\beta$-function is assumed for the mixture fraction $Z$ and a $\delta$-function for the PDF of the progress variable $C$. Therefore, the flamelet library is parametrized by the mean mixture fraction $\widetilde{Z}$, the variance of the mixture fraction $\widetilde{Z^{\prime \prime 2}}$, and the mean progress variable $\widetilde{C}$. The temperature is usually looked up from the flamelet library.

\subsection{Compressible extended flamelet table approach}

A fluid region in a compressible flow can experience strong variations in temperature and pressure due to viscous heating, shock waves, or strong expansions. To account for these compressibility effects, two additional degrees of freedom could be added to the manifold representing the thermochemical state of the flamelets. Typically, pressure and an energy-related quantity 
(e.g. internal energy or enthalpy) could be considered as additional dimensions to the flamelet library. The use of a flamelet library computed under the low Mach assumption, but in an state space extended by pressure and energy should be called here the compressible extended flamelet table (CEFT) approach [28]. Although the dimensionality of the problem increases for such an approach, it still remains a low-dimensional representation of combustion.

In this compressible extended flamelet table (CEFT) approach, the Sshaped curve solutions to the flamelet equations typically used in the FPV

model is expanded to an S-shaped hypersurface. This hypersurface is defined by all the flamelet solutions of Eq. (13) considering all possible variations of the boundary temperatures and the background pressure.

However, the memory requirement of a five-dimensional table is often prohibitive, especially for hydrocarbon fuels. This motivates the development of a simplified version of this model that limits the dimensionality of the chemistry table, as explained next.

\subsection{Compressible flamelet/progress variable approach}

The main idea of the compressible flamelet/progress variable (CFPV) approach is to reduce the dimensionality of the chemistry table by representing the S-shaped hypersurface as a perturbation around a nominal low Mach number flamelet solution [28]. In other words, the additional dimensions introduced in the CEFT approach above are approximated by an analytical surface. The exact analytical form of this expression can vary depending on the quantity considered, but as an example, a quantity of interest $\phi$ that 
needs to be tabulated can be written as

$$
\phi\left(\widetilde{Z}, \widetilde{Z^{\prime \prime 2}}, \widetilde{C}, \bar{p}, \widetilde{e}\right)=\phi_{0}\left(\widetilde{Z}, \widetilde{Z^{\prime \prime 2}}, \widetilde{C} ; \overline{p_{0}}, \widetilde{e_{0}}\right)+\Phi\left(\bar{p}, \widetilde{e} ; \widetilde{Z}, \widetilde{Z^{\prime \prime 2}}, \widetilde{C}\right),
$$

where $e$ represents the internal energy including the chemical energy, and $\phi_{0}$ is the value of $\phi$ obtained at reference conditions corresponding to $p_{0}$ and $e_{0}$. On the other hand, $\Phi$ is an analytical expression that represents the deviation of $\phi$ at the local conditions $p$ and $e$ with respect to the reference conditions. Note that the analytical expression $\Phi$ depends on parameters that are typically functions of $\widetilde{Z}, \widetilde{Z^{\prime \prime 2}}$, and $\widetilde{C}$, and are thus tabulated in the chemistry table, as illustrated below. This leads to a chemistry table with three dimensions, as in the low Mach number FPV method, which is computationally tractable. Such expansions have been proposed by Wang et al. [34], and have been used for low Mach number flows [35-37].

Typical quantities of interest that are not known from the transport equations include species mass fractions, gas constant, temperature, pressure, molecular viscosity, and thermal diffusivity. The remaining part of this section describes the analytical perturbations used for these quantities.

The first assumption is that the species mass fractions do not strongly vary if the deviation in pressure and temperature from reference conditions is not too large. In other words, it is assumed that the species mass fractions are frozen at the reference level:

$$
Y_{\alpha}\left(\widetilde{Z}, \widetilde{Z^{\prime \prime 2}}, \widetilde{C}, \bar{p}, \widetilde{e}\right)=Y_{\alpha, 0}\left(\widetilde{Z}, \widetilde{Z^{\prime \prime 2}}, \widetilde{C} ; \overline{p_{0}}, \widetilde{e_{0}}\right)
$$

This is a rather strong assumption, which is discussed in the next section. A direct consequence is that the gas constant becomes

$$
R\left(\widetilde{Z}, \widetilde{Z^{\prime \prime 2}}, \widetilde{C}, \bar{p}, \widetilde{e}\right)=R_{0}\left(\widetilde{Z}, \widetilde{Z^{\prime \prime 2}}, \widetilde{C} ; \overline{p_{0}}, \widetilde{e_{0}}\right)
$$


and can be directly tabulated.

Given a mixture composition, the temperature can be computed from the total energy. The total energy $\widetilde{E}$ of the $N$ species mixture is defined as the sum of the internal energy, $\widetilde{e}$, the kinetic energy $1 / 2 \widetilde{u}_{j} \widetilde{u}_{j}$, and the turbulent kinetic energy $k$. The internal energy is

$$
\widetilde{e}=\widetilde{h}-\widetilde{R T}=\sum_{\alpha=1}^{N} \widetilde{Y_{\alpha} h_{\alpha}}-\widetilde{R T},
$$

where $R=R_{u} \sum_{\alpha} Y_{\alpha} / W_{\alpha}$ is the gas constant, $R_{u}$ the universal gas constant, $W_{\alpha}$ the molecular weight of species $\alpha$, and $T$ the temperature. The specific enthalpy of species $\alpha$ is computed as

$$
h_{\alpha}(T)=h_{\alpha}^{0}\left(T_{r e f}\right)+\int_{T_{r e f}}^{T} c_{p, \alpha}\left(T^{\prime}\right) d T^{\prime},
$$

using its specific heat capacity $c_{p, \alpha}$ and heat of formation $h_{\alpha}^{0}\left(T_{r e f}\right)$. Due to the wide range of temperature variations caused by compressibility in a high-speed flow, the dependence of the heat capacity on temperature must be considered.

Since the total energy is a non-linear function of the temperature, an iterative method like Newton-Raphson is required to compute the temperature given the energy $\widetilde{E}$ and the mixture composition. In order to eliminate this expensive iterative step, an alternate approach is proposed. For a given mixture, i.e. for fixed $\widetilde{Z}, \widetilde{Z^{\prime \prime 2}}$, and $\widetilde{C}$, the internal energy can be approximated as

$$
\widetilde{e}=\widetilde{e}_{0}+\int_{T_{0}}^{\widetilde{T}} \widetilde{c}_{v}(T) d T \approx \widetilde{e}_{0}+\int_{T_{0}}^{\widetilde{T}} \frac{\widetilde{R}}{\widetilde{\gamma}(T)-1} d T,
$$

where $\gamma$ is the ratio of the specific heats, and the correlations between the fluctuations of the gas constant and the fluctuations of the specific heat ratio 
are neglected. $\widetilde{\gamma}$ can then be expanded about the flamelet solution using a linear expansion in temperature,

$$
\widetilde{\gamma}(T)=\widetilde{\gamma}_{0}+a_{\gamma}\left(\widetilde{T}-T_{0}\right)
$$

The values of $\widetilde{e}_{0}, \widetilde{R}_{0}, \widetilde{\gamma}_{0}, a_{\gamma}$, and $T_{0}$ can be computed during a preprocessing step and tabulated in the flamelet library. This approximation then leads to an analytical relationship between temperature and specific energy

$$
\widetilde{e}=\widetilde{e}_{0}+\frac{\widetilde{R}}{a_{\gamma}} \ln \left(1+\frac{a_{\gamma}\left(\widetilde{T}-T_{0}\right)}{\widetilde{\gamma}_{0}-1}\right),
$$

which can be inverted to yield the temperature as

$$
\widetilde{T}=T_{0}+\frac{\widetilde{\gamma}_{0}-1}{a_{\gamma}}\left(\mathrm{e}^{a_{\gamma}\left(\widetilde{e}-\widetilde{e}_{0}\right) / \widetilde{R}}-1\right) .
$$

The temperature dependencies of the molecular viscosity and thermal diffusivity are described by the following power-law corrections,

$$
\begin{aligned}
& \frac{\widetilde{\mu}}{\widetilde{\mu_{0}}}=\left(\frac{\widetilde{T}}{T_{0}}\right)^{a_{\mu}}, \\
& \frac{\widetilde{\lambda}}{\widetilde{\lambda_{0}}}=\left(\frac{\widetilde{T}}{T_{0}}\right)^{a_{\lambda}},
\end{aligned}
$$

where $a_{\mu}$ and $a_{\lambda}$ are functions of $\widetilde{Z}, \widetilde{Z^{\prime \prime 2}}$, and $\widetilde{C}$, and are stored in the flamelet library along with $T_{0}, \mu_{0}$, and $\lambda_{0}$. These coefficients are computed by perturbing the flamelet solutions and comparing the value of the corresponding variable at the perturbed and reference conditions [28].

In order to completely close the system of equations (1)-(6), the equation of state for an ideal gas is used to compute the pressure

$$
\bar{p}=\widetilde{\rho} \widetilde{R T}
$$


and it is assumed that $\widetilde{R T} \approx \widetilde{R} \widetilde{T}[28]$.

Although mixture properties not depending on temperature (e.g., gas constant) are not very sensitive to the compressibility effects (see section 4), the source term of the progress variable is very sensitive to these perturbations. Therefore, a rescaling for this term is introduced to account for these perturbations due to compressibility. The source term in Eq. (6) is thus rescaled as

$$
\frac{\overline{\dot{\omega}}_{C}}{\overline{\dot{\omega}}_{C_{0}}}=\left(\frac{\bar{\rho}}{\bar{\rho}_{0}}\right)^{a_{\rho}} \exp \left[-T_{a}\left(\frac{1}{\widetilde{T}}-\frac{1}{T_{0}}\right)\right],
$$

where $\overline{\dot{\omega}}_{C_{0}}$ is the tabulated source term computed at a background pressure $p_{0}$. As before, the values $T_{0}, \rho_{0}, a_{\rho}$, and $T_{a}$ are computed in a preprocessing step to describe the dependency of the source term on the mixture temperature and pressure, and are then tabulated as a function of $\widetilde{Z}, \widetilde{Z^{\prime \prime 2}}$, and $\widetilde{C}$. This equation is motivated from the Arrhenius behavior of elementary reactions.

\section{A priori analysis of compressibility effects}

The CFPV model described above relies on different assumptions that are quantified and discussed here. As explained above, the reference condition is controlled by the boundary temperatures and background pressure of the flamelet solutions. These quantities should be chosen such that they represent the conditions of the flow as good as possible. For example, the oxidizer temperature $T_{o x}$ can be set to the free stream temperature and the fuel temperature to the fuel total temperature.

The assumption that the composition is not changed by disturbances of energy and pressure around the reference state is assessed first. Figure 1 
shows the species mass fractions of the major and important minor species for three flamelet solutions computed with different values of the fuel temperature. To be consistent with the flamelet/progress variable approach, the flamelets used in this and the following comparisons are chosen such that they have an equal reaction progress parameter defined as the value of the progress variable at stoichiometric condition. It can be seen that the mixture composition changes little with varying fuel temperature. This can be explained by the fact that the flame is located very close to the oxidizer side, because the stoichiometric mixture fraction is 0.03 for hydrogen. Consequently, larger changes in the mixture composition are observed when the oxidizer temperature is varied, as shown in Fig. 2, but these variations remain small for the major species. Although minor species show a stronger departure from their nominal values, their contributions to the mixture properties are much smaller than those of the major species. For instance, Fig. 3 shows that the gas constant of the mixture does not significantly vary with the oxidizer temperature. Finally, Fig. 4 compares the mixture composition of three flamelet solutions with different values of the background pressure. The disparity in the mass fractions of major species is much smaller than for the minor species, and again these variations do not significantly change those mixture properties (such as the gas constant) that are independent of the mixture temperature.

Note that although this analysis has been illustrated with the example of an arbitrarily chosen flamelet, the same conclusions are obtained for the entire S-shaped curve (i.e., for different values of the scalar dissipation rate $\chi)$. In other words, properties that only depend on the mixture composition 

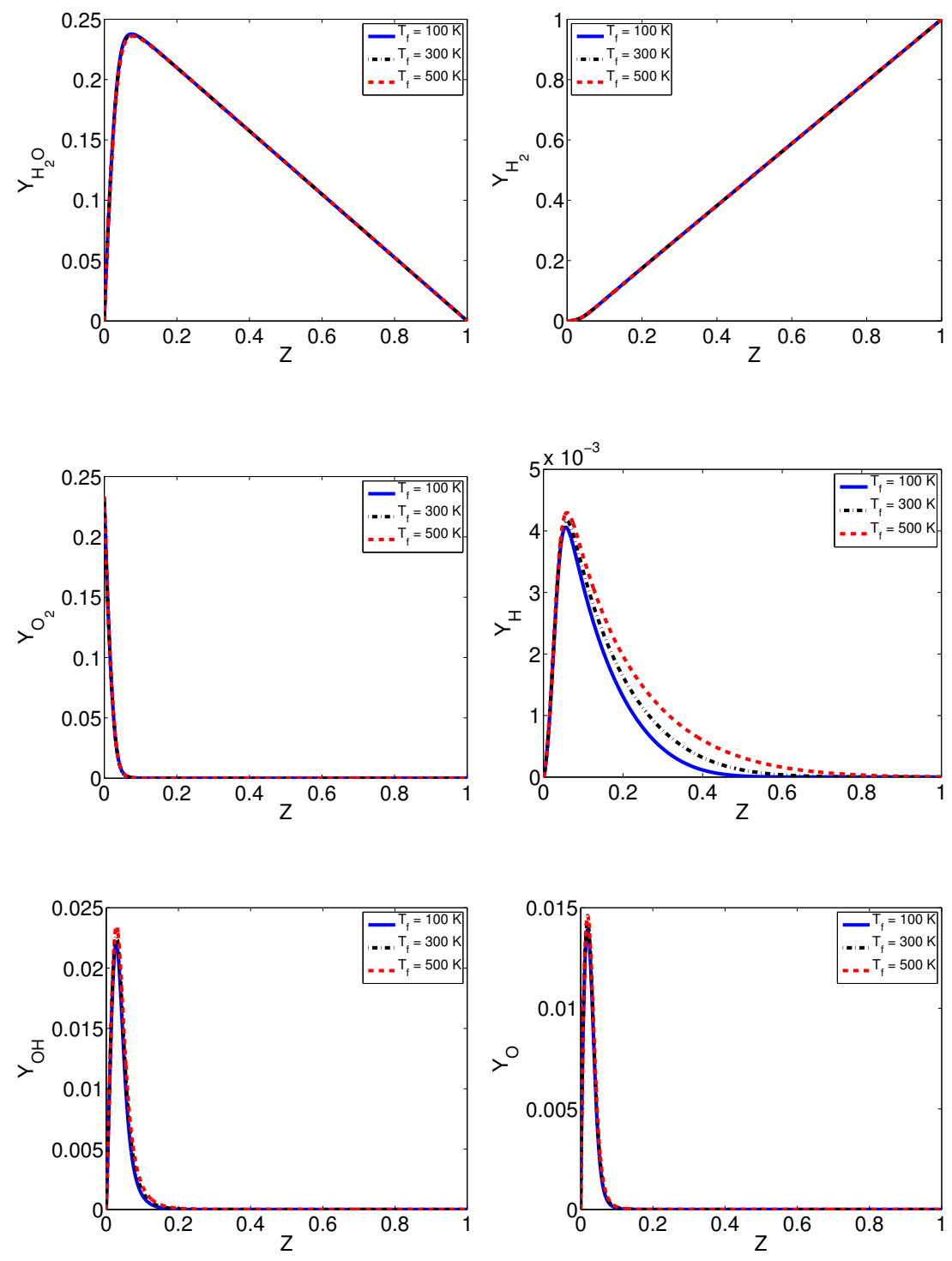

Figure 1: Species mass fractions of the major species $\left(\mathrm{H}_{2} \mathrm{O}, \mathrm{H}_{2}, \mathrm{O}_{2}\right)$ and important minor species $(\mathrm{H}, \mathrm{OH}, \mathrm{O})$ obtained from the solution of the flamelet equations for three values of the fuel temperature, $T_{f}$, fixed oxidizer temperature, $T_{o x}=1550 \mathrm{~K}$, and fixed background pressure, $p=1$ bar. All flamelet solutions have the same value of the reaction progress parameter. 

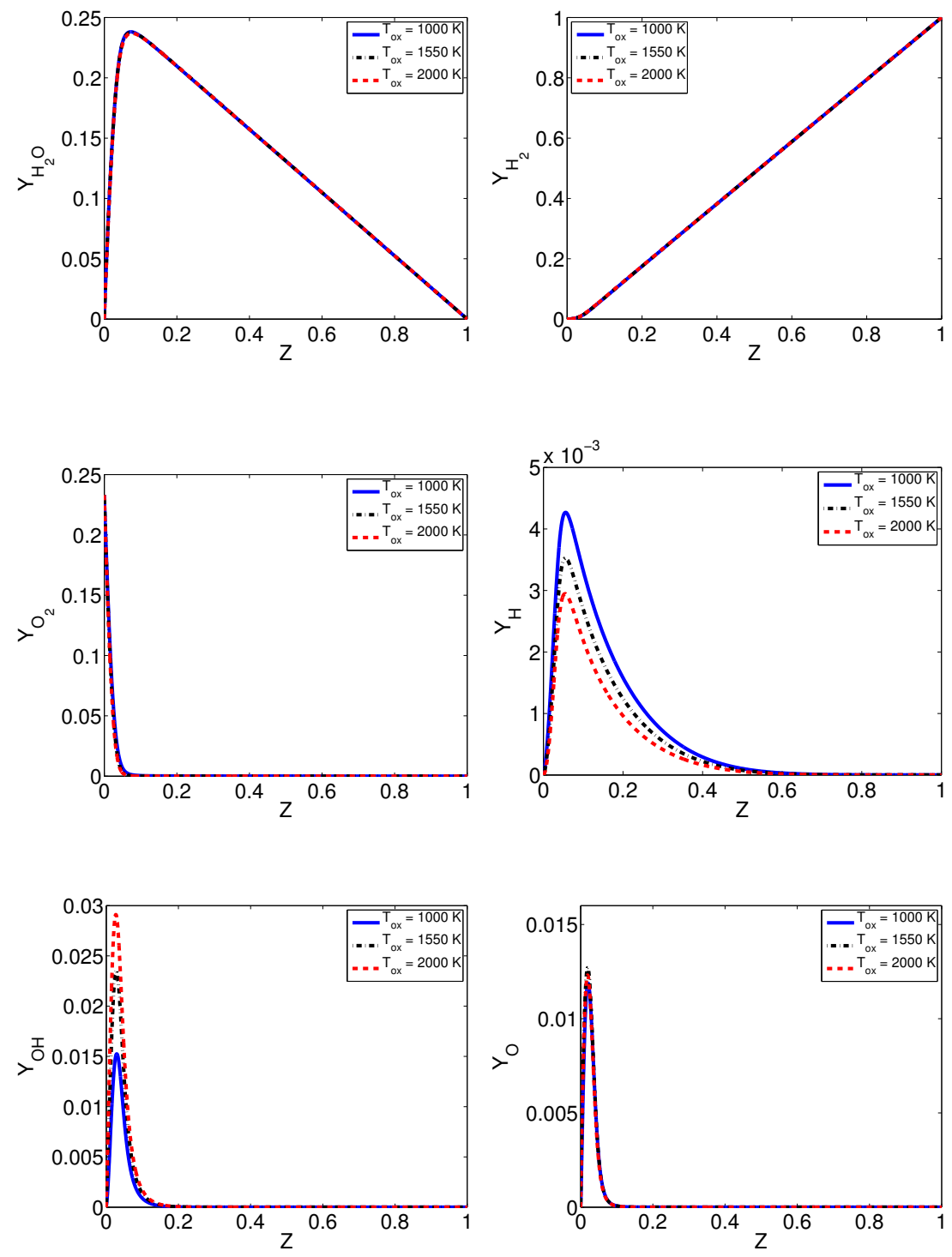

Figure 2: Species mass fraction of the major species $\left(\mathrm{H}_{2} \mathrm{O}, \mathrm{H}_{2}, \mathrm{O}_{2}\right)$ and important minor species $(\mathrm{H}, \mathrm{OH}, \mathrm{O})$ obtained from the solution of the flamelet equations for three values of the oxidizer temperature, $T_{o x}$, a fixed fuel temperature, $T_{f}=300 \mathrm{~K}$, and fixed background pressure $p=1$ bar. All flamelet solutions have the same value of the reaction progress parameter. 


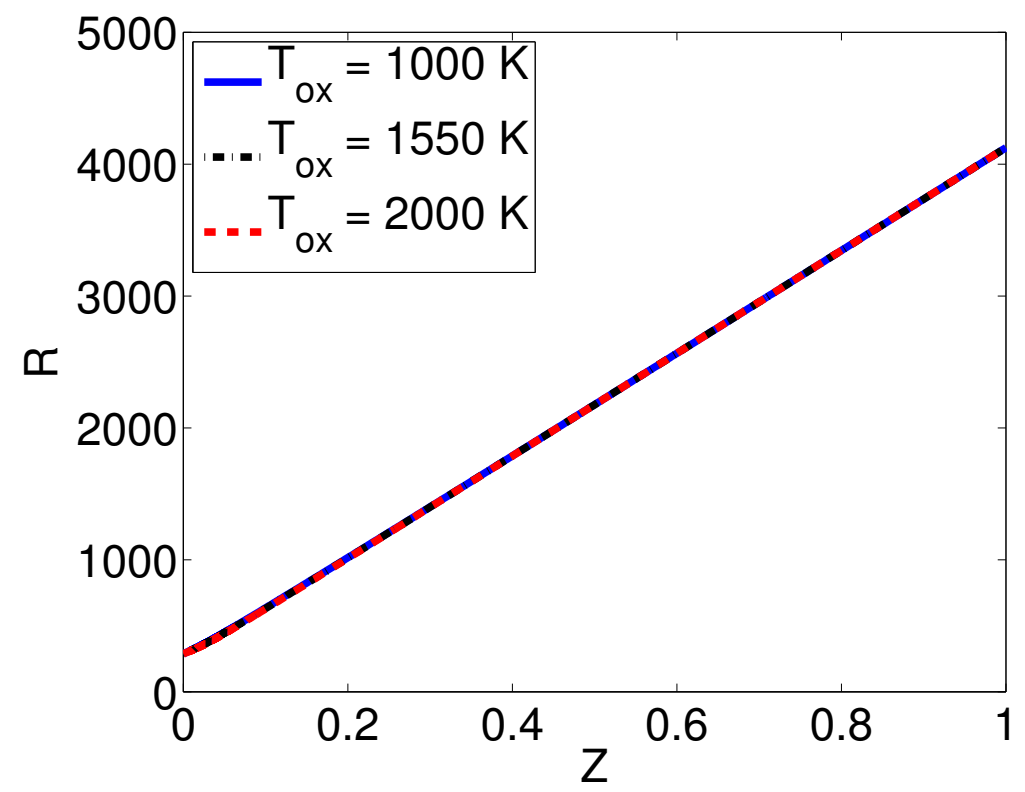

Figure 3: Gas constant, $R$, obtained from the solution of the flamelet equations for three values of the oxidizer temperature, $T_{o x}$, fixed fuel temperature, $T_{f}=300 \mathrm{~K}$, and fixed background pressure $p=1$ bar. All flamelet solutions have the same value of the reaction progress parameter. 



Figure 4: Species mass fractions of the major species $\left(\mathrm{H}_{2} \mathrm{O}, \mathrm{H}_{2}, \mathrm{O}_{2}\right)$ and important minor species $(\mathrm{H}, \mathrm{OH}, \mathrm{O})$ obtained from the solution of the flamelet equations for three values of the background pressure, $p$, fixed fuel temperature, $T_{f}=300 \mathrm{~K}$, and fixed oxidizer temperature, $T_{o x}=1550 \mathrm{~K}$. All flamelet solutions have the same value of the reaction progress parameter. 
can be estimated with relatively good accuracy with the flamelet solutions computed at the reference values. However, the calculation of properties that also depend on the temperature (e.g., specific heat ratio and viscosity) must account for temperature variations.

In order to validate Eqs. (20), (22), and (26), a flamelet library is generated at reference conditions $T_{o x}=1550 \mathrm{~K}, T_{f}=300 \mathrm{~K}$, and $p=1.0$ bar. This table is then used to estimate different properties of a flamelet solution with significantly higher temperature, $T_{o x}=2000 \mathrm{~K}$, in order to test the ability of the perturbation functions introduced above to approximate the S-shaped hypersurface. The temperature is first computed using the internal energy of the perturbed flamelet and the mixture composition from the flamelet library at the reference conditions. Figure 5a shows that computing the temperature from Eq. (22) (red curve) leads to a maximum relative error smaller than $3 \%$. This approach, based on the analytical relation between temperature and internal energy, is then compared to the iterative solution of Eq. (17). The difference between the temperature obtained from Eq. (22) and from solving Eq. (17) iteratively is plotted as a function of the mixture fraction in Fig. 5b. The maximum difference is about two Kelvins, which shows the good accuracy of the approximation (Eq. (22)). Using this approximation improves the computational efficiency of the CFPV method, especially for LES computations where explicit temporal integration is used. Similarly, the error committed using a linear expansion in temperature for the specific heat ratio (Eq. (20)) leads to a maximum error that is less than $0.3 \%$. This is shown in Fig. 6.

The source term of the progress variable is extremely sensitive to per- 


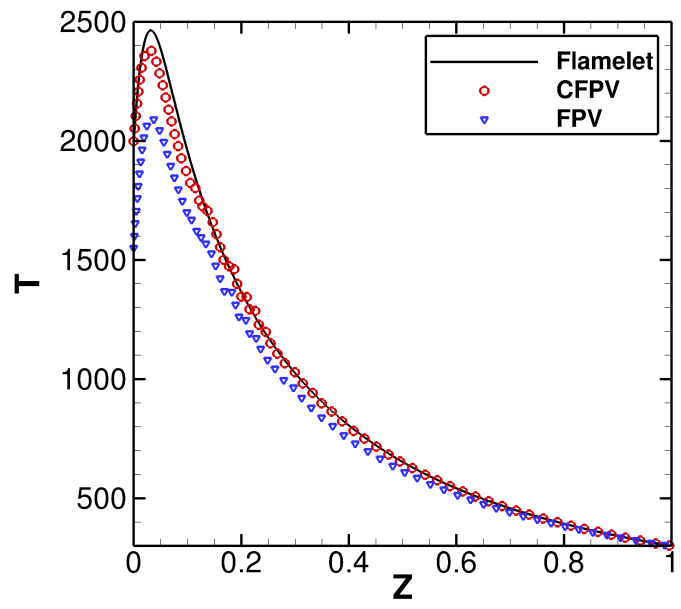

(a) Temperature



(b) Temperature discrepancy

Figure 5: Temperature versus mixture fraction (left), and the difference between the temperature obtained from the analytical expression, Eq. (22), and from solving iteratively Eq. (17) (right). Black line corresponds to flamelet with $T_{o x}=2000 \mathrm{~K}, T_{f}=300 \mathrm{~K}$, and $p=1$ bar. Red circles show CFPV results computed from chemical library at reference conditions, $T_{o x}=1550 \mathrm{~K}$, $T_{f}=300 \mathrm{~K}, p=1.0$ bar. Blue triangles represent temperature corresponding to reference condition. 


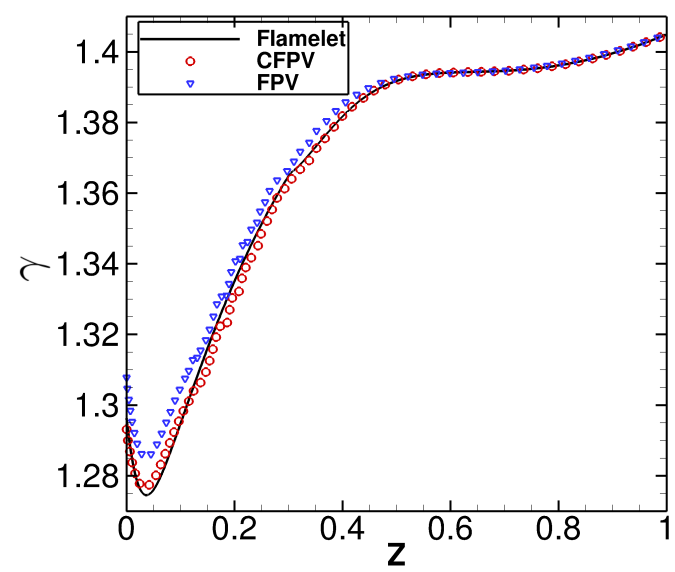

Figure 6: Specific heat ratio as function of mixture fraction. Black line corresponds to flamelet with $T_{o x}=2000 \mathrm{~K}, T_{f}=300 \mathrm{~K}$, and $p=1$ bar. Red circles show CFPV results computed from chemical library at reference conditions, $T_{o x}=1550 \mathrm{~K}, T_{f}=300 \mathrm{~K}, p=1.0 \mathrm{bar}$. Blue triangles represent values at reference condition. 


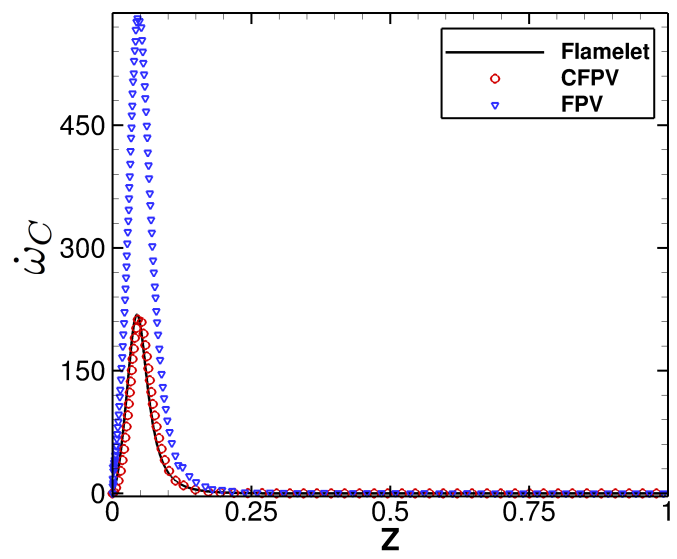

Figure 7: Source term of the progress variable versus mixture fraction. Black line is flamelet with $T_{o x}=2000 \mathrm{~K}, T_{f}=300 \mathrm{~K}$, and $p=1$ bar. Blue triangles show source term of the progress variable at reference condition $T_{o x}=1550 \mathrm{~K}, T_{f}=300 \mathrm{~K}$, and $p=1.0$ bar. Red circles show CFPV results computed from chemical library at reference conditions and compressibility correction, Eq. (26).

turbations in temperature and pressure. Equation (26) is devised to model the effects of these perturbations. The rescaling of the source term given by Eq. (26) is illustrated in Fig. 7, which demonstrates that this rescaling performs quite well for relatively strong perturbations in temperature and pressure that are often present in high-speed flows due to shock waves and expansion fans. This simple a priori analysis indicates that the important effects associated with compressibility are captured relatively well by the proposed model. Further validations are presented in the next two sections.

It should be noted that combined perturbation of pressure and temperature does not change the conclusions of this study. The same conclusions are also obtained for different values of $\widetilde{Z^{\prime \prime 2}}$. 


\section{Application of the CFPV model for the case of a hydrogen jet in a supersonic cross-flow}

The combustion model is tested for the case of a jet in a supersonic crossflow (JICF) and compared with the experimental measurements of Gamba et al. [38] and Heltsley et al. [39]. The JICF configuration is a canonical flow often encountered in supersonic combustion. It is of relative simplicity, yet it retains many features of interest, such as three-dimensionality, separation and recirculation regions, wall effects, and vortical flows. Furthermore, it maintains a high level of practical relevance for engineering applications.

A wide body of work has been conducted to characterize and study this system, both at low and high speeds, especially under non-reacting conditions. Reacting transverse jets in the supersonic regime have, however, received much less attention, partially due to the requirements and complexity to sustain combustion in a supersonic flow [39-42].

Several numerical investigations have been conducted on the JICF configuration. Direct numerical simulations at very low Mach number have been used to study jet mixing, flow structures, and jet trajectories [43-46]. In recent years, several numerical simulations of under-expanded jets in supersonic cross-flow have been performed. However, a vast majority of these simulations have been devoted to inert flows [47-50]. For instance, Boles et al. [47] have shown that in their simulations, a hybrid LES/RANS method was more accurate than a simple RANS approach; however, even in the hybrid approach, jet penetration and mixing showed some discrepancy with exper-

imental measurements. Kawai and Lele [49] used LES to study jet mixing showing good agreement with experimental data. On the other hand, numer- 
ical studies of reacting jets in supersonic cross-flow are rare. Won et al. [51] used the detached eddy simulation (DES) approach with a finite-rate chemistry model. Although their numerical results show overall good agreement with experimental observations, there are some inconsistencies in quantitative comparisons, e.g. lower jet penetration and narrower bandwidth. Also, the turbulent structures in the jet shear layer were very diffusive, likely due to the dissipative nature of their numerical scheme or their turbulence model.

\subsection{Flow configuration}

The experimental measurements used in the present study [38] have been conducted in an expansion tube at the end of which a flat plate (corresponding to $y=0$ ) was mounted parallel to the free stream direction. The final aero-thermodynamic state of the test gas could be adjusted by changing the

filling pressure of each of the three sections of the expansion tube, allowing for a wide range of conditions to be explored. An under-expanded hydrogen jet was injected perpendicularly into the free stream from a contoured nozzle with diameter $d=2 \mathrm{~mm}$ in the flat plate. The nozzle was located at a distance of $66.7 \mathrm{~mm}$ downstream of the flat plate leading edge. Different diagnostics techniques, such as Schlieren, $\mathrm{OH}^{*}$ chemiluminescence, $\mathrm{OH}$ planar laser-induced fluorescence (PLIF) imaging, and surface pressure measurements were used to investigate mixing, ignition, combustion, flame holding, and the stabilization processes of a hydrogen flame in the supersonic regime.

A single free stream condition representative of the conditions in a scramjet engine of a hypersonic vehicle at Mach 8 flight and $30 \mathrm{~km}$ altitude is used in this computational study. The corresponding nominal conditions of the free-stream flow, estimated from direct observations on directly measurable 
quantities such as Mach number and static pressure, are characterized by a static pressure $P=40 \mathrm{kPa}$, Mach number $M=2.4$, and static temperature $T=1550 \mathrm{~K}$, corresponding to a speed of $U=1800 \mathrm{~m} / \mathrm{s}$. Indirect measurements indicated that the incoming boundary layer was laminar in the upstream region of the jet. Because of the very short test time of the experiment, i.e. $0.1-0.5 \mathrm{~ms}$, where (quasi)-steady test gas conditions were observed, the temperature of the plate is assumed to remain constant and an isothermal boundary condition with $T_{w}=300 \mathrm{~K}$ is applied.

Global characteristics of a JICF, such as penetration, have been found to primarily depend on a single parameter, the jet-to-crossflow momentum flux ratio $J$ defined as

$$
J=\frac{\rho u_{H_{2}}^{2}}{\rho u_{\text {air }}^{2}} .
$$

As experimental measurements have shown [38], the combustion characteristics are also strongly dependent on $J$. In this computational study, the highest $J$ value studied in the experiment [38], i.e. $J=5.0$, is chosen. For this $J$ value, the total pressure, total temperature, and Reynolds number in the plenum of the injection nozzle are $P_{0}=2024 \mathrm{kPa}, T_{0}=300 \mathrm{~K}$, and $\mathrm{Re}_{d}=507400$, respectively.

\subsection{Numerical implementation}

The proposed combustion model has been implemented in both a RANS and an LES solver. The parallel LES solver is based on an unstructured finite volume method with explicit third-order Runge-Kutta time integration. The spatial discretization relies on a hybrid central and ENO method, in which a shock sensor is used to identify the cells where the ENO scheme should be 
applied. The sub-grid terms in Eqs. (1)-(6) are modeled using the dynamic procedure [27]. A body-fitted block refined unstructured mesh [52] is used for all of the simulations in this study. Several embedded zones of refinement are defined, to provide sufficient resolution in regions where gradients are large, e.g., in boundary layers and fuel/air mixing layers. The simulations are performed using a coarse mesh with 21 million cells, and a fine mesh with 46 million cells to evaluate the grid independence of the solution. The results presented in this study correspond to the fine mesh.

The RANS solver used for the solution of the compressible Navier-Stokes equations is based on a finite volume formulation and implicit time-integration on arbitrary polyhedral meshes [53]. The Euler fluxes are computed with a HLLC $[54,55]$ approximate Riemann solver. Second-order accuracy is typically achieved by computing the states at each side of a given cell face using second-order interpolation and then applying the same flux evaluation scheme to the reconstructed states. Gradients are limited with a modified version of Barth and Jespersen slope limiter [56-58]. The system of Eqs. (1)-(6) is solved fully coupled and the resulting large sparse system (the Jacobian matrices are obtained using first-order discretization) is solved with the generalized minimal residual method (GMRES) using the freely available linear solver package PETSc [59]. Different turbulence models are implemented, but all results shown here are computed with the two-equation turbulence model $k-\omega$ SST [26]. The turbulent Schmidt number for the scalars is set to $\mathrm{Sc}_{t}=0.5$ and the turbulent Prandtl number is $\operatorname{Pr}_{t}=0.5$.

In both cases, a very accurate hydrogen chemistry mechanism based on an improved GRI3.0 chemical kinetics mechanism [60,61] has been used. 
This mechanism is based on nine species, i.e. $\mathrm{O}_{2}, \mathrm{H}_{2}, \mathrm{O}, \mathrm{H}, \mathrm{OH}, \mathrm{H}_{2} \mathrm{O}, \mathrm{HO}_{2}$, $\mathrm{H}_{2} \mathrm{O}_{2}$ and $\mathrm{N}_{2}$, and 28 reactions, and does not include nitrogen chemistry.

\subsection{Results}

In this section, instantaneous LES flow fields are qualitatively compared to experimental data obtained through PLIF imaging of the $\mathrm{OH}$ radical first, then average LES fields are compared to RANS results and average experimental measurements.

It should be mentioned that the experiments only report the $\mathrm{OH}$ fluorescence signals, and, although, the experimental OH PLIF signal is correlated with the hydroxyl radical concentration, it is a non-linear function of other parameters like mole fractions of all species and more importantly pressure and temperature of the mixture. Moreover, the $\mathrm{OH}$ concentration in the LES results is interpolated from a pre-computed flamelet library, which is constructed at a constant background pressure, oxidizer and fuel temperatures. As shown in Fig. 2, OH mass fractions vary up to a factor of two due to compressibility effects. In this respect, the following simulation results should be interpreted only in a qualitative sense.

\subsubsection{Instantaneous flow-fields}

Figure 8 shows a side view of the instantaneous $\mathrm{OH}$ distribution for the symmetry plane in a comparison of LES results (top) and experimental measurements (bottom). Two distinct regions of strong combustion are observed, i.e. at the upper shear layer between the air and fuel streams and in the boundary layer. Notice that burning starts in the recirculation region upstream of the jet exit. Length and height of this recirculating zone, which 

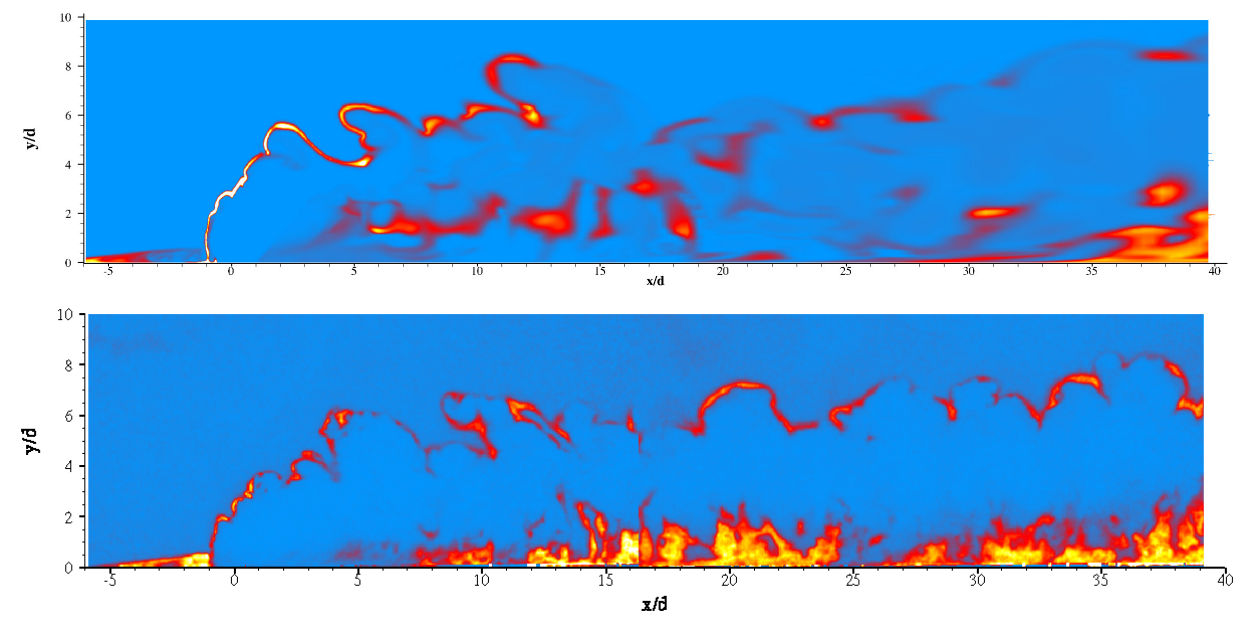

Figure 8: Comparison of instantaneous $\mathrm{OH}$ mass fraction from LES (top) with experimental OH PLIF signal (bottom) in the symmetry plane $z=0$. Contours for LES results are from $\widetilde{Y}_{\mathrm{OH}}=0$ (blue) to $\widetilde{Y}_{\mathrm{OH}}=0.024$ (white).

are about $6 d$ and $0.5 d$, respectively, are in good agreement with the experimental data [38]. In the experiment, burning is seen in the boundary layer at a distance of about $5 d$ downstream of the jet, and the LES results also show higher concentration of $\mathrm{OH}$ in the lower shear layer around the same location.

A very strong fuel cooling takes place just downstream of the jet as the fuel expands out of the nozzle, explaining the weak burning in the boundary layer before $x \sim 5 d$. To demonstrate the importance of the temperature correction (Eq. (26)), a simulation has been performed without temperature rescaling of the progress variable source term. Figure 9 displays higher concentration of $\mathrm{OH}$ in the lower shear layer, which is inconsistent with the experiment and LES results of Fig. 8.

Computed instantaneous $\mathrm{OH}$ mass fraction distributions in different planes 


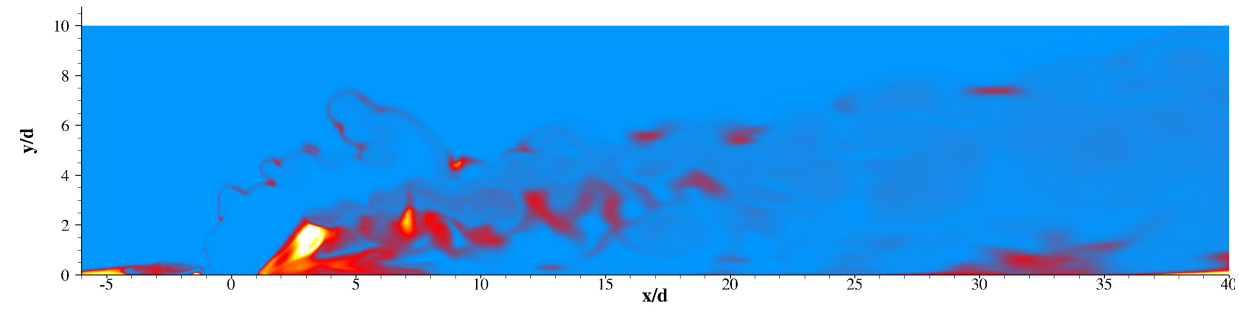

Figure 9: Instantaneous $\mathrm{OH}$ mass fraction without applying temperature correction for the source term of the progress variable (Eq. (26)). Contours are from $\tilde{Y}_{\mathrm{OH}}=0$ (blue) to $\tilde{Y}_{\mathrm{OH}}=0.024$ (white).

parallel to the plate are shown in Fig. 10. Burning is observed in a large part of the boundary layer in Figs. 10a and 10b, in good agreement with the experimental data for $y=0.5 d$ shown in Fig. 11 [38]. While Fig. 10c still shows some traces of burning in the boundary layer at $y=d$, burning is only present in the mixing layer at $y=3 d$ (Fig. 10d). Figure 11 shows the experimental OH PLIF signal in the $y=0.5 d$ plane, which corresponds to the LES results shown in Fig. 10b. The borders of the burning region in the boundary layer and the recirculation region are in good agreement with the experimental data.

Discrepancies between LES and experiment are also observed. For instance, the comparison in Fig. 8 shows that the burning in the boundary layer downstream of the jet exit seems to be closer to the wall in the experiment. This is also observed in the time-averaged data in section 5.3.2. The two aspects discussed earlier in this section, the difference between experimental $\mathrm{OH}$ PLIF signal and $\mathrm{OH}$ mass fraction and the fact that $\mathrm{OH}$ is more sensitive to the compressibility effects as shown in Fig. 2, could partly ex- 




(a) $y=0.25 d$

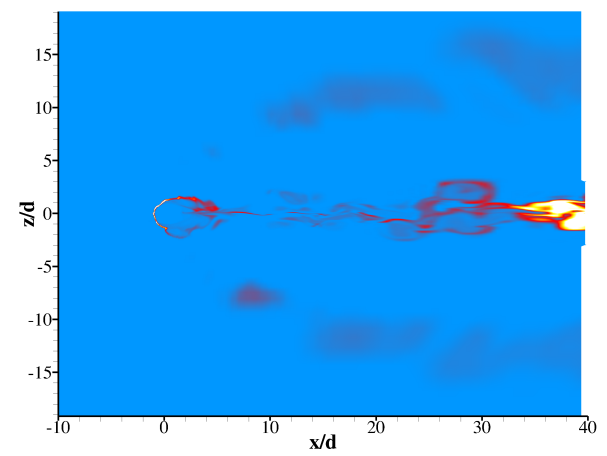

(c) $y=d$

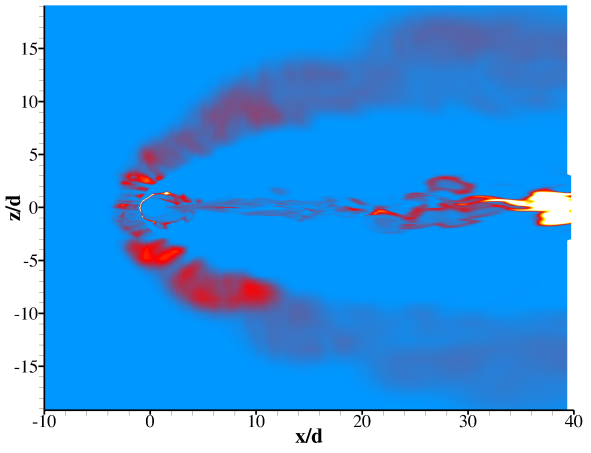

(b) $y=0.5 d$

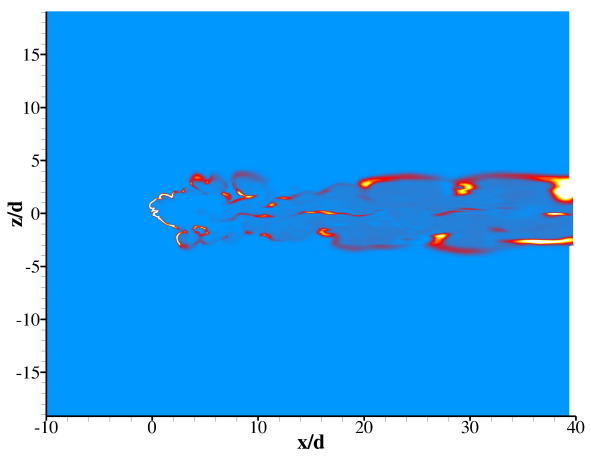

(d) $y=3 d$

Figure 10: Instantaneous $\mathrm{OH}$ mass fraction computed from LES in different planes parallel to the plate. Contours are from $\widetilde{Y}_{\mathrm{OH}}=0$ (blue) to $\widetilde{Y}_{\mathrm{OH}}=0.024$ (white). 


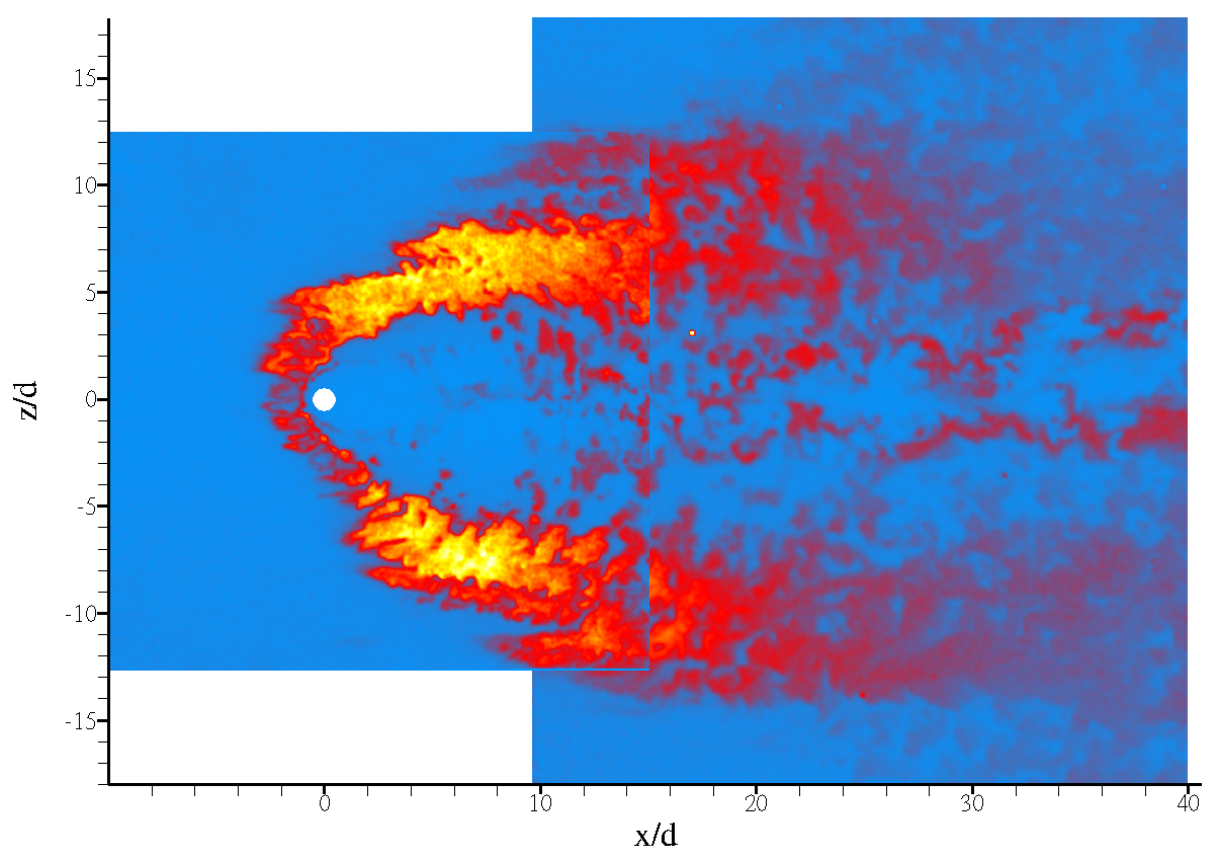

Figure 11: Experimental OH PLIF signal in the plane $y=0.5 d$. Contours are from blue to white.

plain these discrepancies. However, other modeling assumptions might also contribute to the discrepancies, e.g., closure assumptions in Eq. (5), and the presumed PDF assumptions. Auto-ignition of the fuel jet in this region could be another explanation, as it cannot be predicted accurately by the proposed method, and needs additional modifications [20]. A more detailed analysis is, however, required to investigate the combustion regime in this region.

\subsubsection{Time-averaged flow-fields}

In this section, statistics from LES, obtained by time-averaging instantaneous flow-fields are compared to steady RANS results and experimental 
data averaged over eleven single images. It should be noted that the number of samples that contributed to the experimental average is insufficient to yield converged statistics. Nonetheless, they provide a useful approximation of the general flow features.

The time-averaged LES and RANS results at the $y=0.5 d$ planes are juxtaposed in Fig. 12. The LES results show a wide burning zone in the boundary layer, which is in good agreement with the experimental observations shown in Fig. 13. However, this burning zone is much narrower in the RANS case. This is due to the smaller upstream recirculation zone predicted by the RANS simulations, which can also be seen in Fig. 14. The smaller recirculation zone reduces the amount of fuel transported around the jet in the boundary layer and, thereby, the extent of the reactive region. Note that the smaller recirculation zone predicted by RANS is partly due to the laminar character of the boundary layer upstream of the jet, which is not correctly represented by the RANS simulations. Experimental measurements with a trip wire to enforce a turbulent incoming boundary layer have shown a much smaller recirculation zone [62]. It should be noted that, LES and RANS results predict chemical conversion close to the centerline, $z=0$, while the experimental measurements do not support this observation.

Figure 14 also illustrates the discrepancies between LES and RANS results. Not only the recirculation zone upstream of the jet is smaller in the RANS computation, but also the mixing layer between fuel and air is less diffusive. In the LES result, the mixing layer on the leeward side starts to burn around $x \sim 5 d$, which is consistent with the experiment. Burning in the experiment is, however, closer to the wall, as discussed above. 


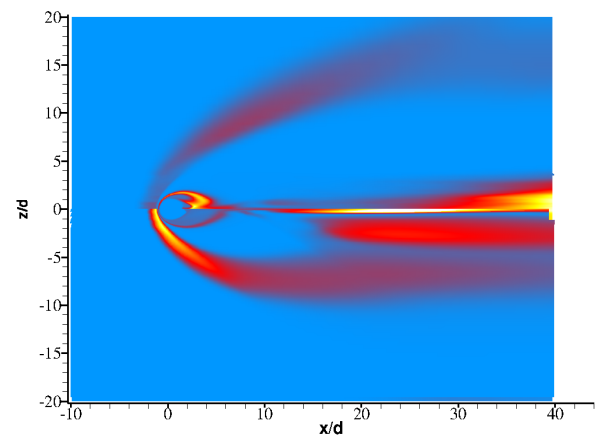

Figure 12: Side to side comparison between LES (top) and RANS (bottom) of time-averaged $\mathrm{OH}$ mass fraction and mixture fraction in the plane $y=0.5 d$. Contours are from $\widetilde{Y}_{\mathrm{OH}}=0$ (blue) to $\widetilde{Y}_{\mathrm{OH}}=0.022$ (white).

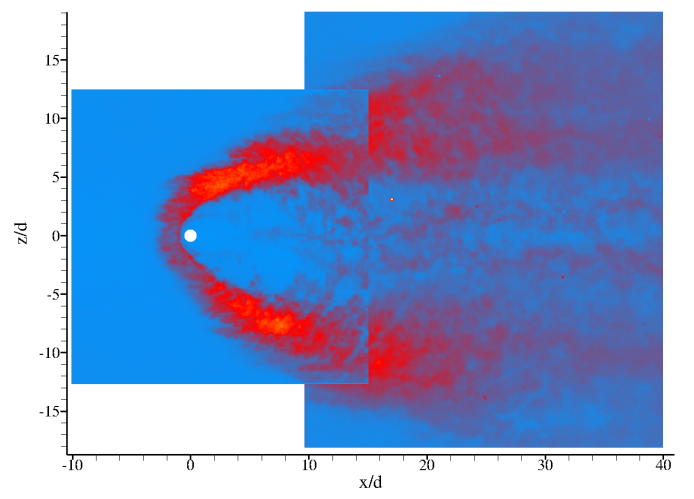

Figure 13: Eleven-frame average of the experimental OH PLIF signal in the $y=0.5 d$ plane. 

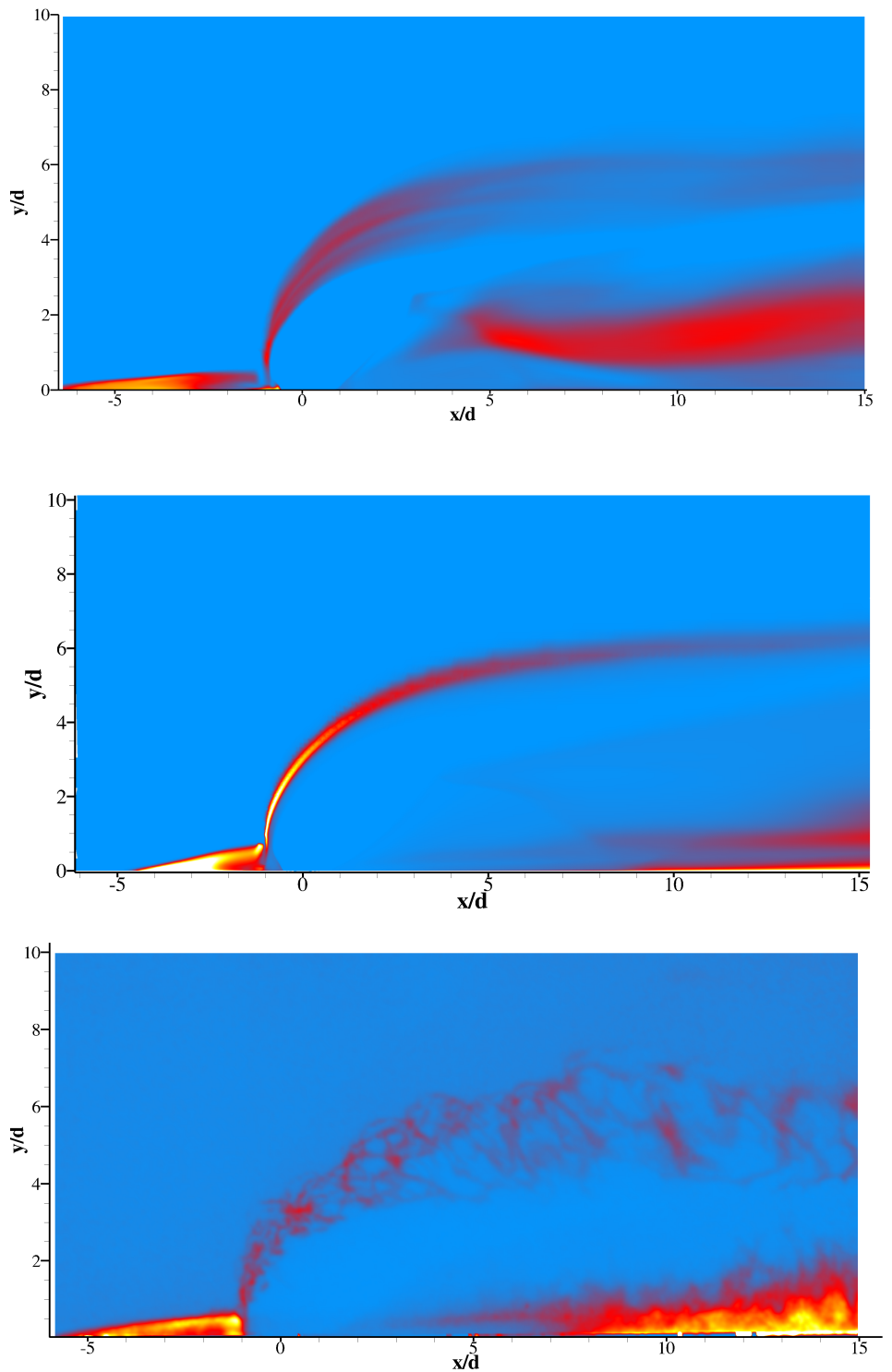

Figure 14: Time averaged $\mathrm{OH}$ mass fraction in the symmetry plane $z=0$; LES (top), RANS (middle), and eleven-frame average experimental OH PLIF measurement (bottom). Contours are from $\widetilde{Y}_{\mathrm{OH}}=0$ (blue) to $\widetilde{Y}_{\mathrm{OH}}=0.024$ (white) in LES and RANS. 



Figure 15: Time averaged mixture fraction in the symmetry plane $z=0$; LES (top) and RANS (bottom). Contours are from $\widetilde{Z}=0$ (blue) to $\widetilde{Z}=1$ (white).

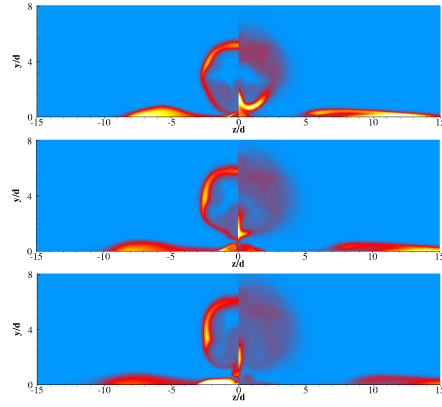

(a) $\mathrm{OH}$ mass fraction

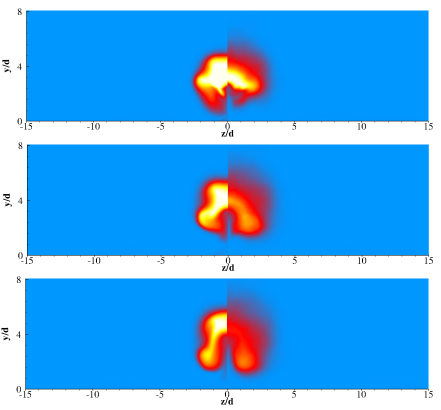

(b) mixture fraction



(c) water mass fraction

Figure 16: Side to side comparison between time averaged LES (right half) and RANS (left half) in the planes $x=4 d$ (top), $x=8 d$ (middle), and $x=12 d$ (bottom). Contours are from $\widetilde{Y}_{\mathrm{OH}}=0$ (blue) to $\widetilde{Y}_{\mathrm{OH}}=0.024$ (white), $\widetilde{Z}=0$ (blue), to $\widetilde{Z}=1$ (white), and $\widetilde{C}=0$ (blue), to $\widetilde{C}=0.25$ (white), respectively. 
Another difference between RANS and LES is illustrated in Fig. 15, where the time-averaged mixture fraction is compared. In particular, a much larger jet plume is predicted by the RANS simulation, indicating deficiencies of the RANS mixing model as already observed in Fig. 14. Stronger mixing could be achieved by reducing the value of the turbulent Schmidt number, but such an approach would require a problem-dependent calibration. To circumvent this issue, a more elaborate mixing model would be required.

Time averaged contours of the $\mathrm{OH}$ mass fraction, mixture fraction, and water mass fraction (progress variable) at three different planes normal to the cross-flow at the locations $x=4 d, x=8 d$, and $x=12 d$, are shown in Fig. 16. Again, burning is observed in the boundary layer and in the shear layer between the fuel and air streams, while the core of the hydrogen jet does not burn. This figure further illustrates the lower mixing rate demonstrated by the RANS simulations.

\section{Sensitivity analysis}

The CFPV model represents a low dimensional manifold parameterized

with $\widetilde{Z}, \widetilde{Z^{\prime \prime 2}}$, and $\widetilde{C}$, and internal energy and pressure with a flamelet library evaluated at a reference internal energy and pressure and an expansion around this base solution in terms of these parameters. At what condition the base solution is computed is to some degree arbitrary, and, therefore, the sensitivity to this choice should be determined. If the sensitivity of the solution to this choice was large, the model accuracy would depend on it. In other words, a perfect model would be totally insensitive to the reference parameters. Also, if the model was sensitive to these values, more accurate 
guidelines for this choice would have to be provided.

The only user-specified parameters in the CFPV model are the oxidizer temperature $T_{o x}^{r}$, fuel temperature $T_{f}^{r}$, and background pressure $p_{t b}^{r}$. The flamelet equations are then solved subject to these parameters, and necessary variables (e.g. $T_{0}, a_{\gamma}, a_{\rho}$, and $T_{a}$ ) are computed and tabulated. Consequently, all variables are functions of these input parameters, which are statistically independent. It should be emphasized that pressure and temperature are actually extremely correlated in compressible flows. However, $T_{o x}^{r}, T_{f}^{r}$, and $p_{t b}^{r}$ are not correlated; these quantities are reference parameters, and are thus statistically independent.

The proposed CFPV model attempts to partly account for the compressibility effects and viscous heating through the use of compressibility corrections (see section 3.3). A comparison of Figs. 1 and 2 reveals that the flamelet solutions are much more sensitive to the oxidizer temperature than the fuel temperature; therefore, only variations in the oxidizer temperature and background pressure are considered here.

Since the results of an LES or RANS simulation using the CFPV model depend on the choice of $p_{t b}^{r}$ and $T_{o x}^{r}$, these parameters shall now be treated as independent random variables influencing the solution. To quantitatively assess the impact of the choice of these parameters, the statistics of the solution with respect to variations in the parameters can be computed and the resulting expectation is compared with the standard deviation. The solution can be called insensitive to the choice of the parameters, if the standard deviation is small compared to the expectation.

The statistical properties of any variable (at a given $\widetilde{Z}, \widetilde{Z^{\prime \prime 2}}$, and $\widetilde{C}$ ), can 
be computed, if the joint probability density function (PDF) of the parameters (i.e, $\left.p_{t b}^{r}, T_{o x}^{r}\right)$ is known; sensitivity of any variable $f\left(T_{o x}^{r}, p_{t b}^{r}\right)$ to the variations of the input parameters can then be quantified by computing its expectation and variance,

$$
\begin{gathered}
\mu_{f}=\iint f\left(T_{o x}^{r}, p_{t b}^{r}\right) P\left(T_{o x}^{r}, p_{t b}^{r}\right) d T_{o x}^{r} d p_{t b}^{r}, \\
\sigma_{f}^{2}=\iint\left[f\left(T_{o x}^{r}, p_{t b}^{r}\right)-\mu_{f}\right]^{2} P\left(T_{o x}^{r}, p_{t b}^{r}\right) d T_{o x}^{r} d p_{t b}^{r},
\end{gathered}
$$

where $f$ could be any variable, $\mu_{f}$ is its expectation, and $\sigma_{f}^{2}$ its variance. $P$ denotes the joint PDF of the oxidizer temperature and background pressure, such that,

$$
P\left(T_{o x}^{r}, p_{t b}^{r}\right)=P\left(T_{o x}^{r}\right) P\left(p_{t b}^{r}\right)
$$

because all the input parameters of the CFPV method are independent. Note that $f$ is a function of space and time; this is considered here, but the dependency has not been explicitly denoted for simplicity. Moreover, the same notation has been used for the sample space variable and stochastic variable.

Quadrature rules can be used to numerically compute the integrals in Eqs. (28) and (29). The joint PDF in Eq. (30) is evaluated assuming a Gaussian distribution for the oxidizer temperature and background pressure. Equation (28) is then approximated using Gauss-Hermite quadrature,

$$
\mu_{f} \simeq \frac{1}{\pi} \sum_{i=1}^{N_{1}} \sum_{j=1}^{N_{2}} \omega_{i} \omega_{j} f\left(T_{o x i}^{r}, p_{t b j}^{r}\right)
$$


where $\omega_{i}$ are the Gauss-Hermite quadrature weights, and $N_{1}$ and $N_{2}$ are the number of sample points in the oxidizer temperature and background pressure, respectively. Equation (31) requires a total of $N_{1} N_{2}$ simulations of the problem, each with a different flamelet library. Variances can then be computed using the same quadrature rule,

$$
\sigma_{f}^{2} \simeq \frac{1}{\pi} \sum_{i=1}^{N_{1}} \sum_{j=1}^{N_{2}} \omega_{i} \omega_{j}\left[f\left(T_{o x i}^{r}, p_{t b j}^{r}\right)-\mu_{f}\right]^{2} .
$$

The mean and variance of the oxidizer temperature and pressure are estimated using the LES results of section 5.3. A total of nine simulations, three sample points in each direction, are performed with different flamelet libraries. Each circle in Fig. 17 represents one of these simulations where the horizontal and vertical axes show the background pressure and oxidizer temperature of the flamelet library, respectively. It should be mentioned that increasing the number of sample points does not significantly decrease the truncation error in Eqs. (31) and (32), because the Gauss-Hermite quadrature weights drop exponentially.

The expectation and standard deviation of the progress variable, using Eqs. (31) and (32), are plotted in Fig. 18 for RANS simulations. The standard deviation is at least one order of magnitude smaller than the expectation, which shows that the CFPV method is not very sensitive to variations in the background pressure and oxidizer temperature when the compressibility corrections introduced in section 3.3 are active.

Among these compressibility corrections, the rescaling of the source term of the progress variable, Eq. (26), plays a crucial role in increasing the fidelity of the CFPV method. To show the importance of this rescaling, we 


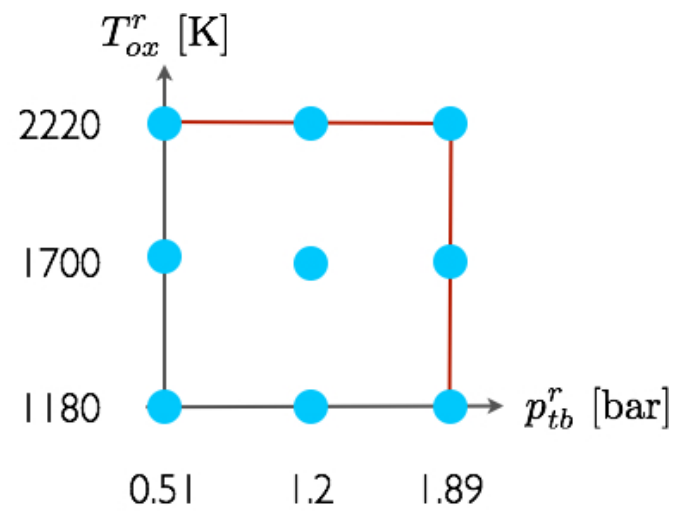

Figure 17: Each blue circle represents a sample point of the reference condition used to create the chemistry table based on the Gauss-Hermite quadrature points.

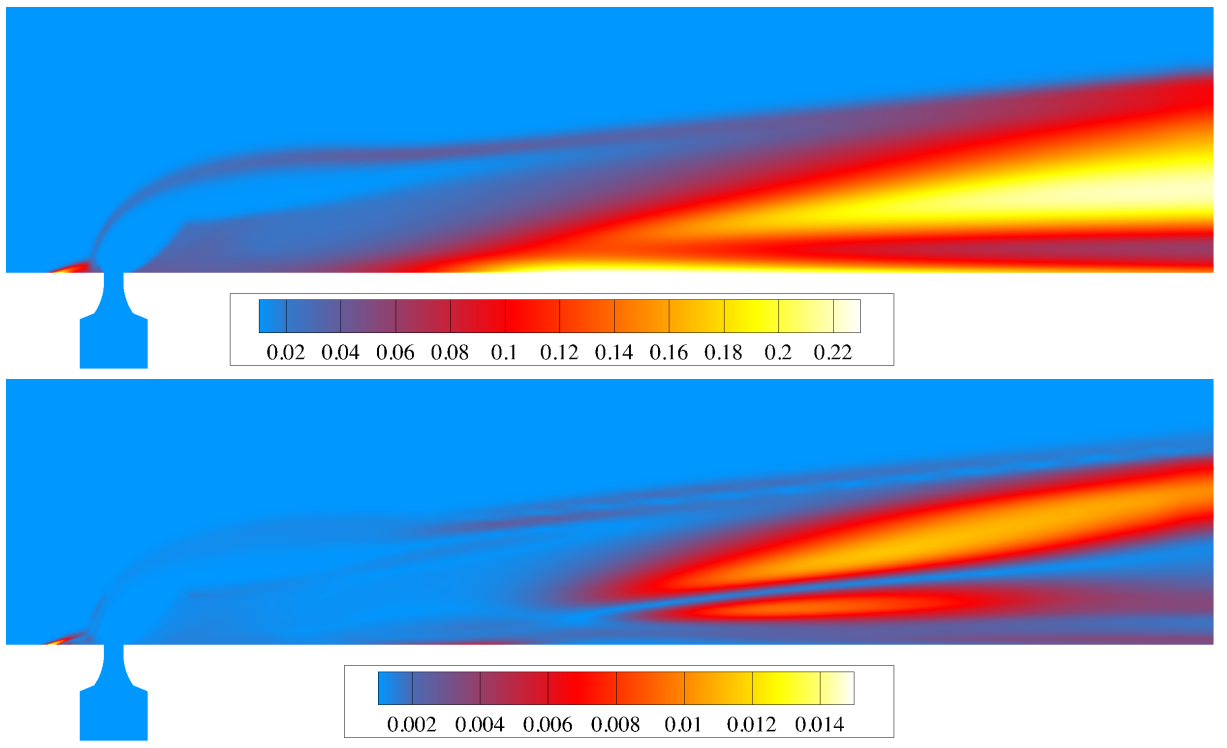

Figure 18: Expectation (top) and standard deviation (bottom) of the progress variable computed using RANS and Gauss-Hermite quadrature. 
performed RANS simulations for all nine sample points in Fig. 17, where the rescaling with Eq. (26) was deactivated. Figure 19 shows the expectation and standard deviation of the progress variable without using Eq. (26). The standard deviation in this case is almost of the same order of magnitude as the expectation. The comparison between Figs. 18 and 19 demonstrates that the CFPV model can account for the major part of the compressibility effects. To better observe the effects of Eq. (26), contours of the progress variable are plotted in Fig. 20 for the case with $T_{o x}^{r}=1180 \mathrm{~K}$ and $p_{t b}^{r}=1.2$ bar. When Eq. (26) is deactivated, the simulation does not show any burning region. On the other hand, using the compressibility correction for the source term of the progress variable leads to a flow-field which is consistent with experimental results. If the correction is not used, large errors are observed in the regions where compressibility is important, for example, where shock waves or expansion fans interact with the flame or in the boundary layer where viscous heating and heat losses are important.

\section{Conclusion}

An efficient flamelet-based combustion model for compressible flows has been introduced in this work. In this approach, only two or three additional scalar transport equations need to be solved, independently of the complexity of the reaction mechanism used in the simulations.

The CEFT model is the extension of the low Mach number flamelet/progress variable approach. The flamelet library in this approach includes all possible flamelet solutions, which can be represented by an S-shaped hypersurface. The memory requirement of a five-dimensional flamelet library is, however, 

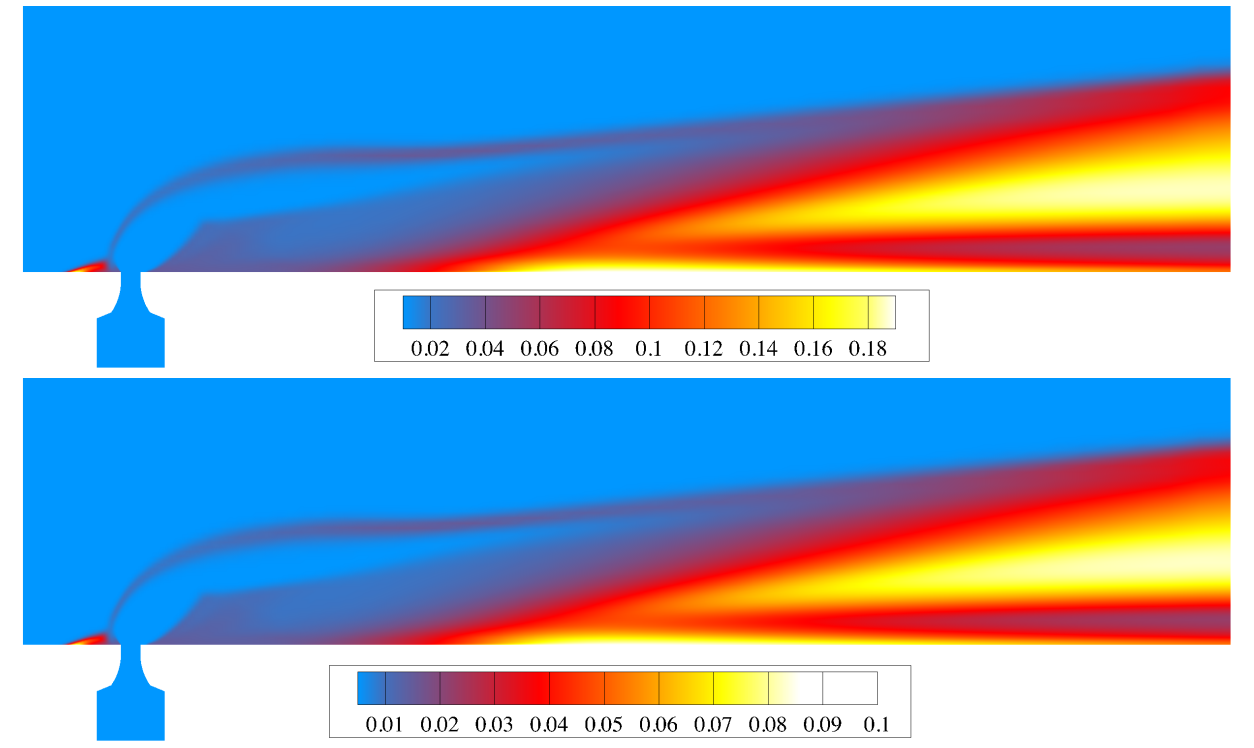

Figure 19: Same as Fig. 18, but without the compressibility correction of the source term of the progress variable (Eq. (26)).

prohibitive. The CFPV approach circumvents this by utilizing compressibility corrections around a flamelet library at the reference condition, thus keeping the dimension of the flamelet library the same as for the FPV model. Compressibility corrections have been introduced considering the physical behavior of each parameter. These compressibility corrections are validated in an a priori analysis. This analysis also reveals that mixture properties which are not function of temperature (e.g. the gas constant) have very small departure from the reference solution, whereas mixture properties that depend on temperature (e.g. specific heat ratio and source term of the progress variable) show higher sensitivity to the perturbations.

The model is then tested in both LES and RANS computations of an under-expanded hydrogen jet in a supersonic cross-flow. Comparisons with 


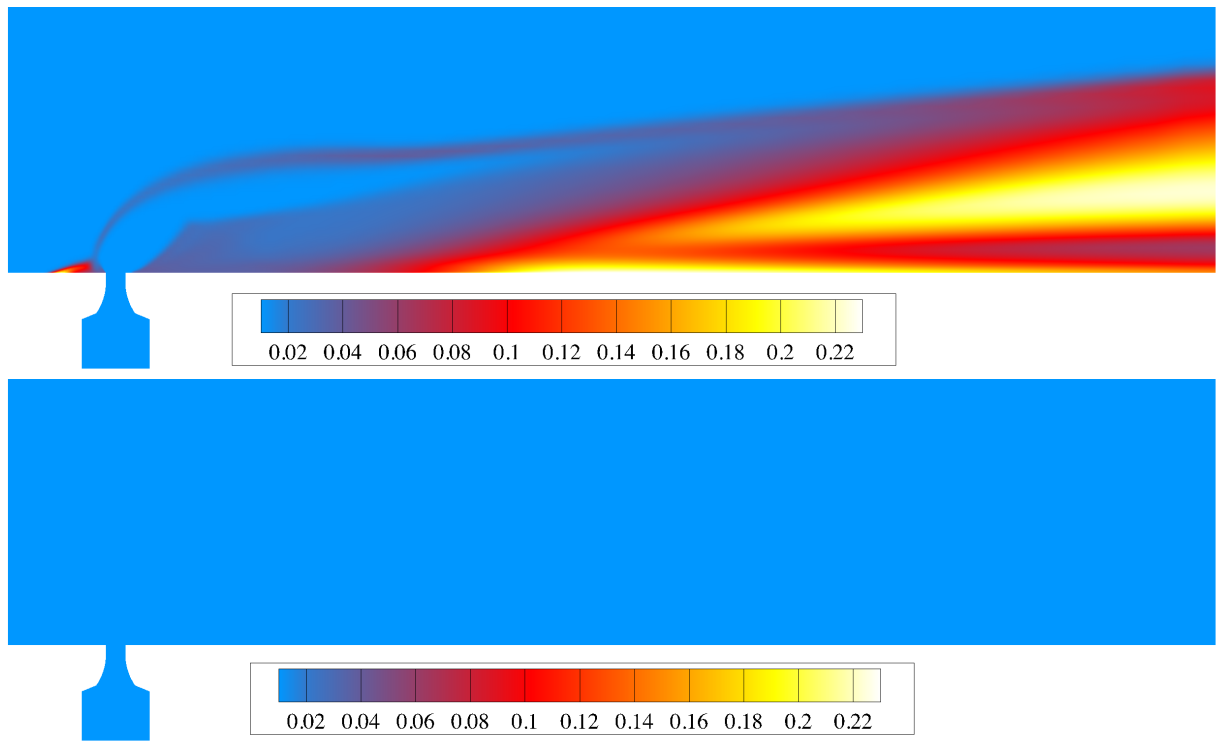

Figure 20: Contours of the progress variable in the symmetry plane $z=0$ for the case $T_{o x}^{r}=1180 \mathrm{~K}$ and $p_{t b}^{r}=1.2$ bar computed with RANS; the compressibility correction of the source term of progress variable (Eq. (26)) is activated (top) and deactivated (bottom). 
experimental measurements show relatively good agreement particularly for the LES results. Burning is observed upstream of the jet in the recirculation region, and in a big part of the boundary layer downstream of the jet exit, consistent with the experimental measurements. The burning recirculation region functions as a physical flame-holder, and presumably renders a more stable flame; this could result into a smaller scramjet by promoting ignition. Time-averaged LES results also show that discrepancies in the RANS results are likely due to deficiencies of the RANS mixing model. The state of the incoming boundary layer also plays an important role in the size of the recirculation zone.

A sensitivity study of the CFPV approach confirms the importance of the compressibility corrections, especially for the source term of the progress variable. This study shows that deactivating the compressibility correction of the source term of the progress variable, Eq. (26), produces a standard deviation, which is of the same order of magnitude as the expectation, whereas activating this correction results in a standard deviation, which is at least one order of magnitude smaller than the expectation.

It is important to note, however, that the proposed models suffer the same limitations of the low Mach number FPV approach. For instance, additional modifications should be considered in order to differentiate between regions where auto-ignition or unsteady effects are important. Also, the presumed PDF assumption of the mixture fraction and reaction progress parameter and the closure assumptions in the transport equation of the sub-grid mixture fraction variance have to be tested more rigorously. 


\section{Acknowledgments}

This material is based upon work supported by the Department of Energy under the Predictive Science Academic Alliance Program (PSAAP) at Stanford University, Award Number(s)DE-FC52-08NA28614. The authors acknowledge the following award for providing computing resources that have contributed to the research results reported within this paper:

MRI-R2: Acquisition of a Hybrid CPU/GPU and Visualization Cluster for Multidisciplinary Studies in Transport Physics with Uncertainty Quantification

http://www.nsf.gov/awardsearch/showAward.do?AwardNumber=0960306

This award is funded under the American Recovery and Reinvestment Act of 2009 (Public Law 111-5).

\section{References}

[1] J. M. Schramm, S. Karl, K. Hannemann, J. Steelant, Ground testing of the HyShot II scramjet configuration in HEG, AIAA Paper 20082547.

[2] M. K. Smart, N. E. Hass, A. Paull, Flight Data Analysis of the HyShot 2 Scramjet Flight Experiment., AIAA J. (2006) 2366-2375.

[3] J. M. Hank, J. S. Murphy, R. C. Mutzman, The X-51A scramjet engine flight demonstration program, AIAA Paper 2540 (2008) 2008.

[4] M. Bolender, J. Staines, D. Dolvin, HIFiRE 6: An Adaptive Flight Control Experiment, AIAA paper 252. 
[5] Y. Moule, V. Sabelnikov, A. Mura, Highly resolved numerical simulation of combustion in supersonic hydrogen-air coflowing jets, Combust. Flame, In Press 2014.

[6] D. Davidenko, I. Gokalp, E. Dufour, P. Magre, Numerical simulation of hydrogen supersonic combustion and validation of computational approach, AIAA Paper 2003-7033.

[7] K. Kumaran, V. Babu, Investigation of the effect of chemistry models on the numerical predictions of the supersonic combustion of hydrogen, Combust. Flame 156 (4) (2009) 826-841.

[8] D. Chakraborty, P. Paul, H. Mukunda, Evaluation of combustion models for high speed $\mathrm{H}_{2}$ /air confined mixing layer using DNS data, Combust. Flame 121 (1) (2000) 195-209.

[9] R. Baurle, S. Girimaji, Assumed PDF turbulence-chemistry closure with temperature-composition correlations, Combust. Flame 134 (1) (2003) $131-148$.

[10] S. Karl, K. Hannemann, A. Mack, J. Steelant, CFD analysis of the HyShot II scramjet experiments in the HEG shock tunnel, AIAA Paper 2008-2548.

[11] R. Baurle, A. Hsu, H. Hassan, Assumed and evolution probability density functions in supersonic turbulent combustion calculations, $J$. Propul. Power 11 (6) (1995) 1132-1138. 
[12] H. Möbus, P. Gerlinger, D. Brüggemann, Scalar and joint scalarvelocity-frequency Monte Carlo PDF simulation of supersonic combustion, Combust. Flame 132 (1) (2003) 3-24.

[13] H. Koo, P. Donde, V. Raman, A quadrature-based LES/transported probability density function approach for modeling supersonic combustion, Proc. Combust. Inst. 33 (2) (2011) 2203-2210.

[14] P. Donde, H. Koo, V. Raman, A multivariate quadrature based moment method for LES based modeling of supersonic combustion, J. Comput. Phys. 231 (17) (2012) 5805-5821.

[15] F. Genin, B. Chernyavsky, S. Menon, Large eddy simulation of scramjet combustion using a subgrid mixing/combustion model, AIAA Paper 2003-7035.

[16] C. D. Ghodke, J. J. Choi, S. Srinivasan, S. Menon, Large eddy simulation of supersonic combustion in a cavity-strut flameholder, AIAA Paper 2011-323.

[17] N. Peters, Turbulent combustion, Cambridge University Press, 2000.

[18] H. Pitsch, Large-eddy simulation of turbulent combustion, Annu. Rev. Fluid Mech. 38 (2006) 453-482.

[19] C. D. Pierce, P. Moin, Progress-variable approach for large-eddy simulation of non-premixed turbulent combustion, J. Fluid Mech. 504 (2004) 73-97. 
[20] M. Ihme, C. M. Cha, H. Pitsch, Prediction of local extinction and re-ignition effects in non-premixed turbulent combustion using a flamelet/progress variable approach, Proc. Combust. Inst. 30 (1) (2005) 793-800.

[21] M. Berglund, C. Fureby, LES of supersonic combustion in a scramjet engine model, Proc. Combust. Inst. 31 (2) (2007) 2497-2504.

[22] M. Oevermann, Numerical investigation of turbulent hydrogen combustion in a SCRAMJET using flamelet modeling, Aerosp. Sci. Technol. 4 (7) (2000) 463-480.

[23] S. Kumar, T. Tamaru, Computation of turbulent reacting flow in a jet assisted ram combustor, Comput. Fluids 26 (2) (1997) 117-133.

[24] R. Vicquelin, B. Fiorina, S. Payet, N. Darabiha, O. Gicquel, Coupling tabulated chemistry with compressible CFD solvers, Proc. Combust. Inst. 33 (1) (2011) 1481-1488.

[25] R. B. Bird, W. E. Stewart, E. N. Lightfoot, Transport phenomena, Wiley. com, 2007.

[26] F. R. Menter, Two-equation eddy-viscosity turbulence models for engineering applications, AIAA J. 32 (8) (1994) 1598-1605.

[27] P. Moin, K. Squires, W. Cabot, S. Lee, A dynamic subgrid-scale model for compressible turbulence and scalar transport, Phys. Fluids 3 (1991) 2746 . 
[28] A. Saghafian, High-fidelity simulations and modeling of compressible reacting flows, Ph.D. thesis, Stanford University, 2014.

[29] M. Ihme, Pollutant formation and noise emission in turbulent nonpremixed flames, Ph.D. thesis, Stanford University, 2007.

[30] C. D. Pierce, Progress-variable approach for large-eddy simulation of turbulent combustion, Ph.D. thesis, Stanford University, 2001.

[31] N. Peters, Laminar diffusion flamelet models in non-premixed turbulent combustion, Progress in energy and combustion science 10 (3) (1984) 319-339.

[32] M. Ihme, H. Pitsch, Prediction of extinction and re-ignition in nonpremixed turbulent flames using a flamelet/progress variable model: 1 . A priori study and presumed PDF closure, Combust. Flame 155 (1) (2008) 70-89.

[33] H. Pitsch, Modellierung der Zündung und Schadstoffbildung bei der diesel- motorischen Verbrennung mit Hilfe eines interaktiven FlameletModells, Ph.D. thesis, RWTH Aachen, 1998.

[34] L. Wang, H. Pitsch, K. Yamamoto, A. Orii, An efficient approach of unsteady flamelet modeling of a cross-flow-jet combustion system using LES, Combust. Theory Model. 15 (6) (2011) 849-862.

[35] V. Mittal, H. Pitsch, A flamelet model for premixed combustion under variable pressure conditions, Proc. Combust. Inst. 34 (2) (2013) 29953003. 
[36] G. Ribert, O. Gicquel, N. Darabiha, D. Veynante, Tabulation of complex chemistry based on self-similar behavior of laminar premixed flames, Combust. Flame 146 (4) (2006) 649-664.

[37] K. Wang, G. Ribert, P. Domingo, L. Vervisch, Self-similar behavior and chemistry tabulation of burnt-gas diluted premixed flamelets including heat-loss, Combust. Theory Model. 14 (4) (2010) 541-570.

[38] M. Gamba, M. G. Mungal, R. K. Hanson, Ignition and near-wall burning in transverse hydrogen jets in supersonic crossflow, AIAA Paper (2011) $4-7$.

[39] W. N. Heltsley, J. A. Snyder, C. C. Cheung, M. Mungal, R. K. Hanson, Combustion stability regimes of hydrogen jets in supersonic crossflow, AIAA Paper 5401.

[40] M. Lee, B. McMillin, J. Palmer, R. Hanson, Planar fluorescence imaging of a transverse jet in a supersonic crossflow, J. Propul. Power 8 (4) (1992) 729-735.

[41] B. McMillin, J. Seitzman, R. Hanson, Comparison of NO and OH planar fluorescence temperature measurements in scramjet model flowfield, AIAA J. 32 (10) (1994) 1945-1952.

[42] A. Ben-Yakar, R. K. Hanson, Supersonic combustion of cross-flow jets and the influence of cavity flame-holders, AIAA Paper 484 (1999) 1999.

[43] S. Muppidi, K. Mahesh, Study of trajectories of jets in crossflow using direct numerical simulations, J. Fluid Mech. 530 (5) (2005) 81-100. 
[44] S. Muppidi, K. Mahesh, Direct numerical simulation of round turbulent jets in crossflow, J. Fluid Mech. 574 (2007) 59-84.

[45] R. Sau, K. Mahesh, Optimization of pulsed jets in crossflow, J. Fluid Mech. 653 (2010) 365.

[46] R. Grout, A. Gruber, C. Yoo, J. Chen, Direct numerical simulation of flame stabilization downstream of a transverse fuel jet in cross-flow, Proc. Combust. Inst. 33 (1) (2011) 1629-1637.

[47] J. A. Boles, J. R. Edwards, R. A. Bauerle, Large-eddy/Reynoldsaveraged Navier-Stokes simulations of sonic injection into Mach 2 crossflow, AIAA J. 48 (7) (2010) 1444-1456.

[48] F. Génin, S. Menon, Dynamics of sonic jet injection into supersonic crossflow, J. Turb. 182 (11).

[49] S. Kawai, S. K. Lele, Large-eddy simulation of jet mixing in supersonic crossflows, AIAA J. 48 (9) (2010) 2063-2083.

[50] B. Fiorina, S. K. Lele, Numerical investigation of a transverse jet in a supersonic crossflow using large eddy simulation, AIAA Paper 20063712 .

[51] S.-H. Won, I.-S. Jeung, B. Parent, J.-Y. Choi, Numerical investigation of transverse hydrogen jet into supersonic crossflow using detached-eddy simulation, AIAA J. 48 (6) (2010) 1047-1058.

[52] G. A. Brès, J. W. Nichols, S. K. Lele, F. E. Ham, R. H. Schlinker, R. A. 
Reba, J. C. Simonich, Unstructured Large Eddy Simulation of a Hot Supersonic Over-Expanded Jet with Chevrons, AIAA Paper 2012-2213.

[53] R. Pecnik, V. E. Terrapon, F. Ham, G. Iaccarino, H. Pitsch, Reynoldsaveraged navier-stokes simulations of the hyshot II scramjet, AIAA J. 50 (8) (2012) 1717-1732.

[54] E. F. Toro, M. Spruce, W. Speares, Restoration of the contact surface in the HLL-Riemann solver, Shock waves 4 (1) (1994) 25-34.

[55] P. Batten, M. Leschziner, U. Goldberg, Average-state Jacobians and implicit methods for compressible viscous and turbulent flows, J. Comput. Phys. 137 (1) (1997) 38-78.

[56] T. J. Barth, D. C. Jespersen, The design and application of upwind schemes on unstructured meshes, AIAA Paper 484.

[57] V. Venkatakrishnan, Convergence to steady state solutions of the Euler equations on unstructured grids with limiters, J. Comput. Phys. 118 (1) (1995) 120-130.

[58] R. Pecnik, V. Terrapon, F. Ham, G. Iaccarino, Full system scramjet simulation, Annual Research Briefs of the Center for Turbulence Research

[59] B. Satish, K. Buschelman, V. Eijkhout, W. Gropp, D. Kaushik, M. Knepley, L. McInnes, B. Smith, H. Zhang, PETSc Web page URL http://www.mcs.anl.gov/petsc. 
[60] R. W. Bates, D. M. Golden, R. K. Hanson, C. T. Bowman, Experimental study and modeling of the reaction $\mathrm{H}+\mathrm{O}_{2}+\mathrm{M} \rightarrow \mathrm{HO}_{2}+\mathrm{M}\left(\mathrm{M}=\mathrm{Ar}, \mathrm{N}_{2}\right.$, $\left.\mathrm{H}_{2} \mathrm{O}\right)$ at elevated pressures and temperatures between 1050 and $1250 \mathrm{~K}$, Phys. Chem. 3 (12) (2001) 2337-2342.

[61] J. T. Herbon, R. K. Hanson, D. M. Golden, C. T. Bowman, A shock tube study of the enthalpy of formation of $\mathrm{OH}$, Proc. Combust. Inst. 29 (1) (2002) 1201-1208.

[62] M. Gamba, V. E. Terrapon, A. Saghafian, M. G. Mungal, H. Pitsch, Large-eddy/Reynolds-averaged Navier-Stokes simulations of sonic injection into Mach 2 crossflow, Annual research briefs, Center for Turbulence Research (2011) 259-272. 
Figure 1a

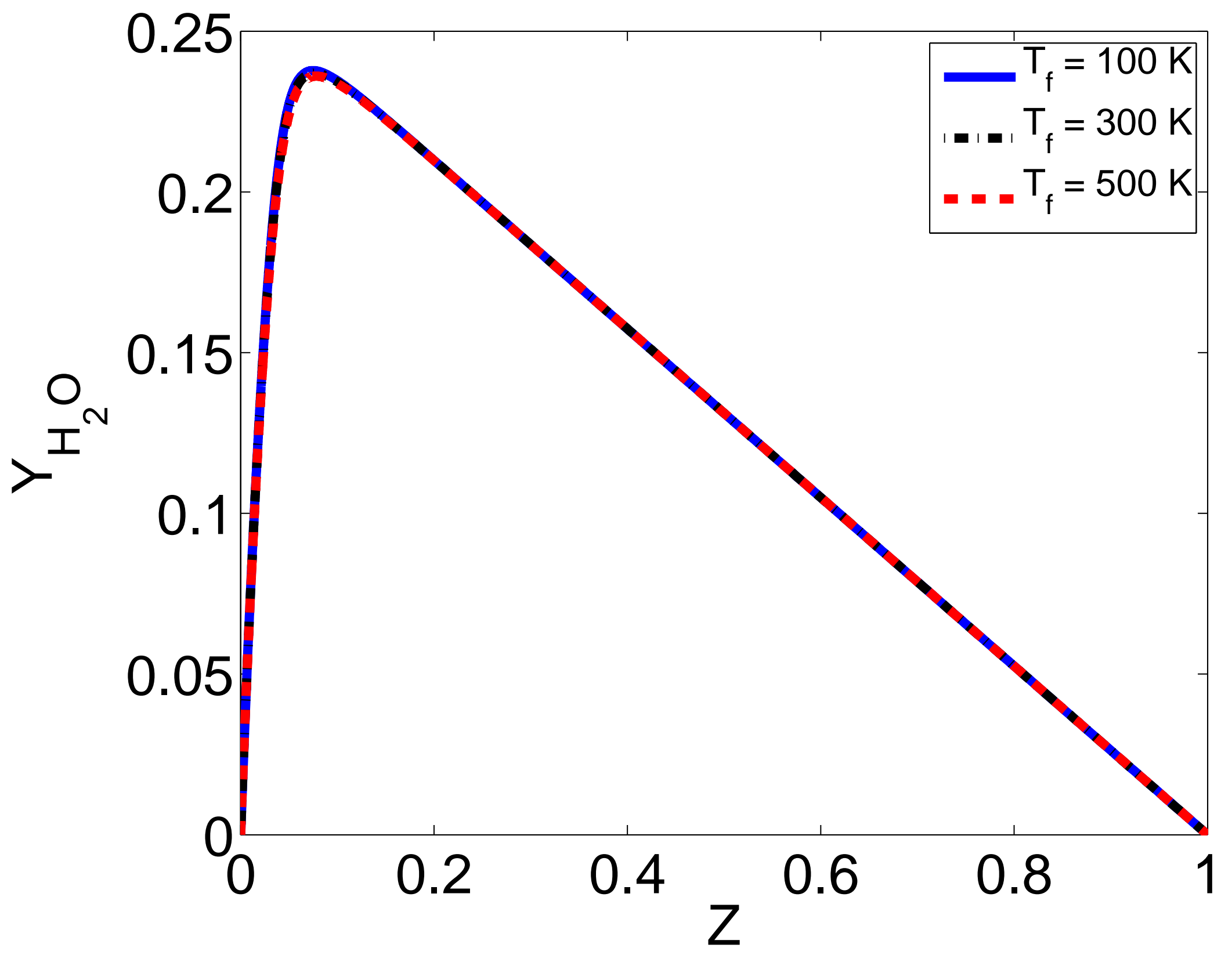




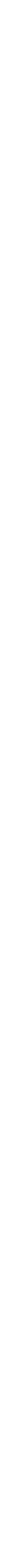

Figure $1 b$

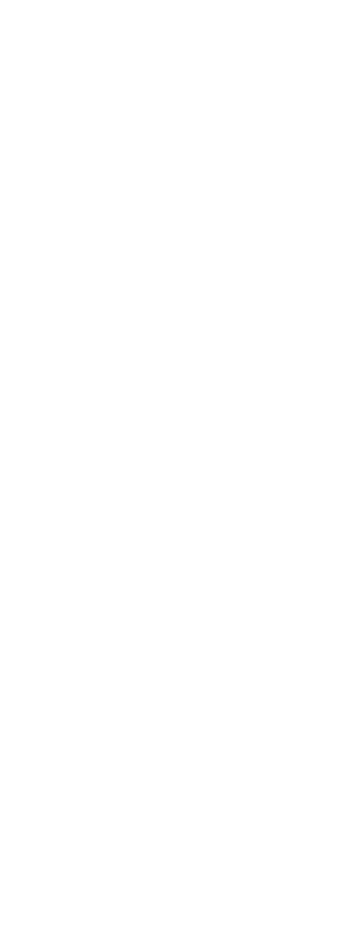$$
T_{\mathrm{f}}=100 \mathrm{~K}
$$ 


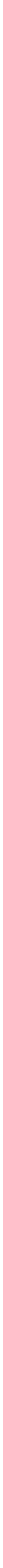

Figure 1c

\section{Figure 1c}

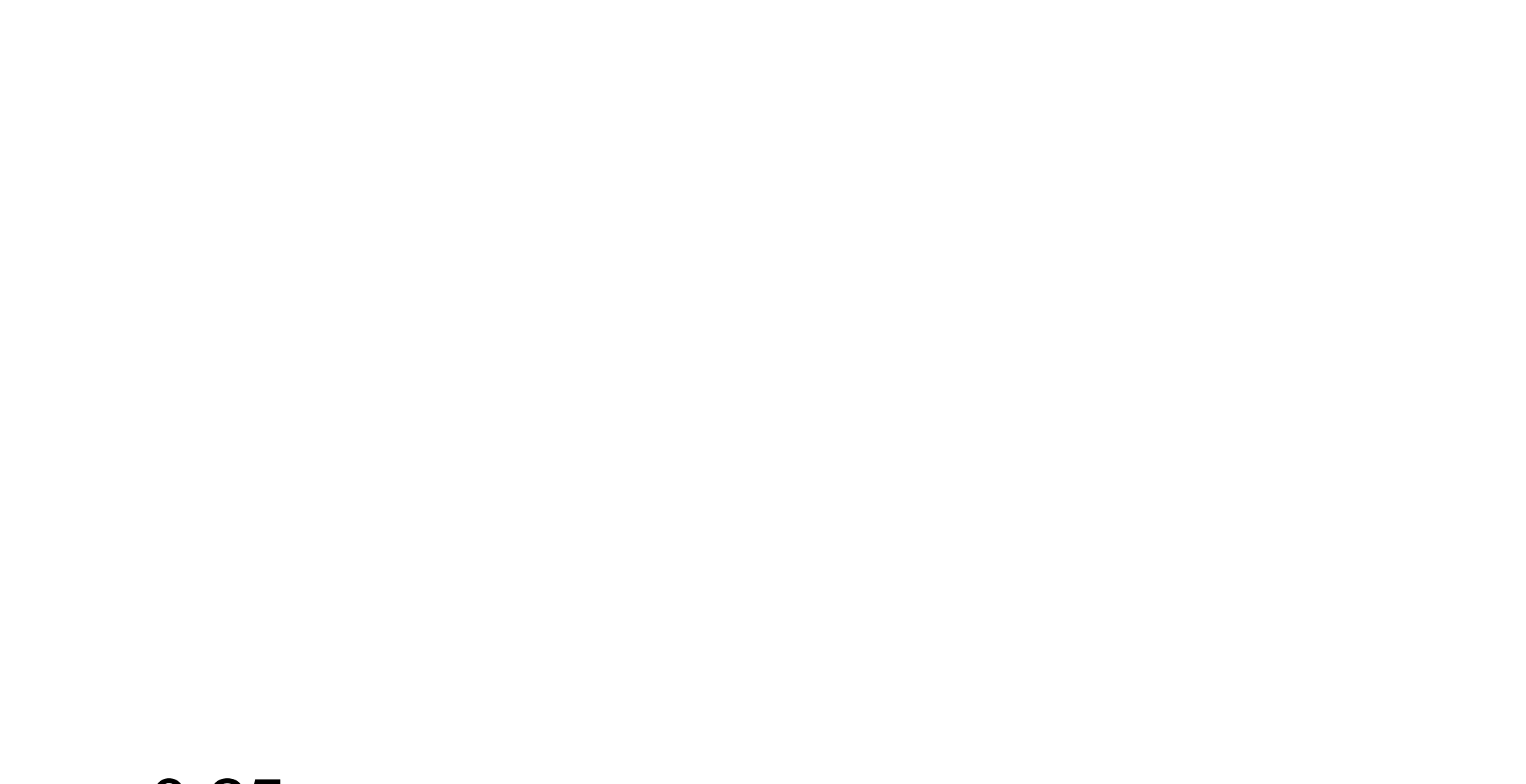


Figure 1d
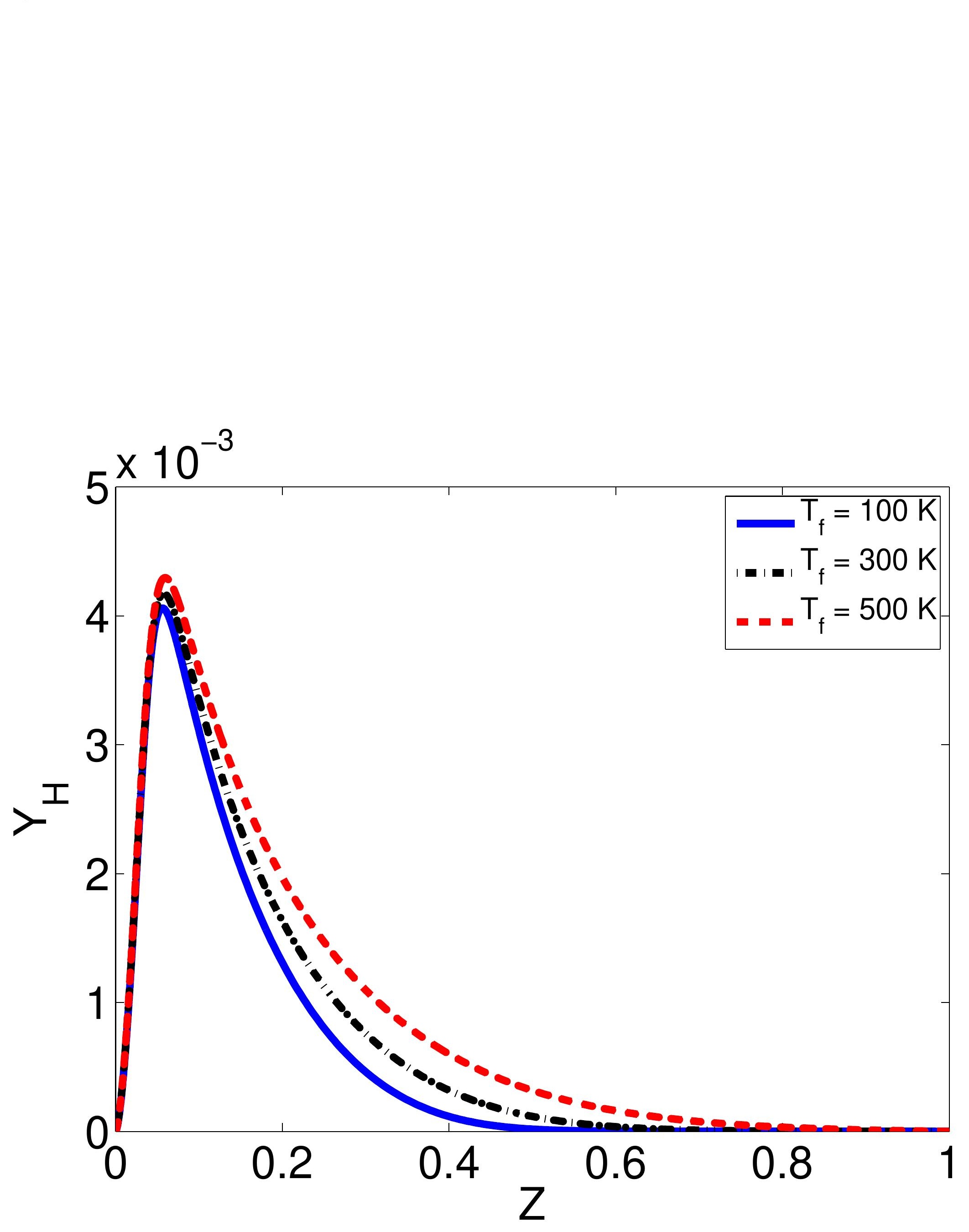

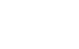




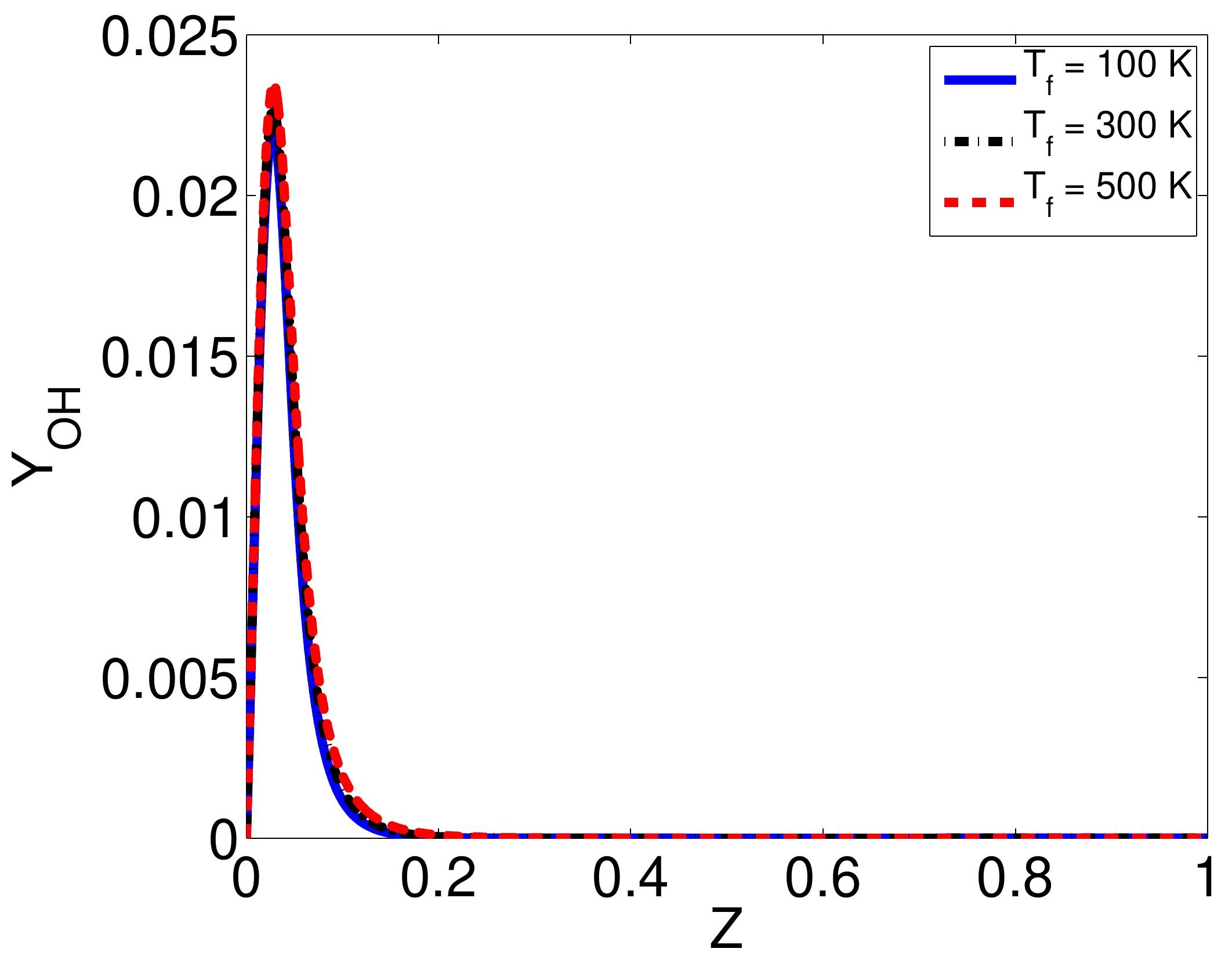

Figure 1e 


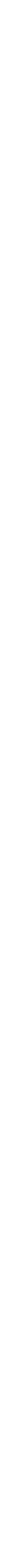

Figure $1 f$

|
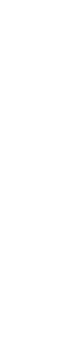

.

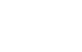

.

.

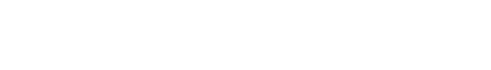




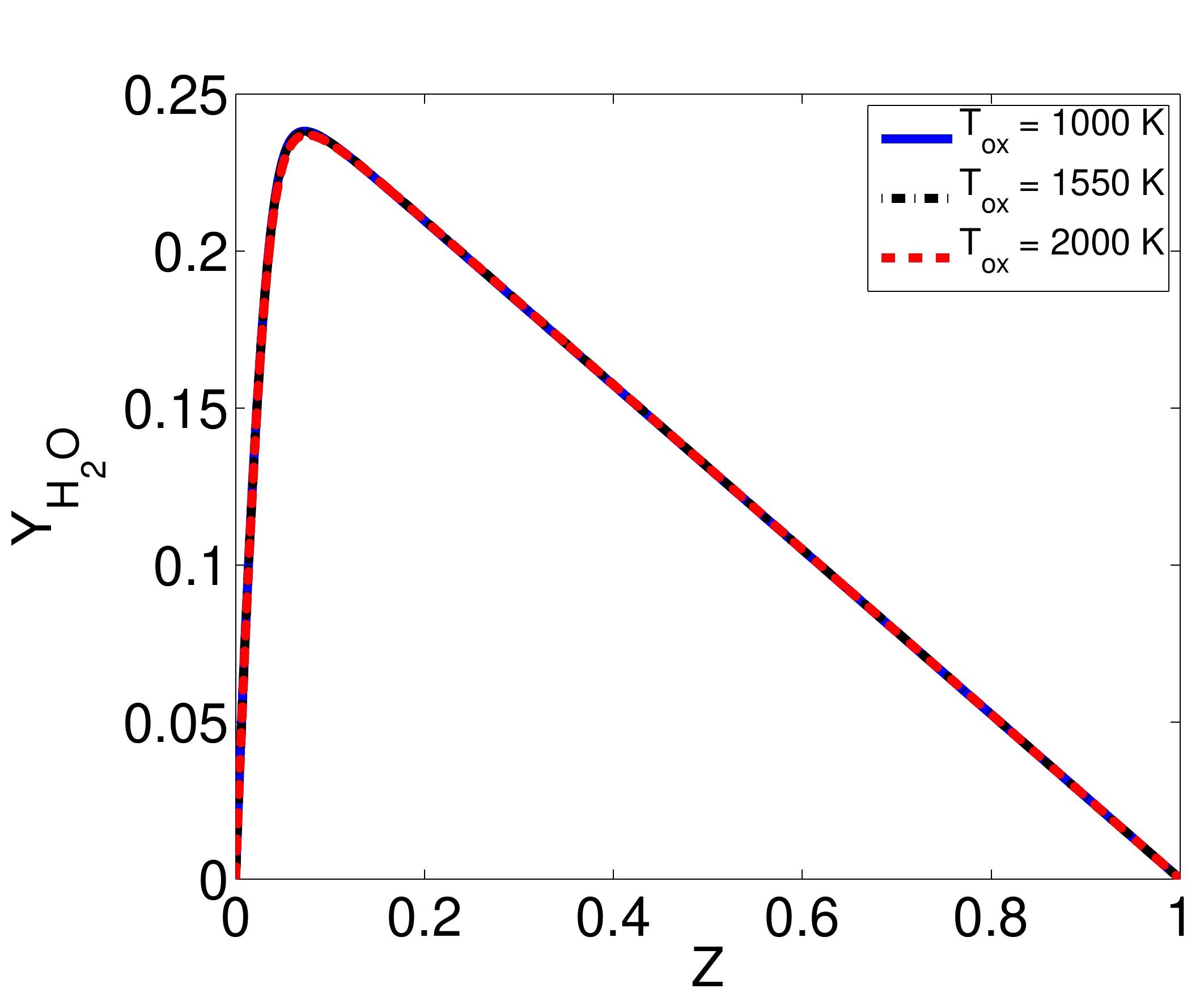

Figure 2a

.

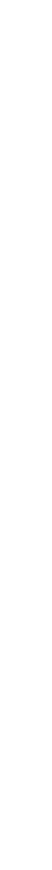

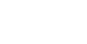

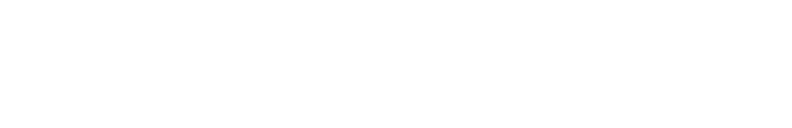

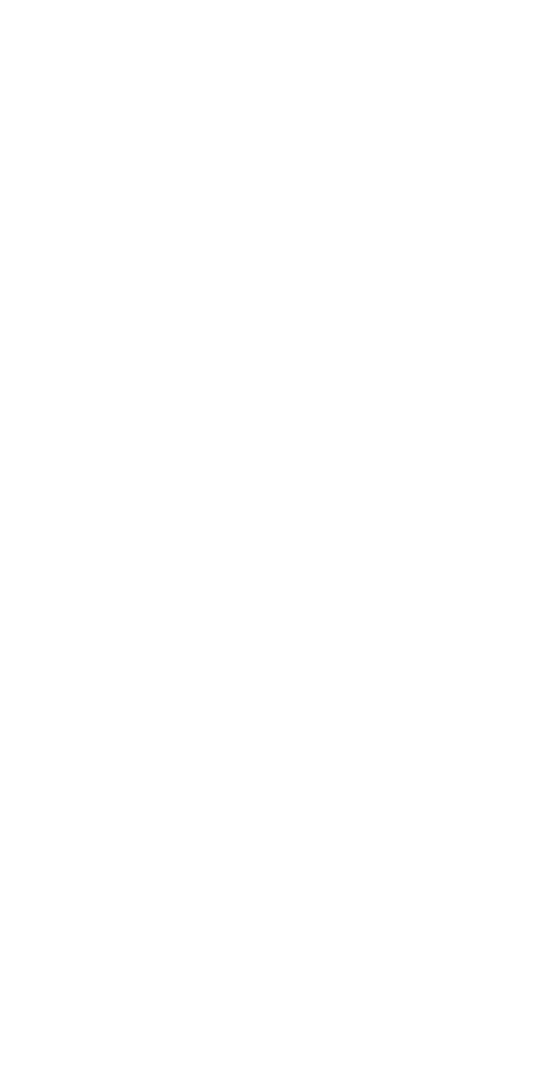


Figure 2b
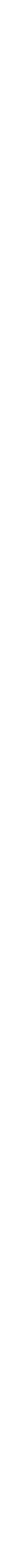

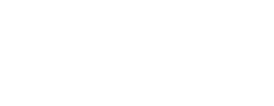

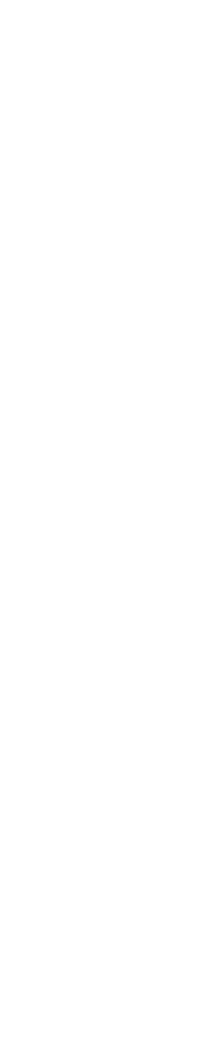




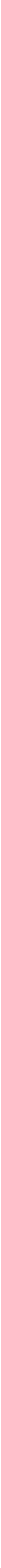

Figure 2c

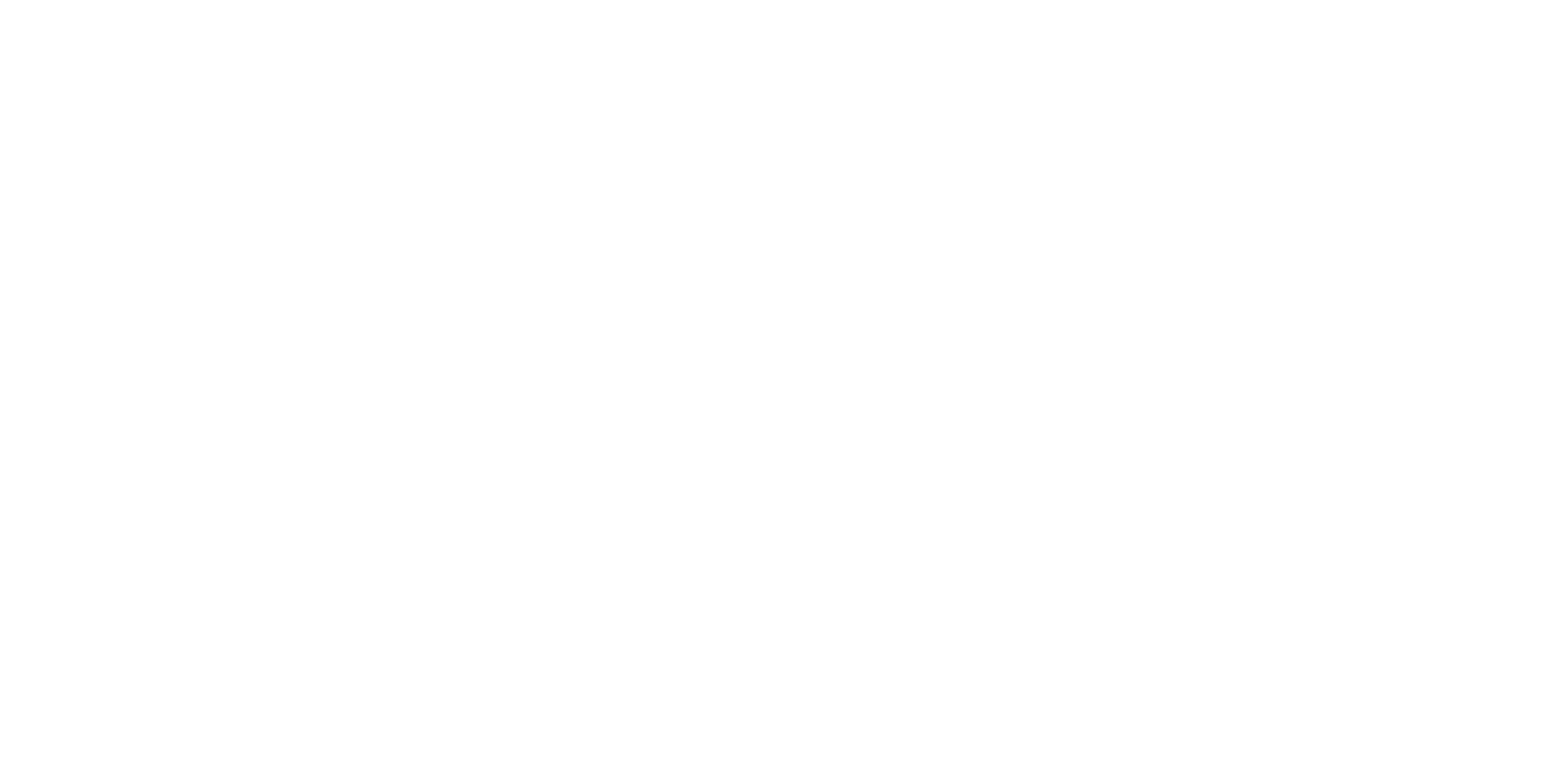






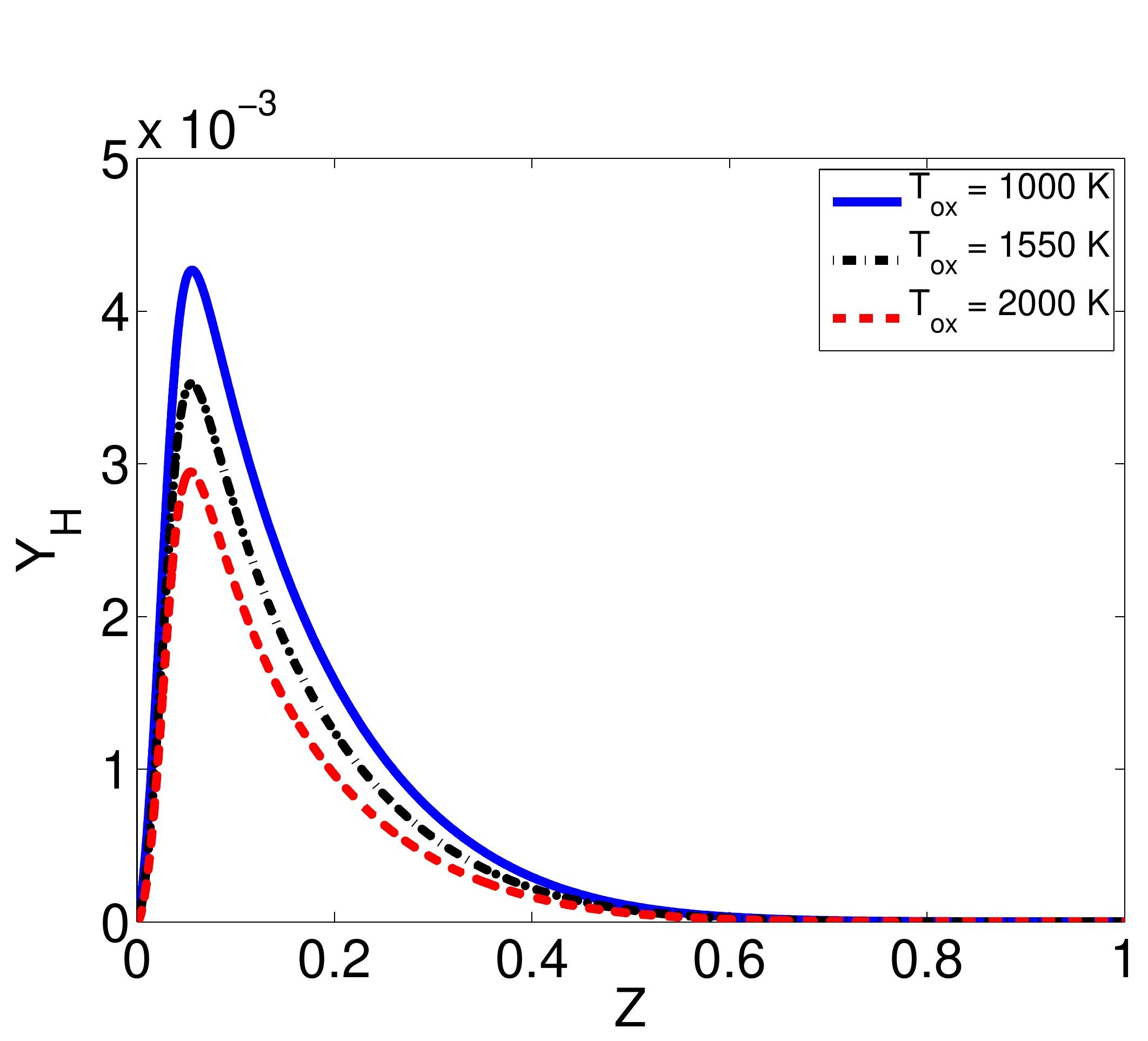

Figure 2d
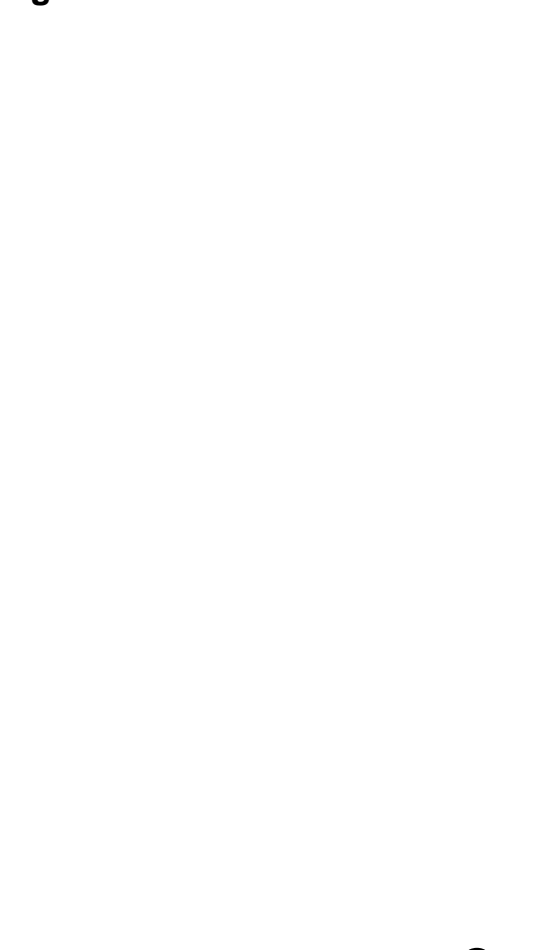

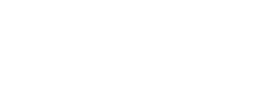

.

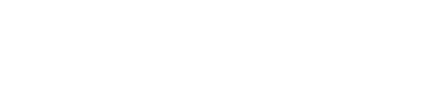




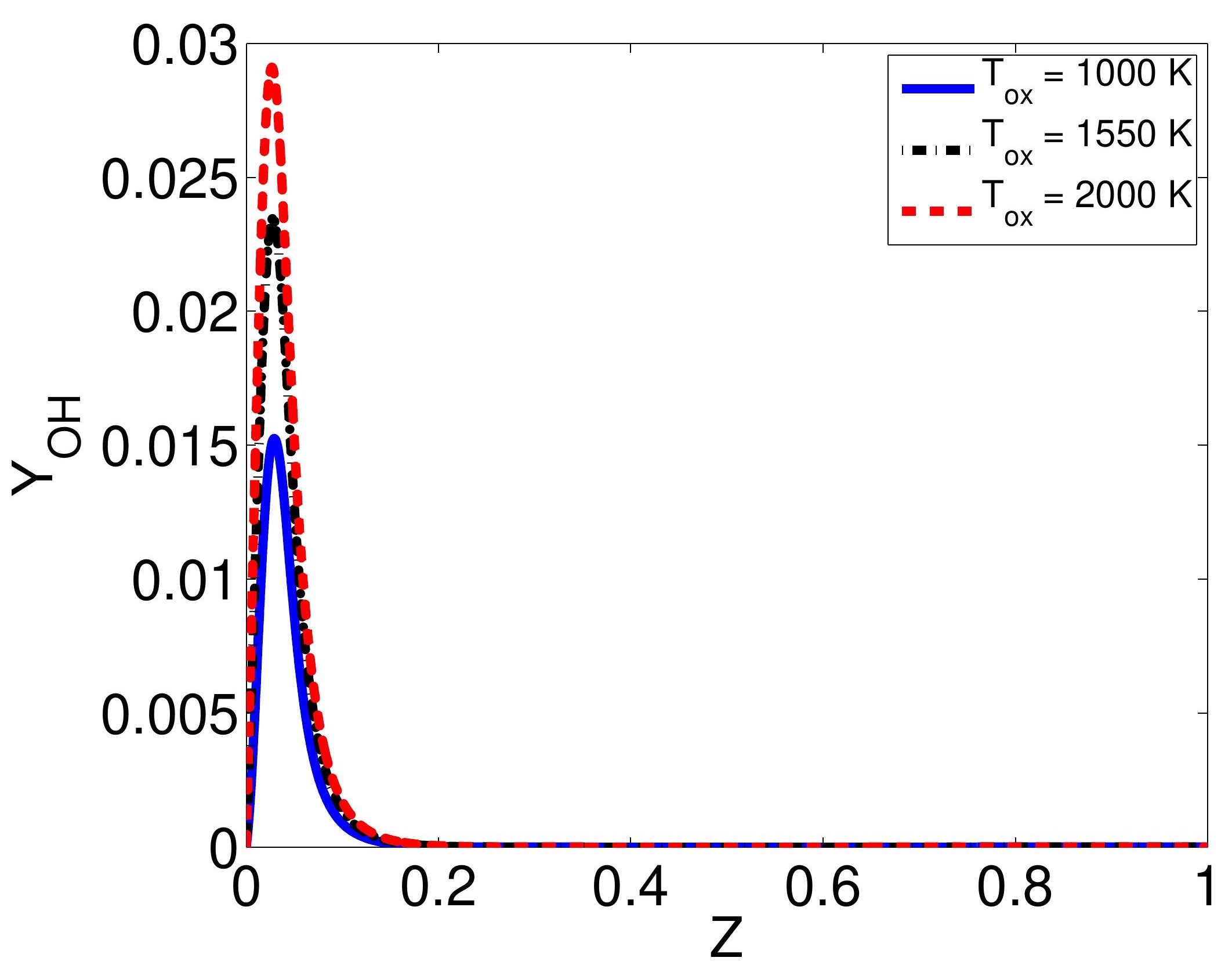

Figure 2e 


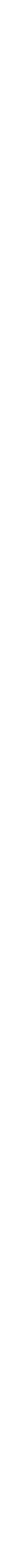

Figure $2 f$
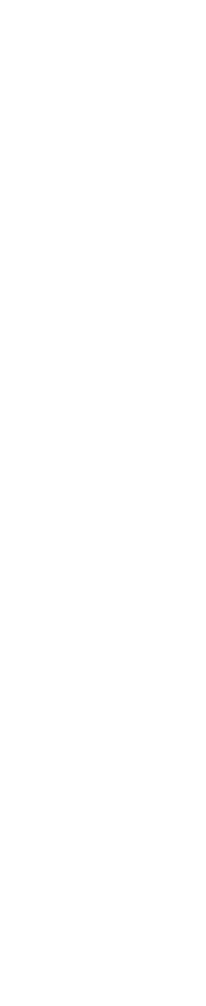

.

.

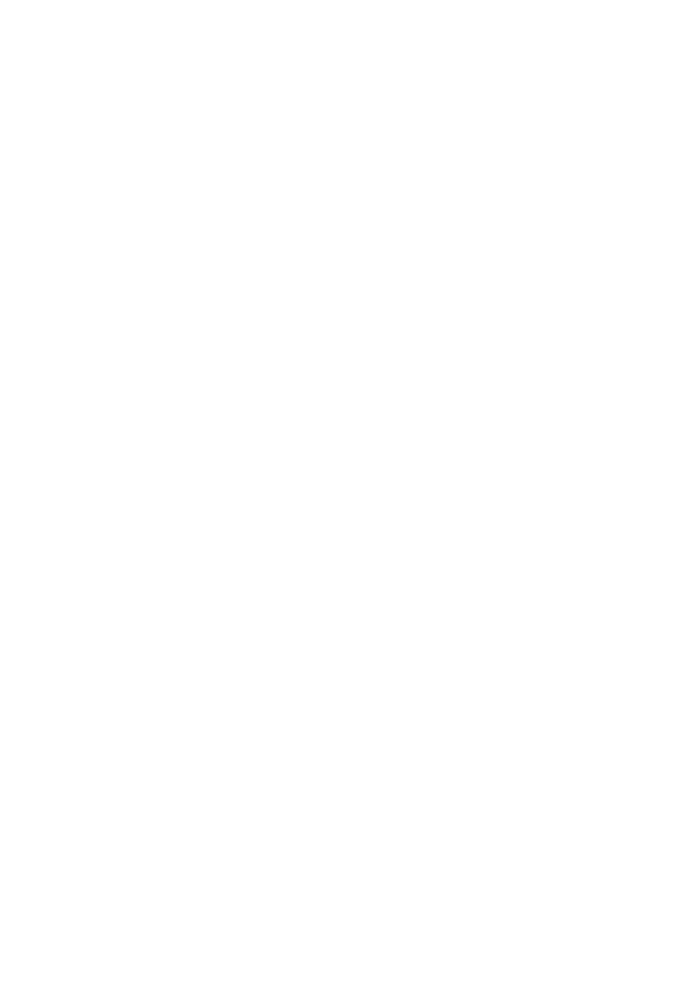




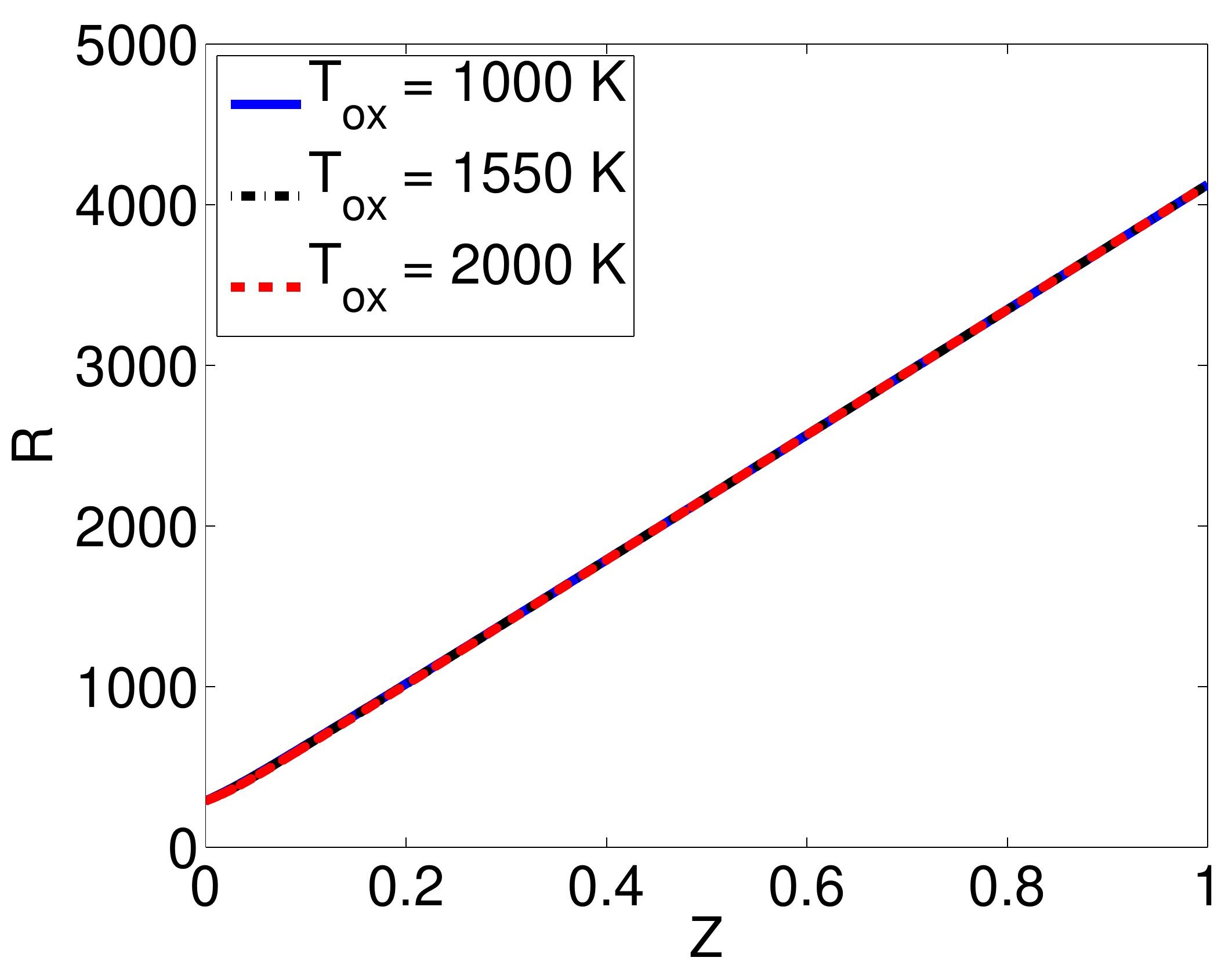

Figure 3

5000

$\mathrm{T}_{\mathrm{ox}}=1000 \mathrm{~K}$

$4000 \ldots \mathrm{T}_{\text {ox }}=1550 \mathrm{~K}$

. . . $\mathrm{T}_{\text {ox }}=2000 \mathrm{~K}$

3000 
Figure $4 a$

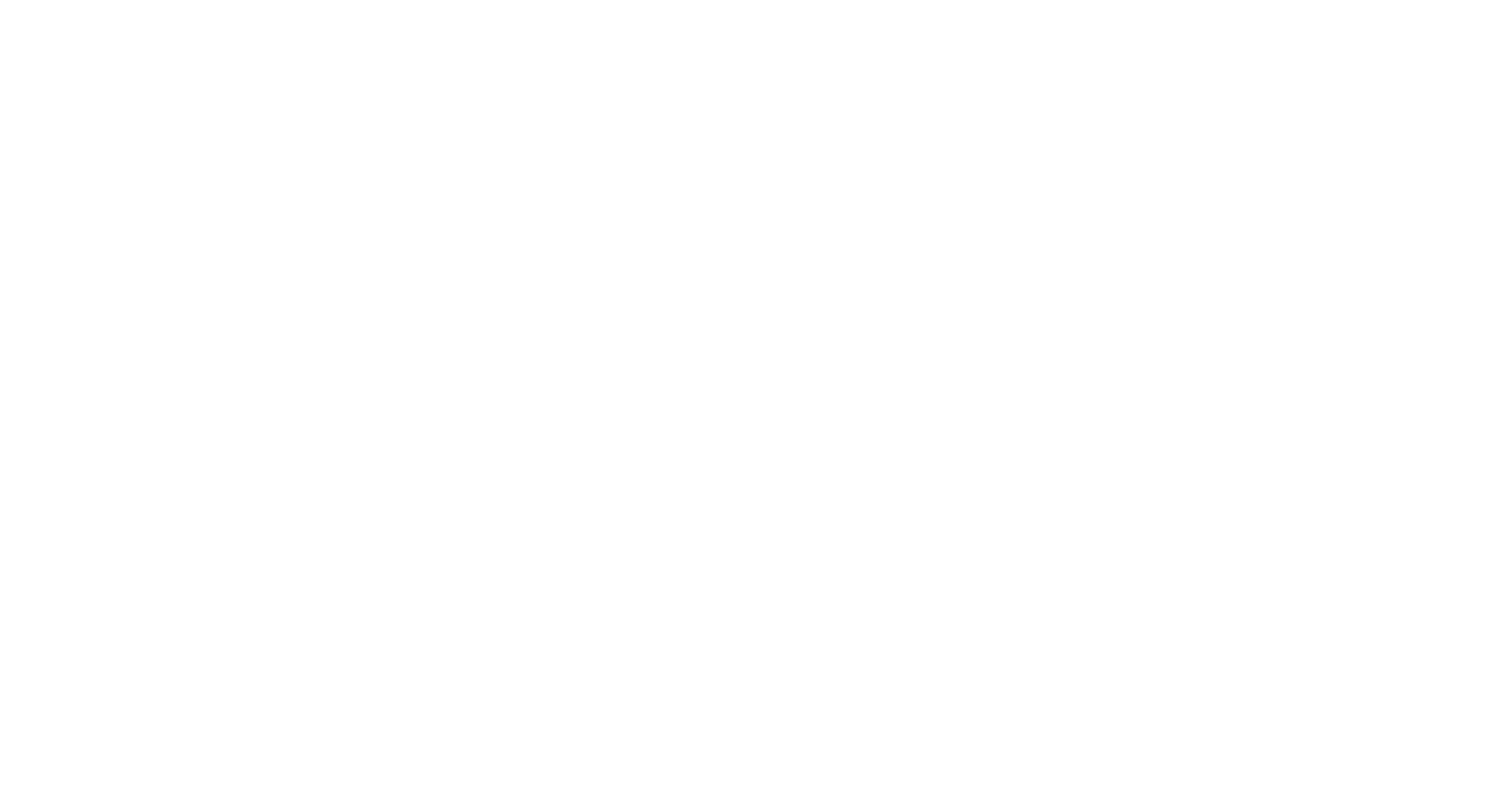

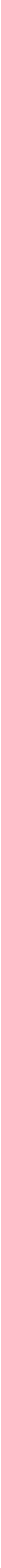

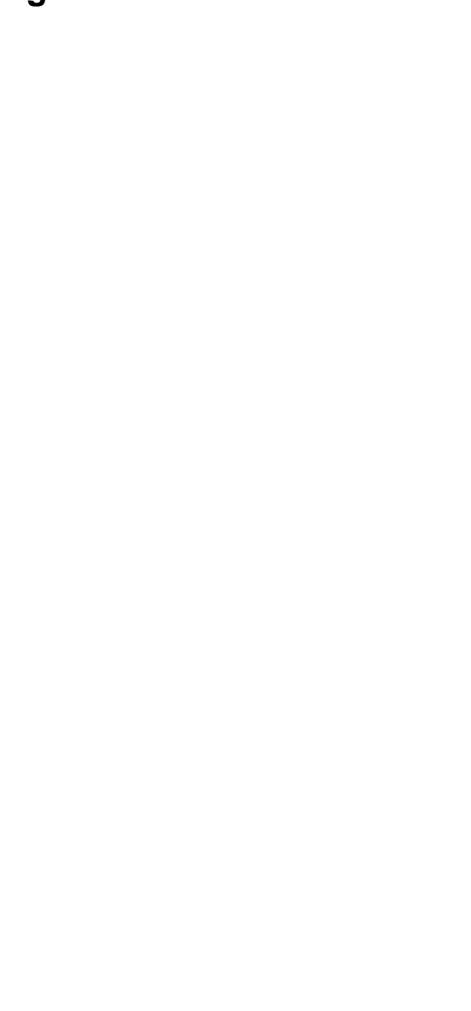




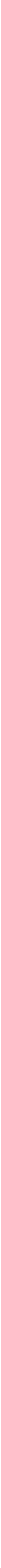

Figure $4 b$ 


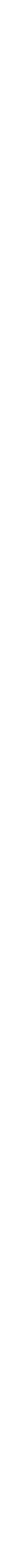

Figure 4c
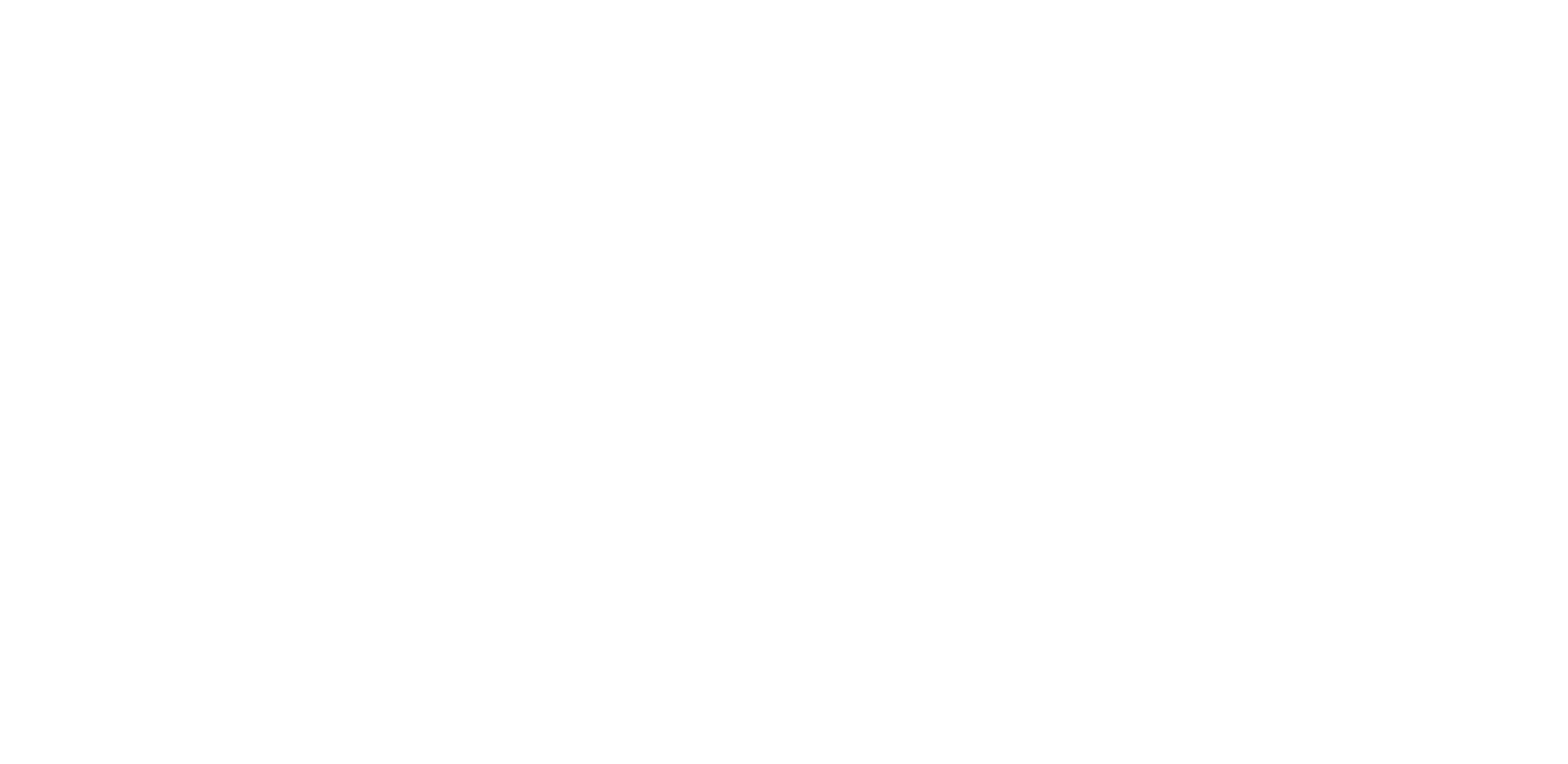


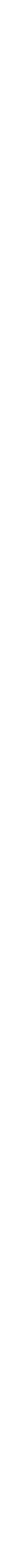

Figure 4d

.
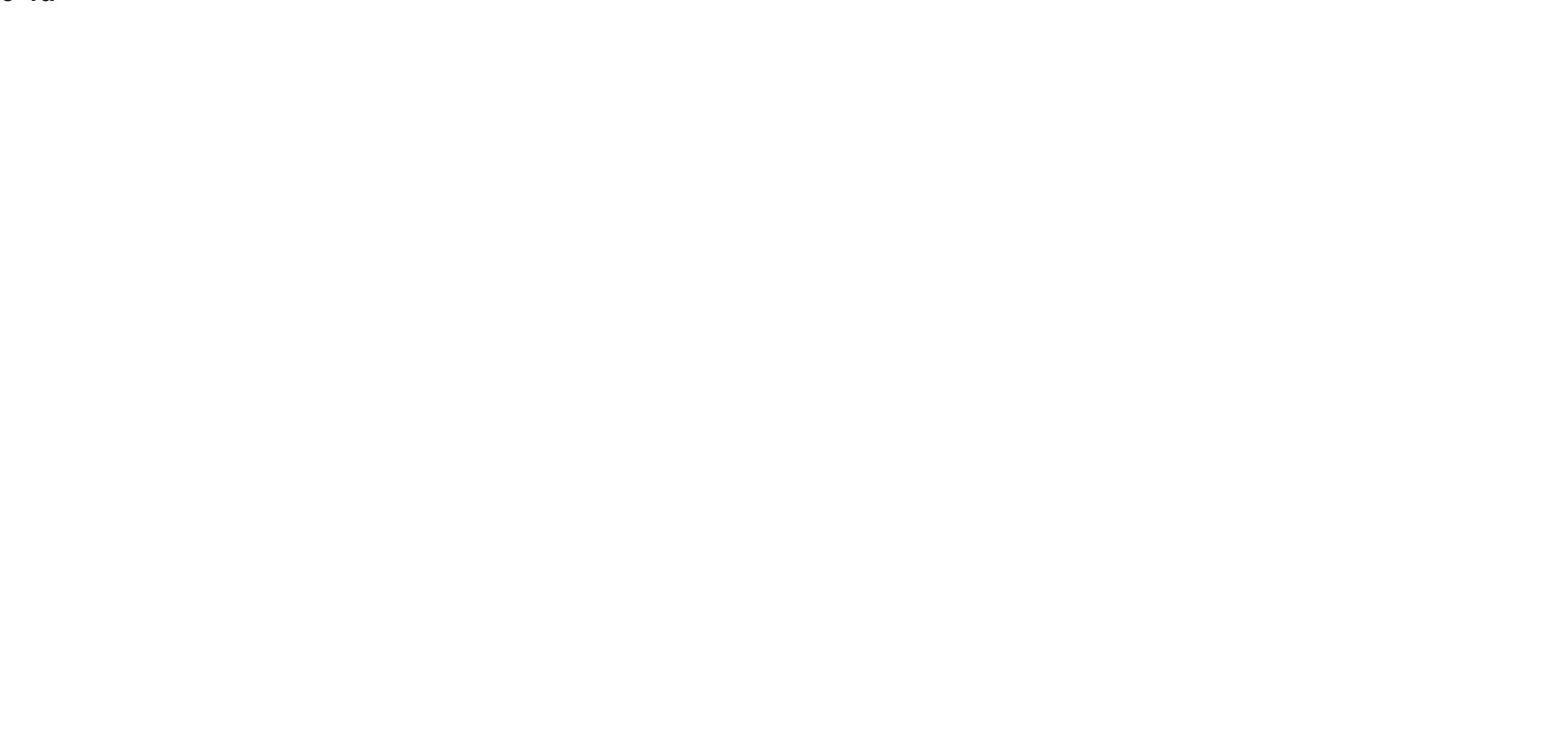
Figure $4 e$



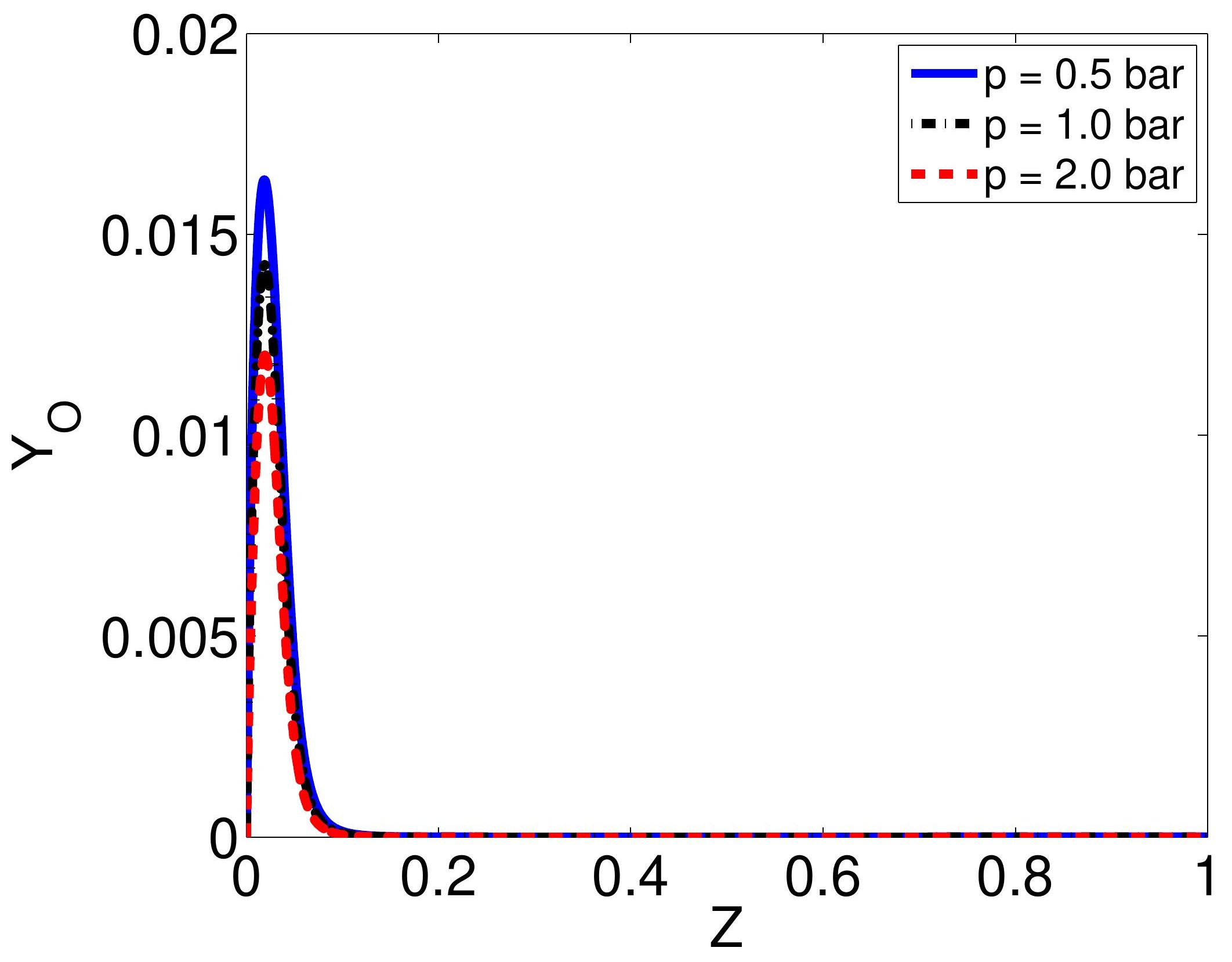

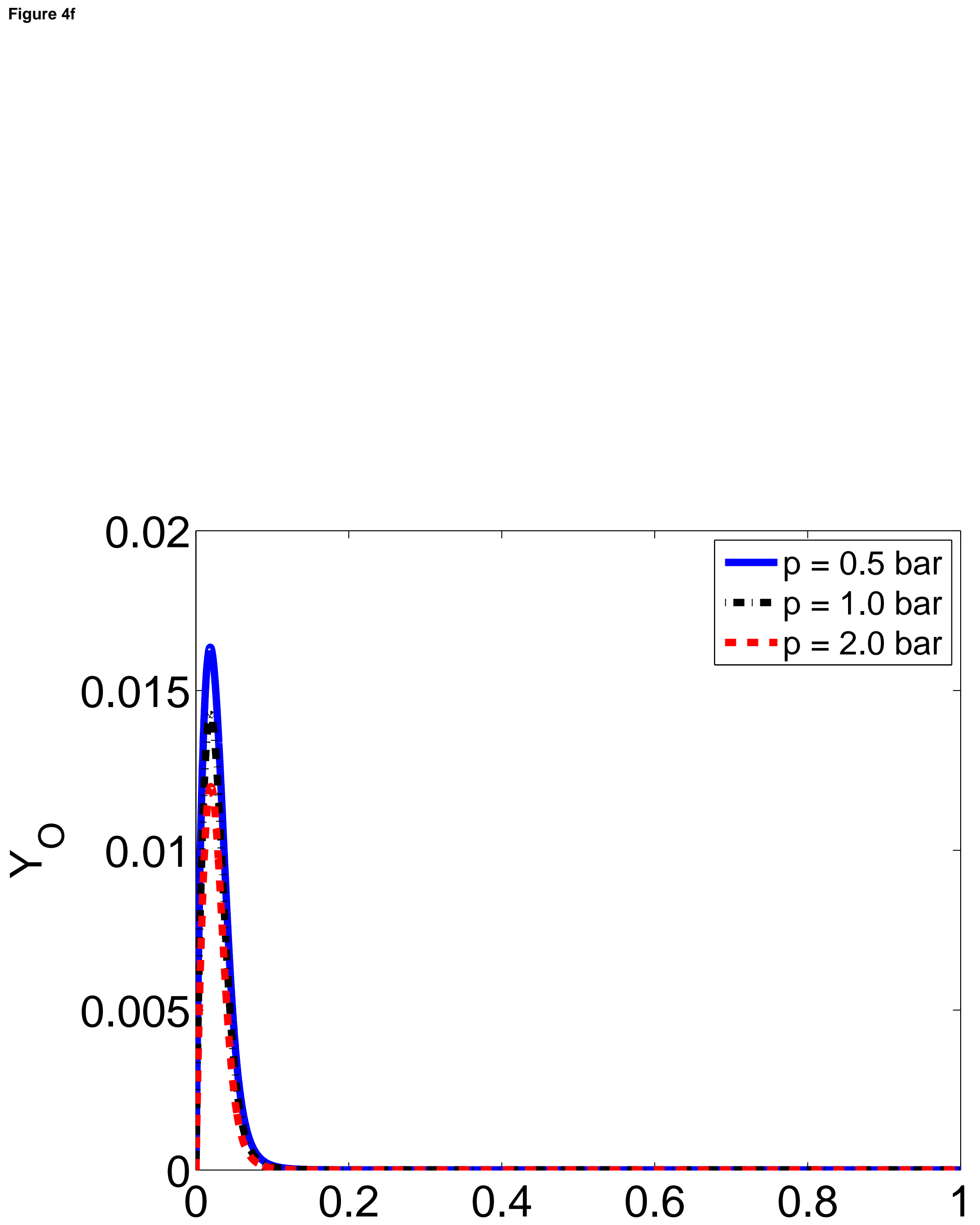

$$
\begin{array}{r}
-p=0.5 \mathrm{bar} \\
-p=1.0 \mathrm{bar} \\
-p=2.0 \mathrm{bar}
\end{array}
$$

0.02$$
\text { ( }
$$ 
Figure $5 b$



Thes

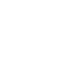



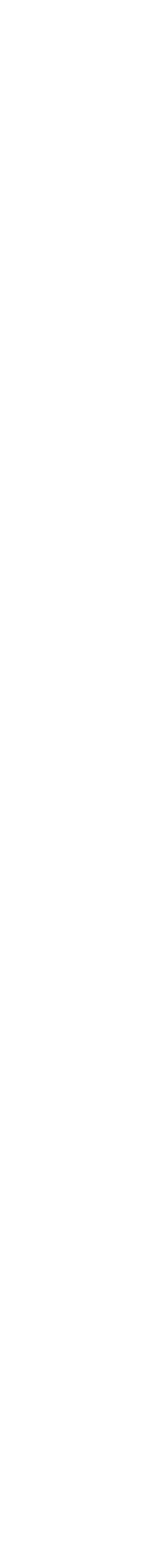

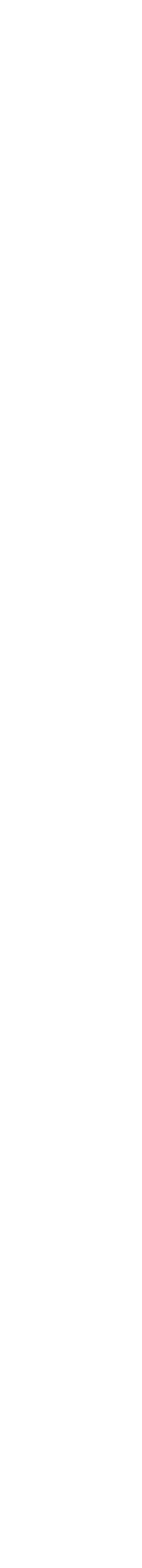

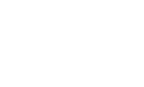


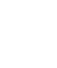

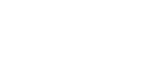






Figure 6 


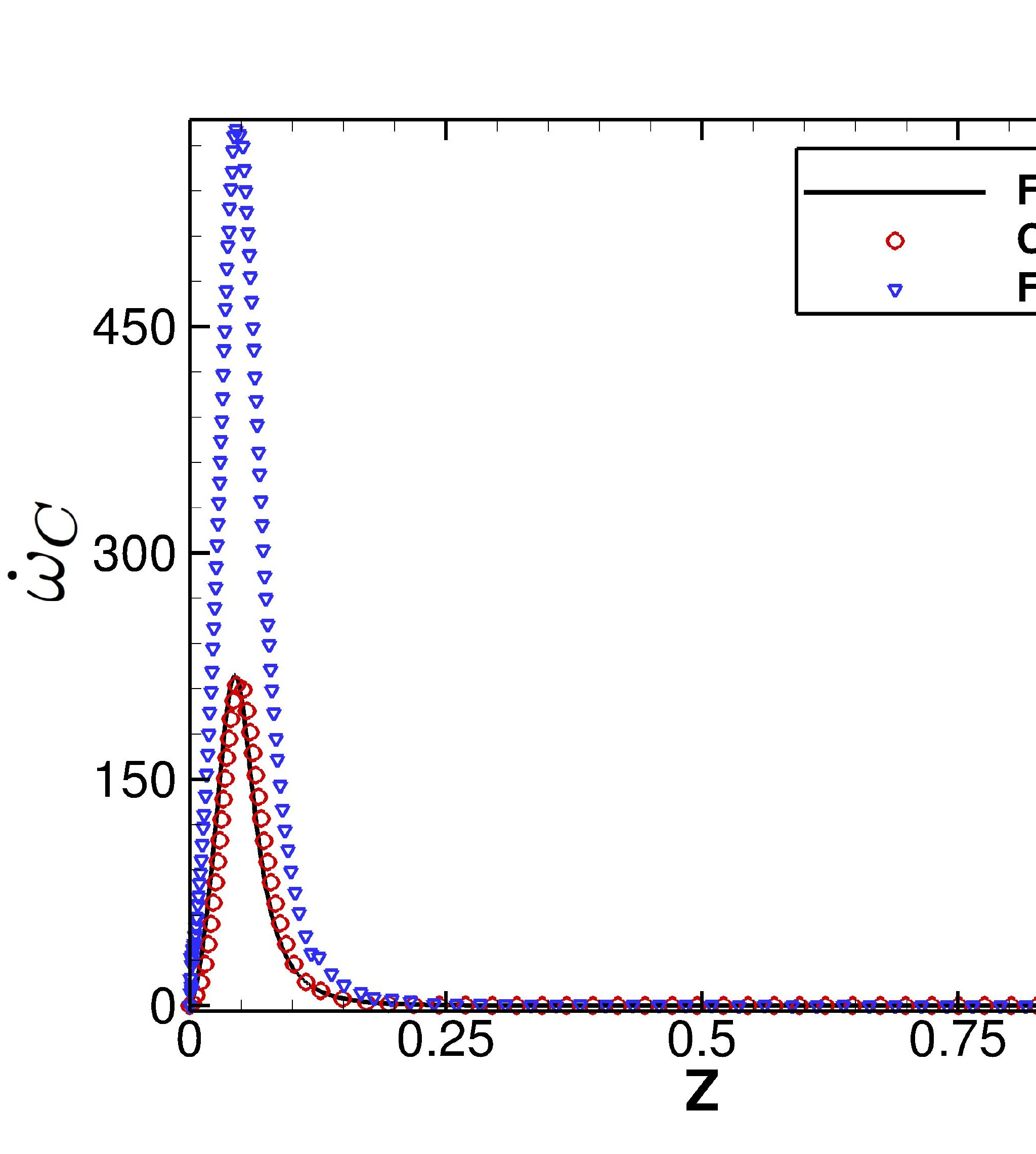

Figure 7

\section{Figure 7}




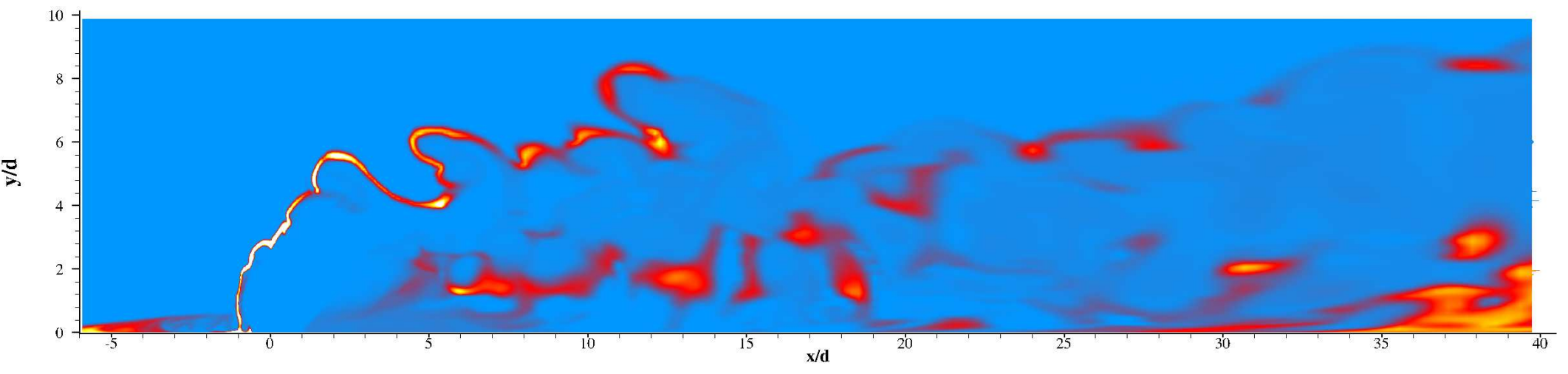




\section{Figure $8 b$}

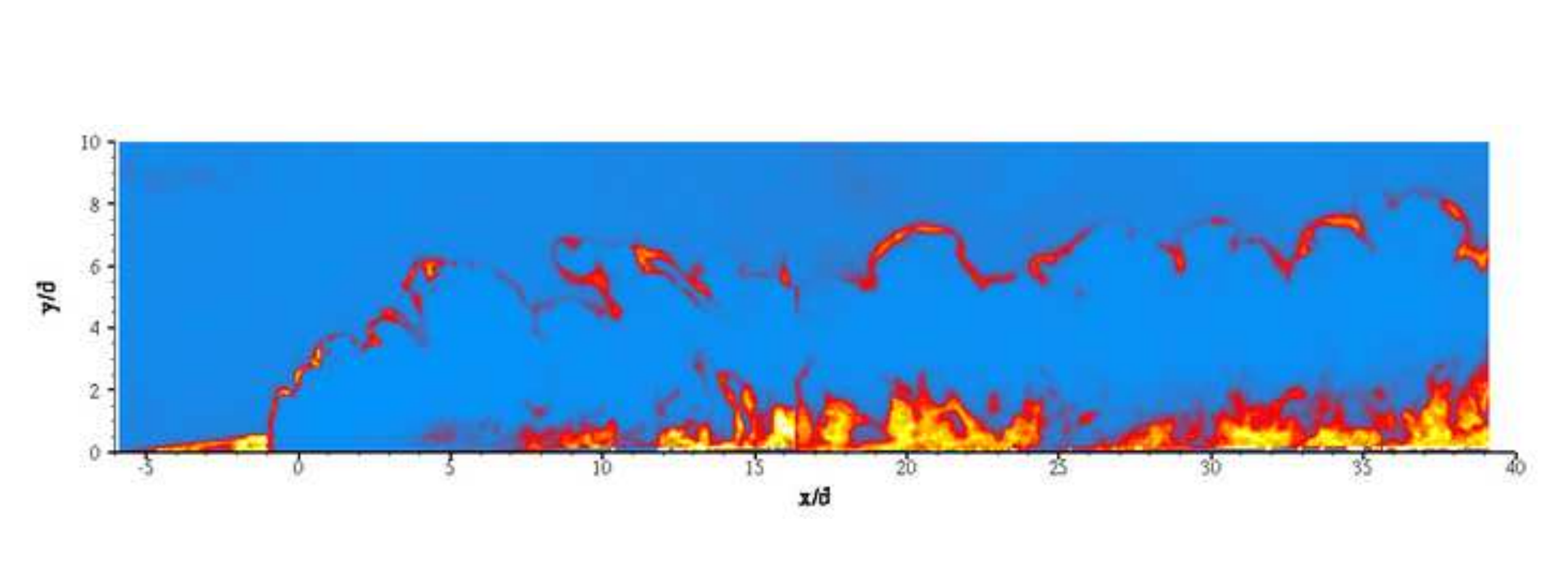



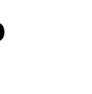

(

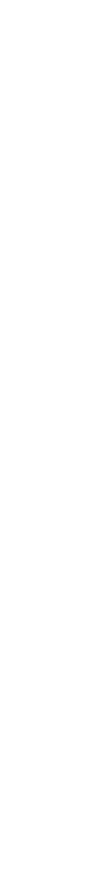

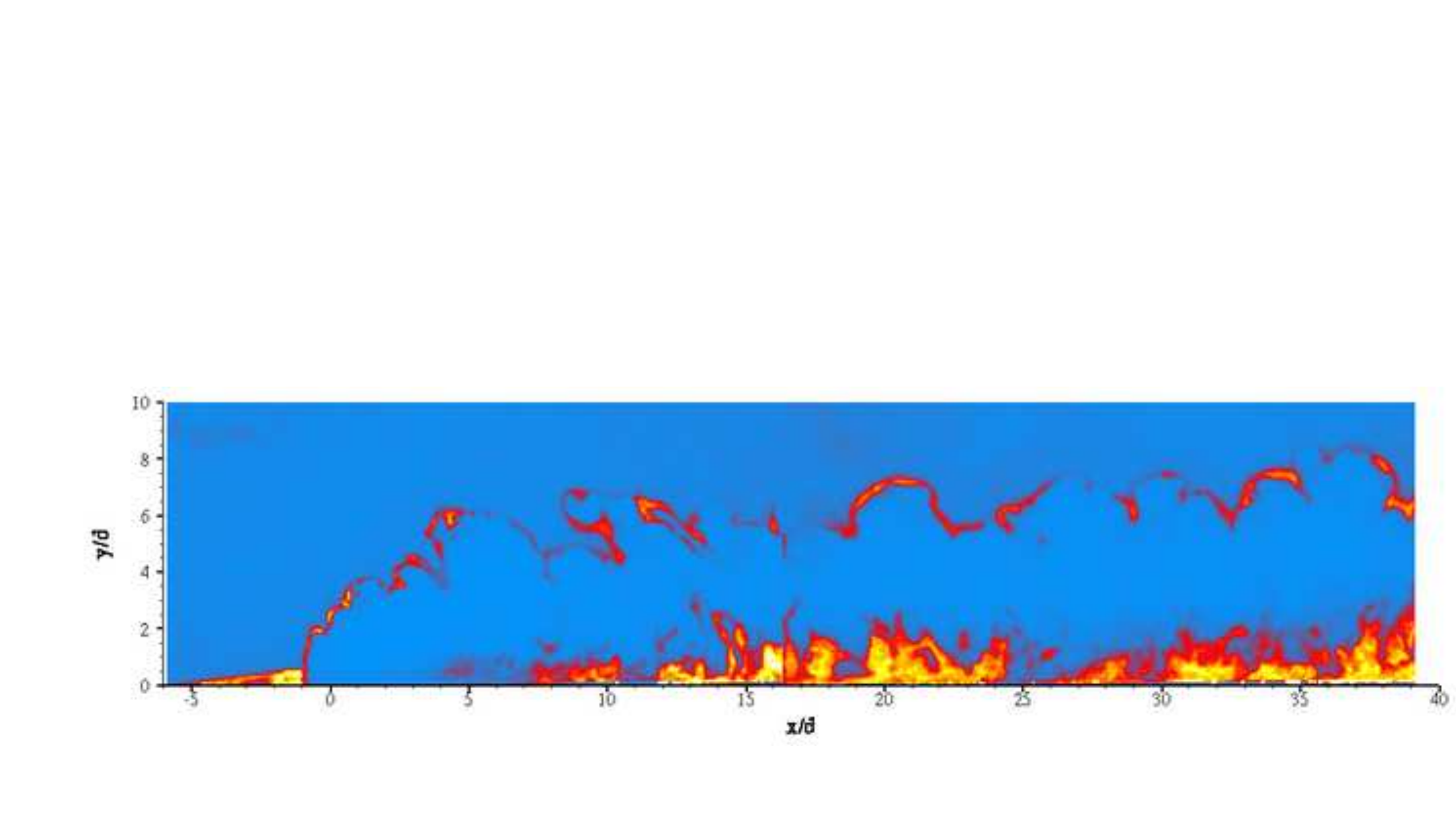


Figure 9

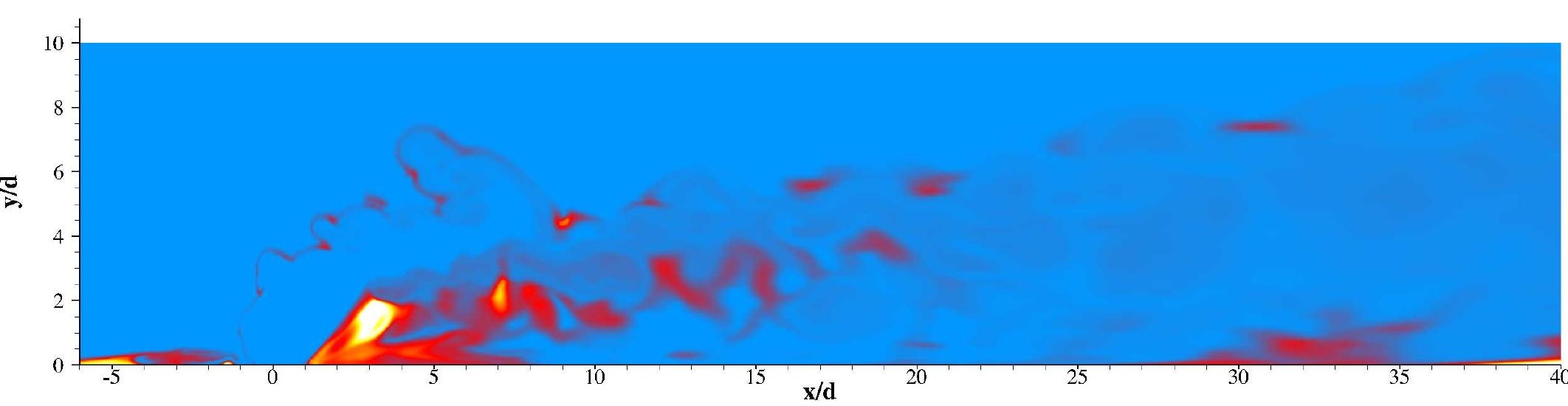




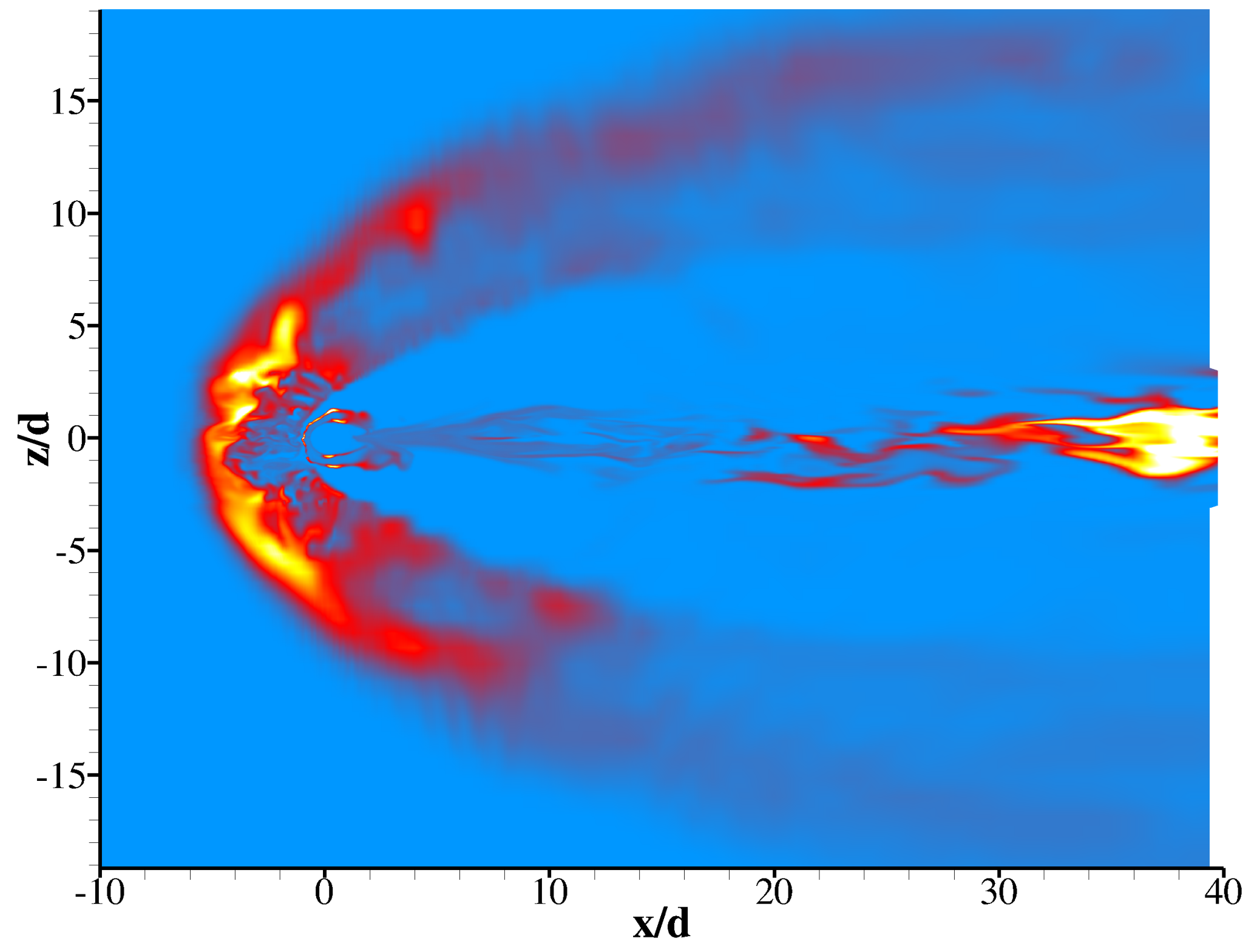




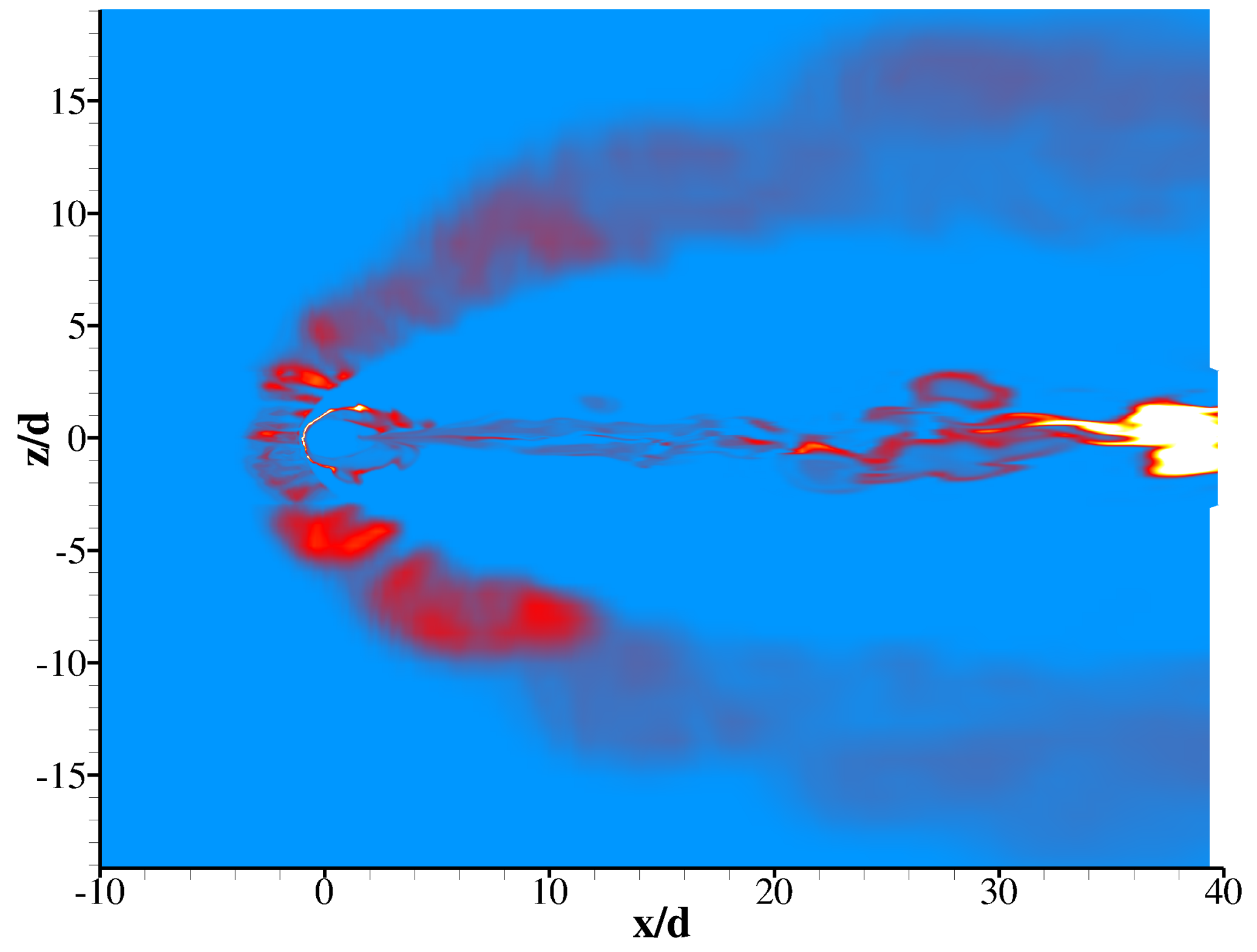







Figure 10d






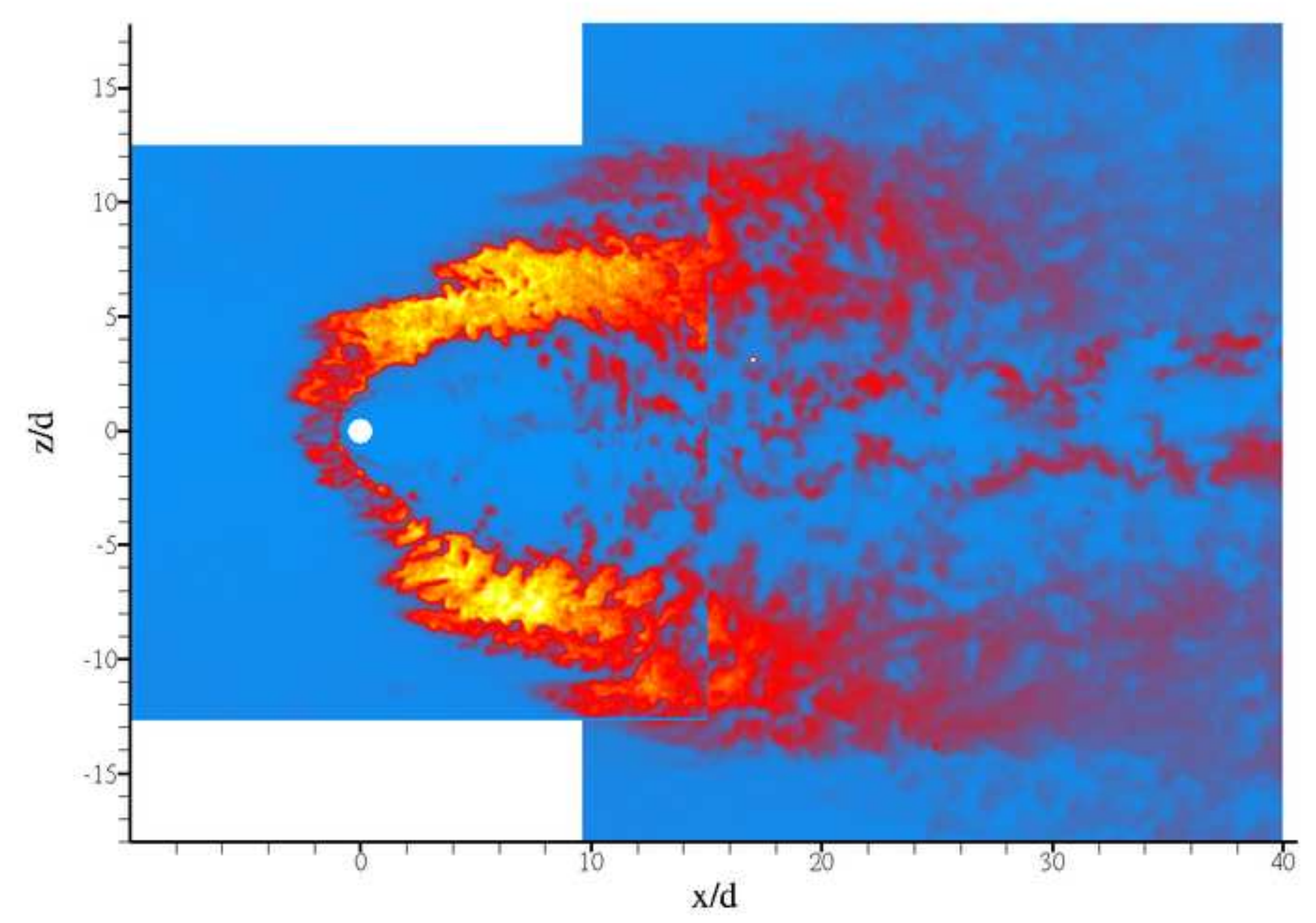




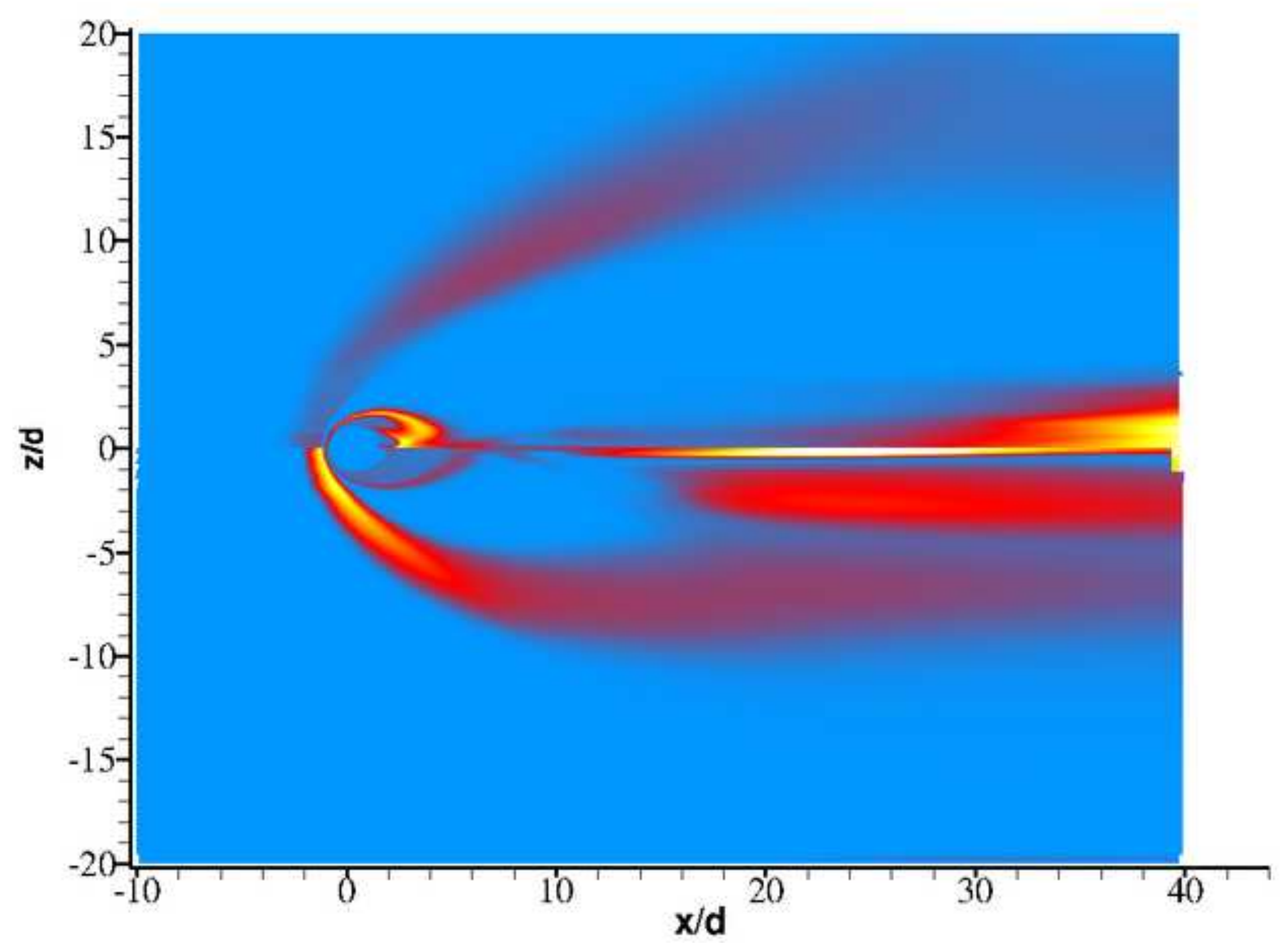




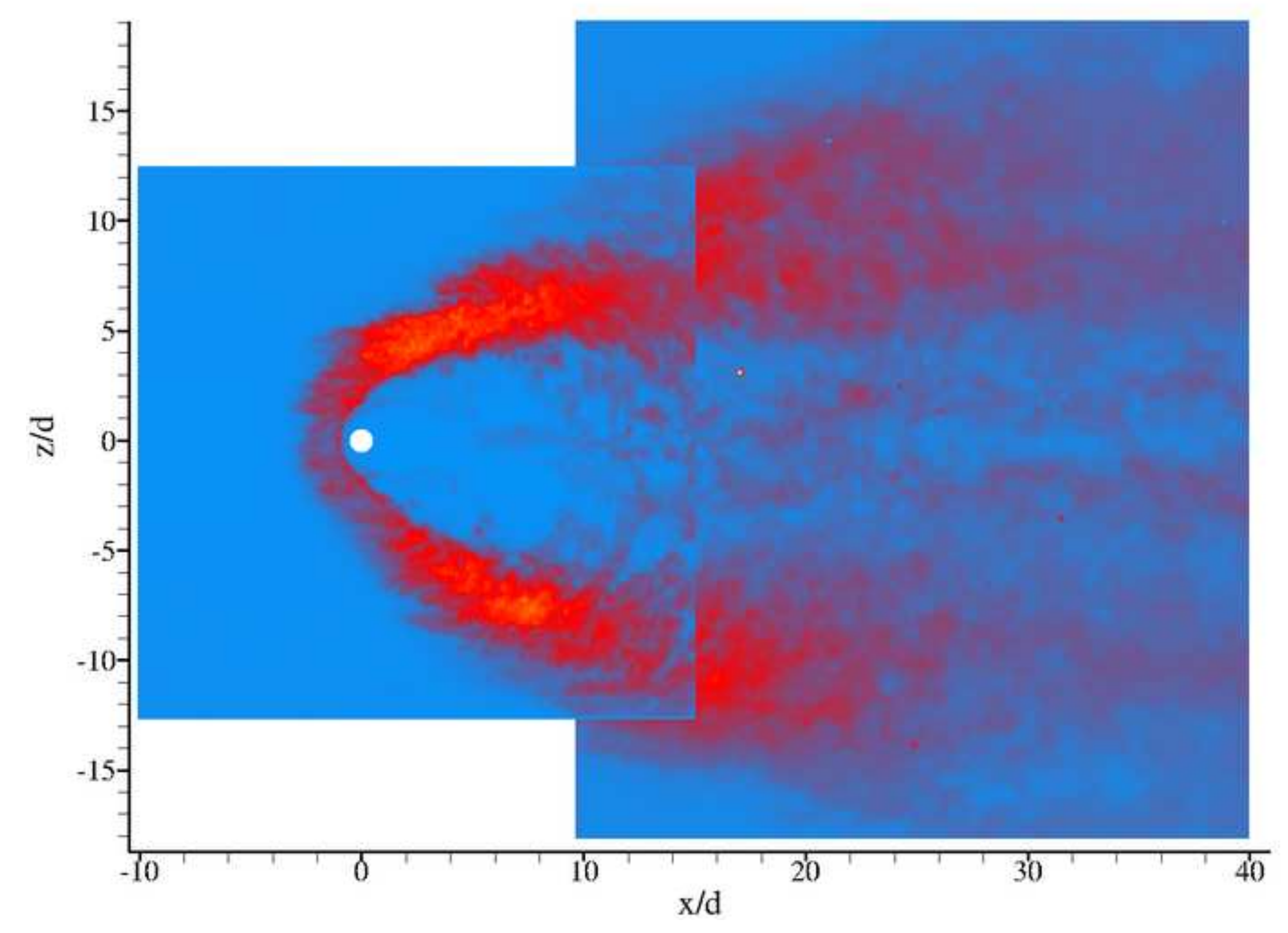


Figure 14a

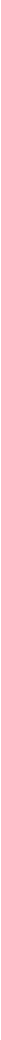

$\begin{array}{lll}\mathbf{x} / \mathbf{d}^{5} & 10 & 15\end{array}$






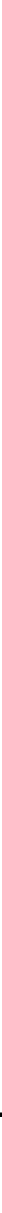

Figure $14 c$ 


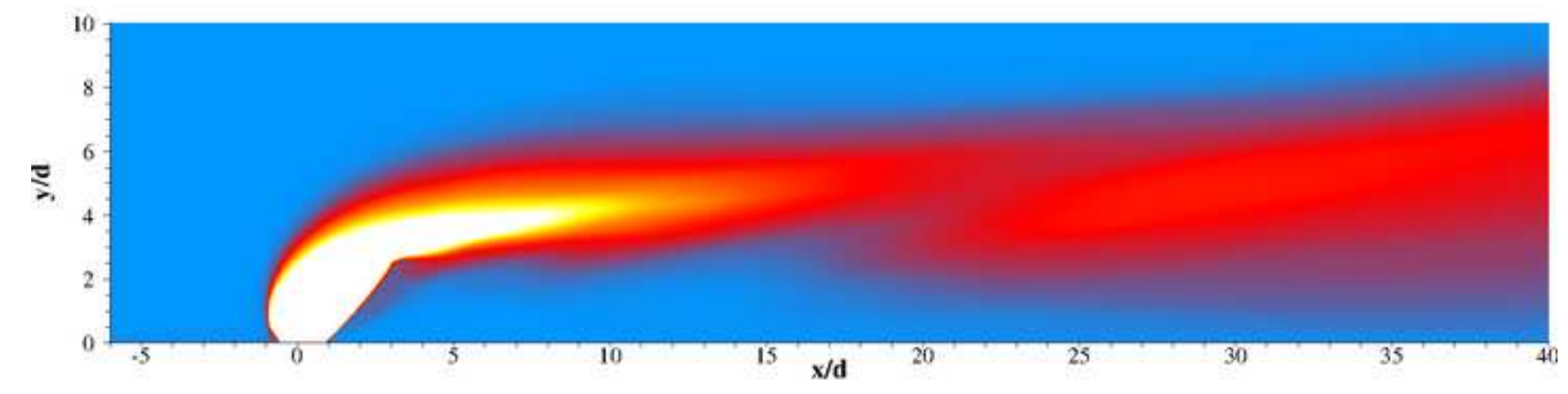

rare
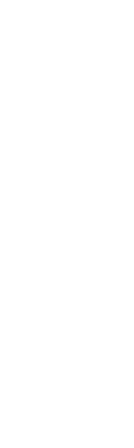
x/d 
Figure 16a

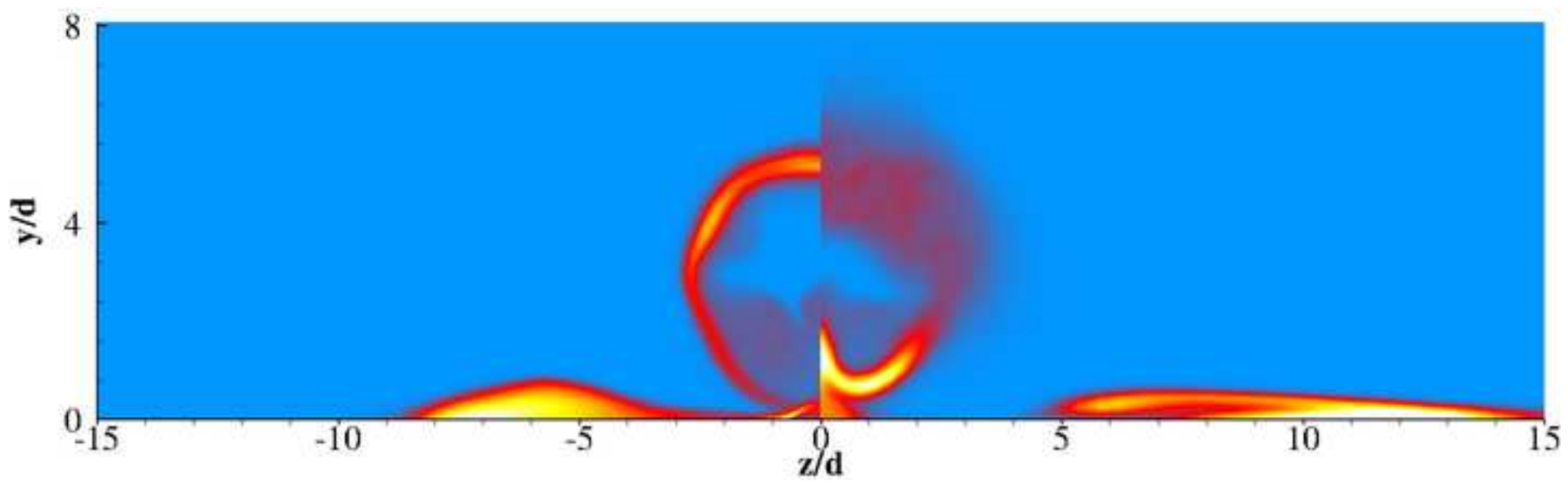




\section{Figure 16b}


.

. 
Figure $16 c$

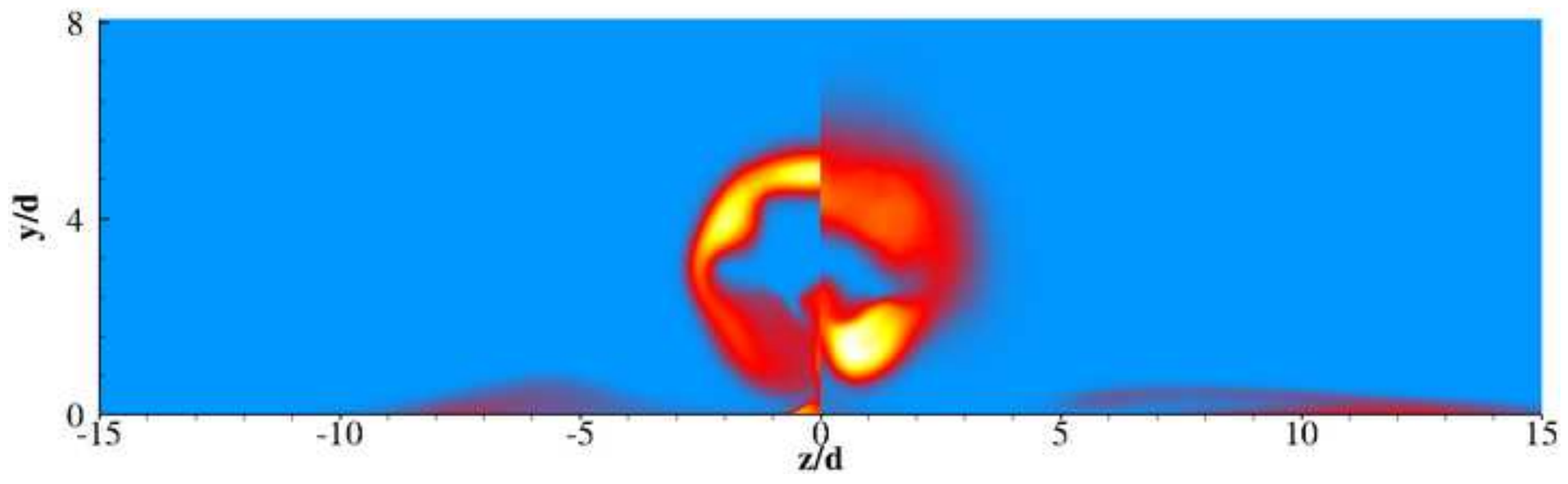


Figure 16d

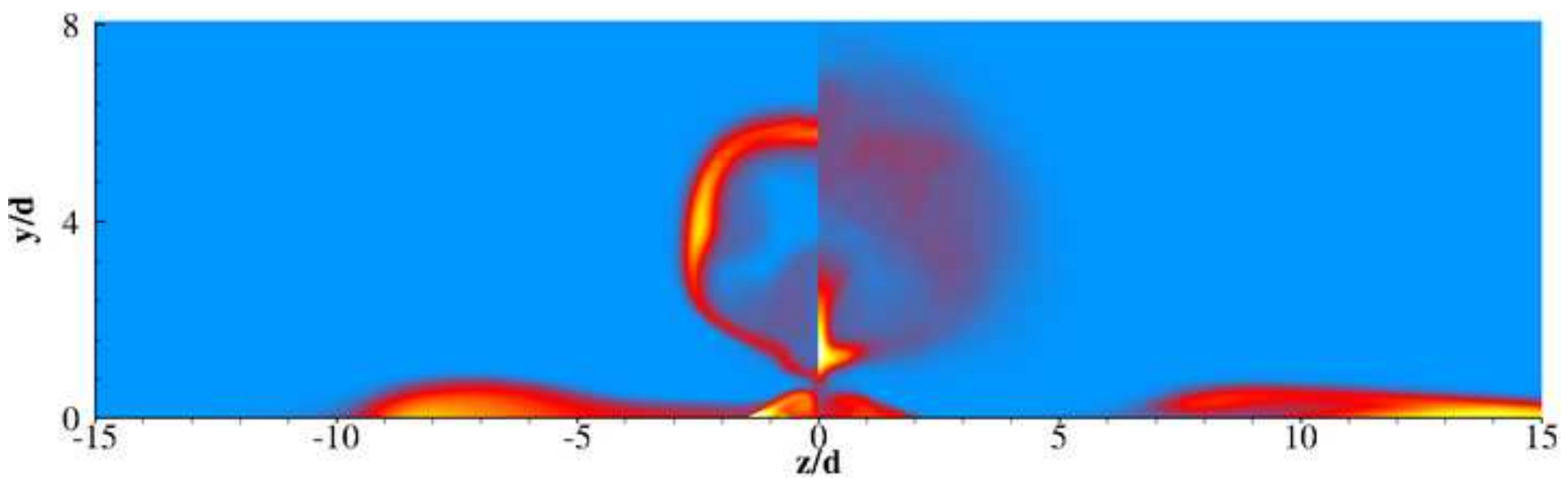




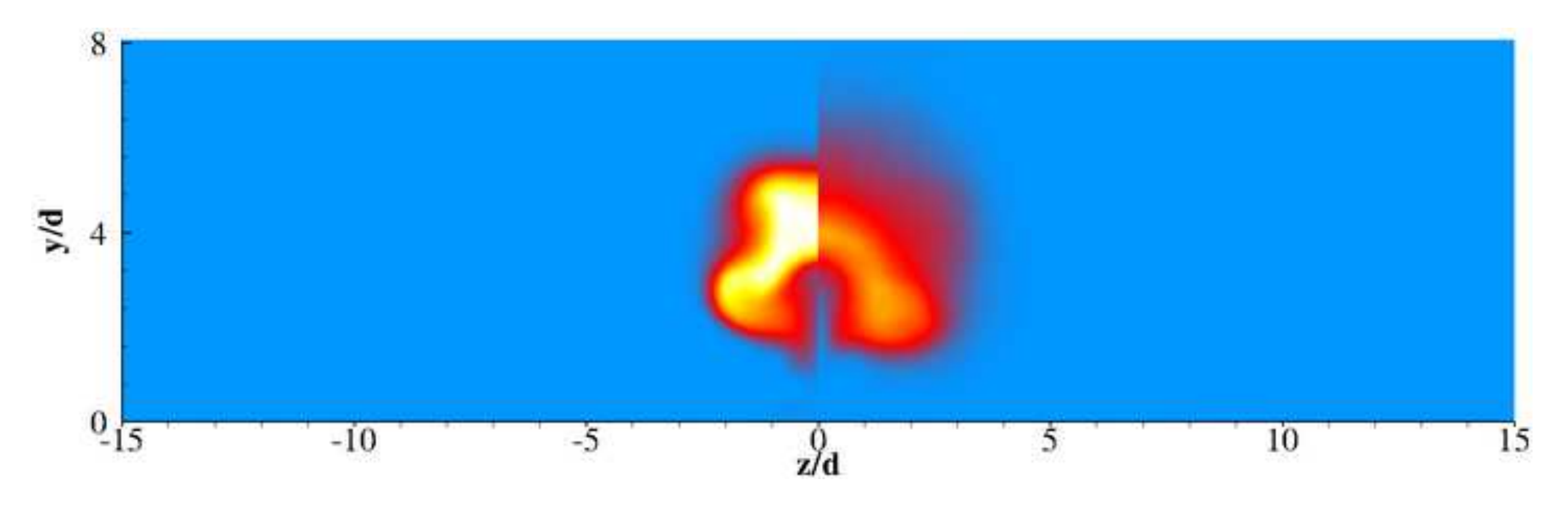

Fure

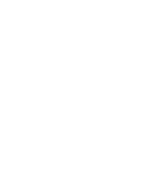

-

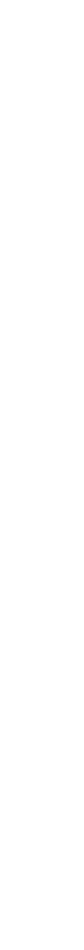




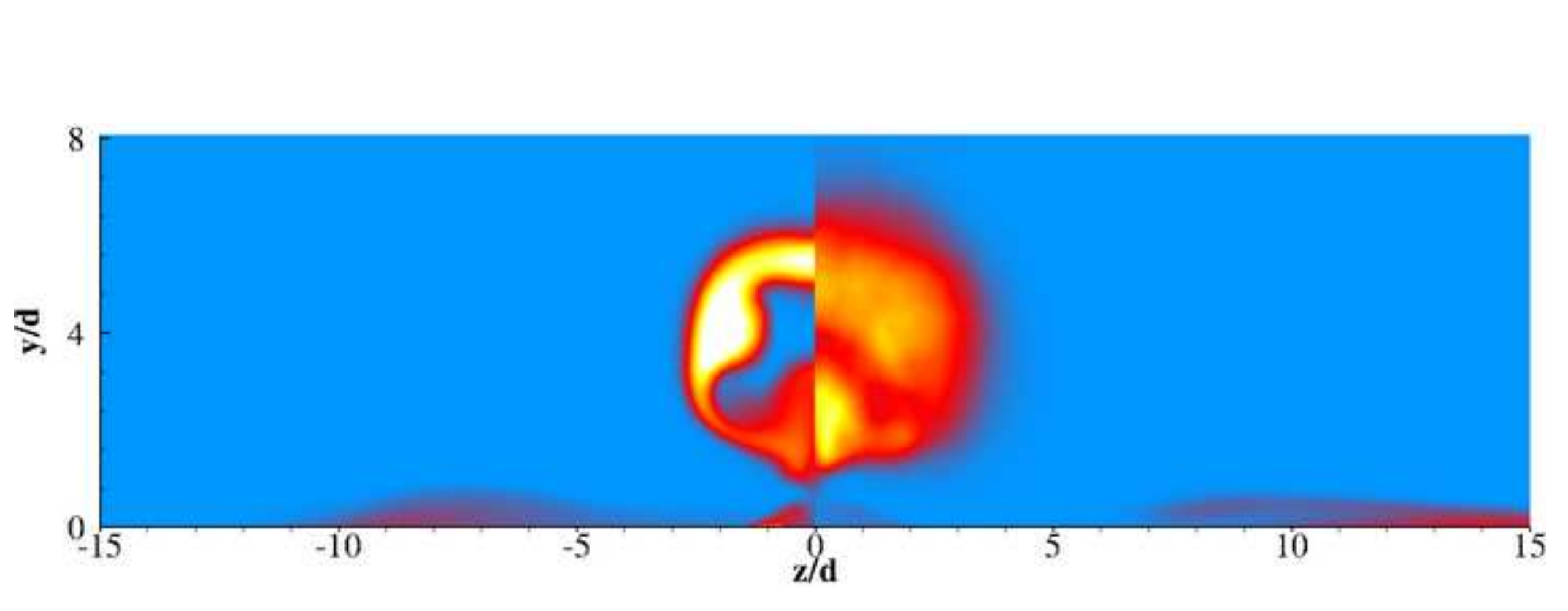

5
(




Figure $16 \mathrm{~g}$

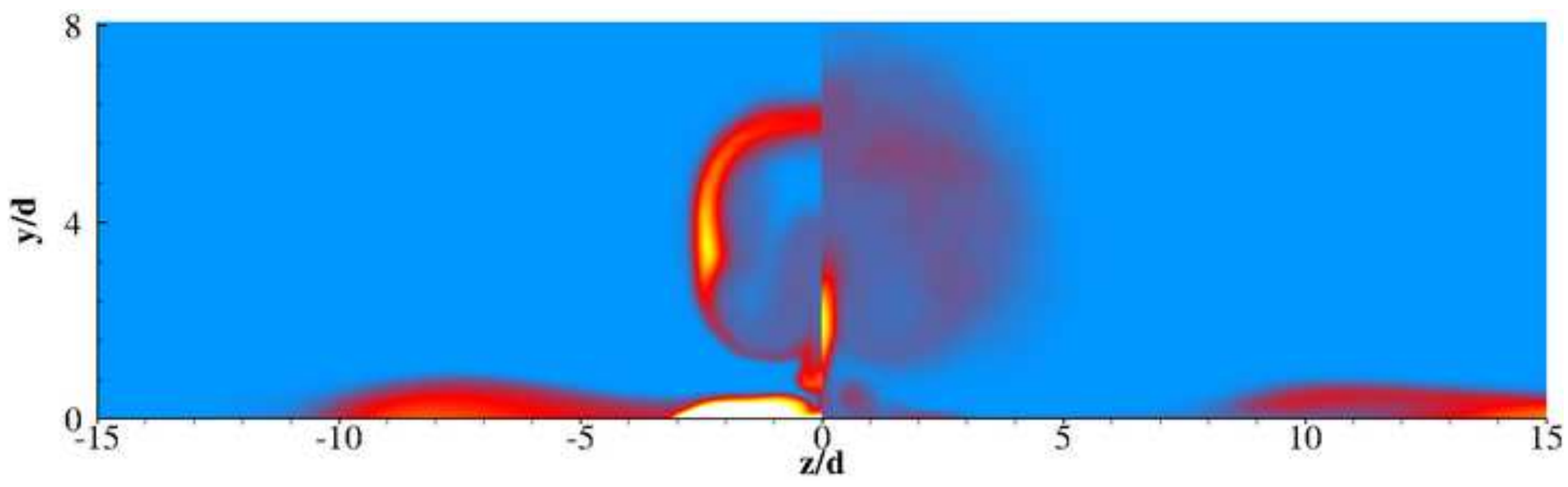


Figure $16 i$

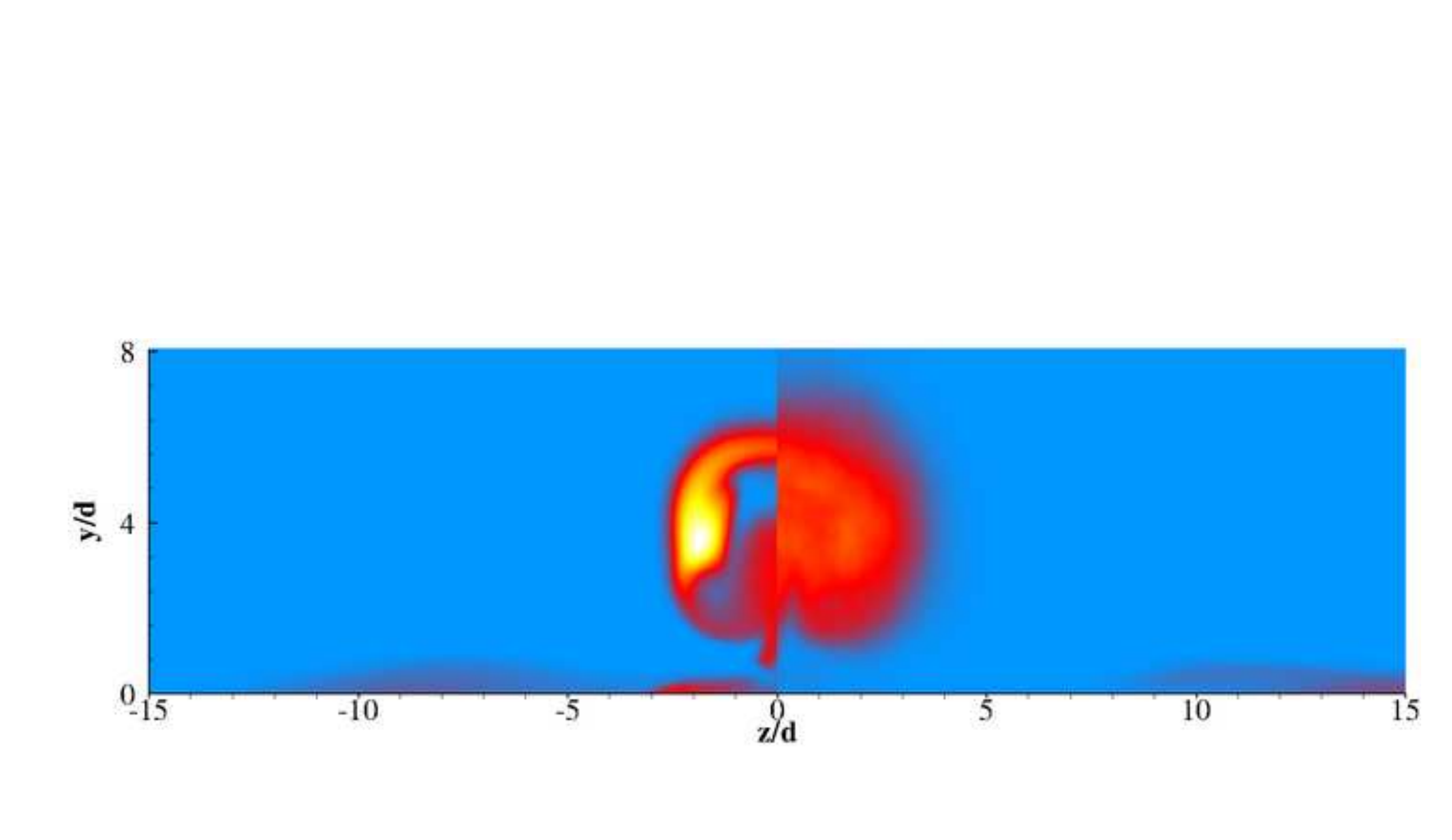
(
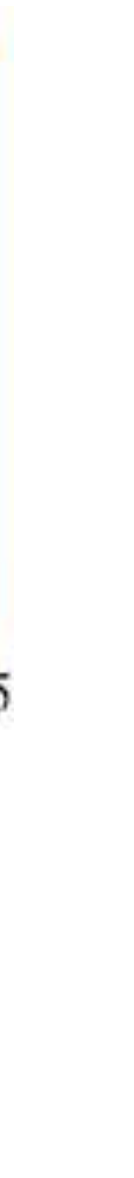


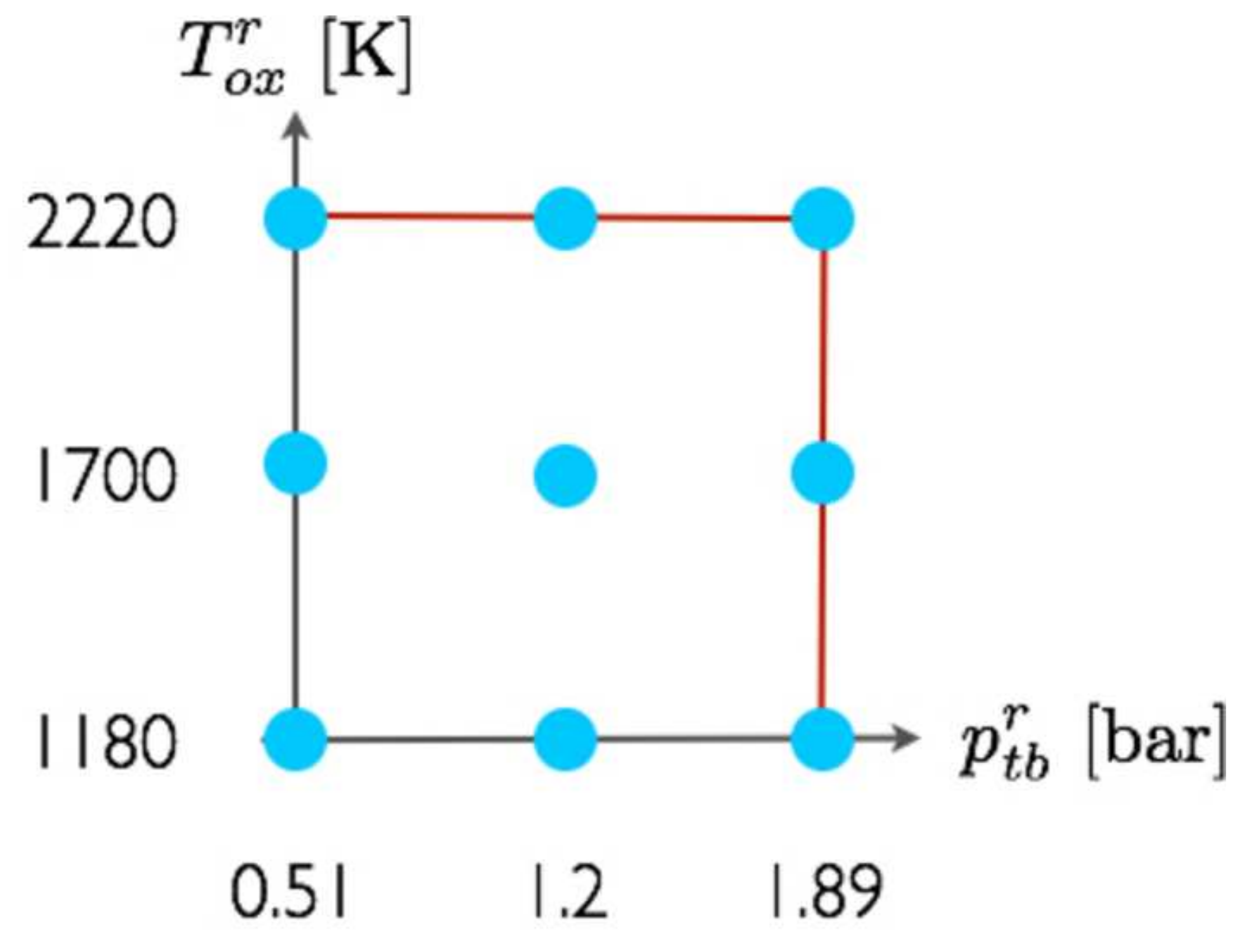

Figure 17
\[ \prod_{O X}^{T}[\mathbf{k}] \]

2220

1700

$\begin{array}{lll}0.5 & 1.2 & 1.89\end{array}$

$p_{t b}^{r}[\mathrm{bar}]$




Figure 18a

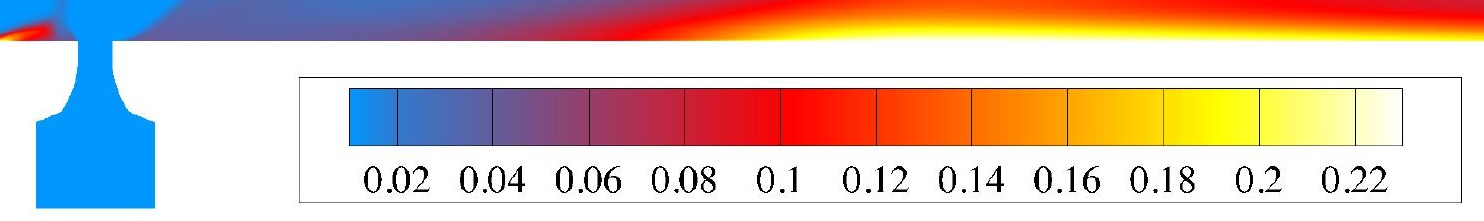

$$
\begin{array}{lllllllllll}
0.02 & 0.04 & 0.06 & 0.08 & 0.1 & 0.12 & 0.14 & 0.16 & 0.18 & 0.2 & 0.22
\end{array}
$$

(1) a 
Figure 20a

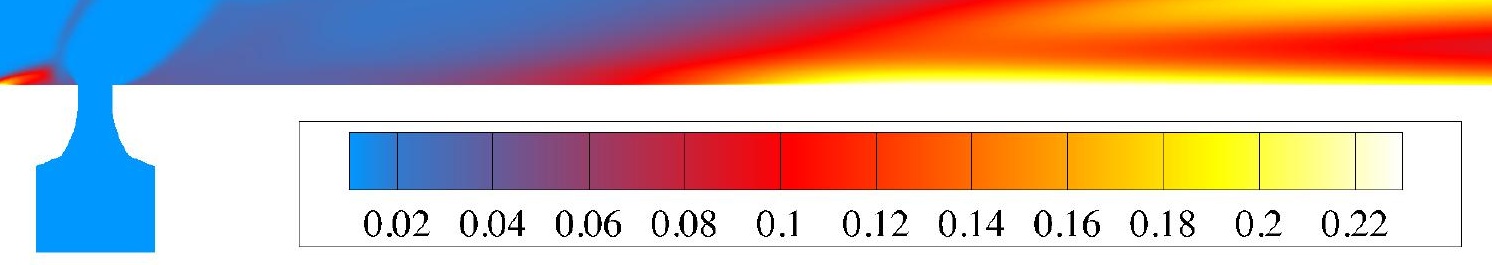

$$
\begin{array}{lllllllllll}
0.02 & 0.04 & 0.06 & 0.08 & 0.1 & 0.12 & 0.14 & 0.16 & 0.18 & 0.2 & 0.22
\end{array}
$$

\author{
Figure 20a
}

(1)

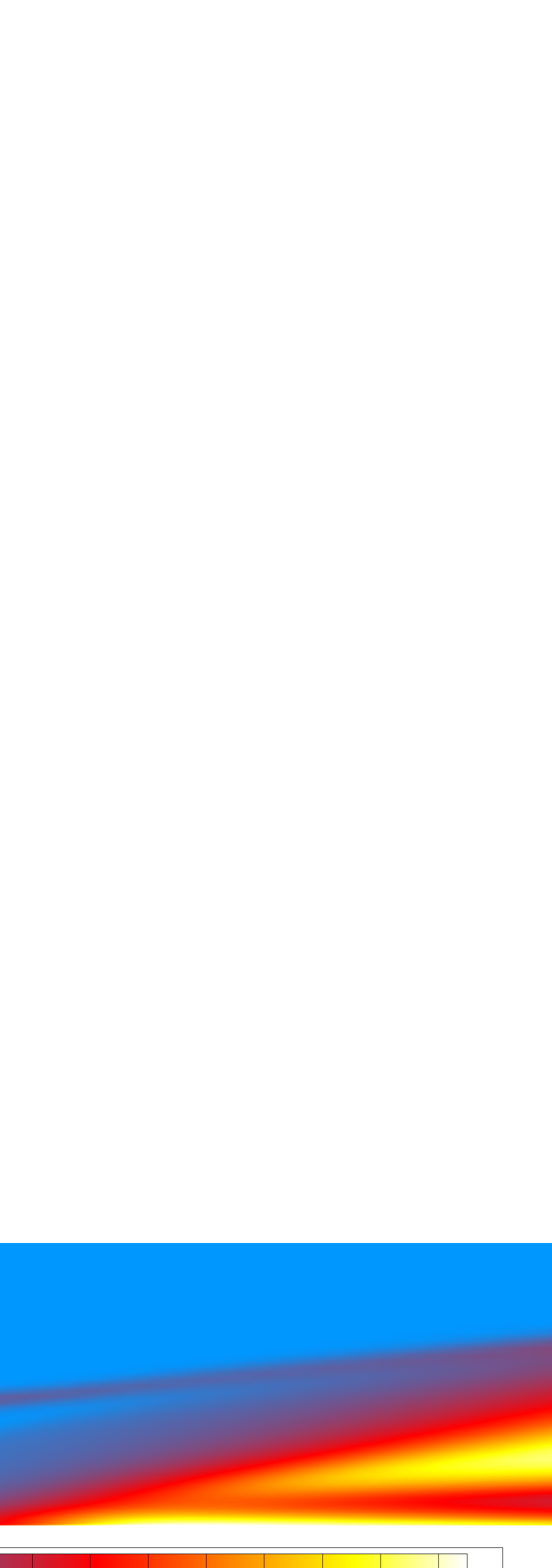


Figure $20 \mathrm{~b}$
Click here to download Figure: C_1180_noRescaling.eps



